JOURNAL OF THE AMERICAN MATHEMATICAL SOCIETY

Volume 21, Number 1, January 2008, Pages 171-234

S 0894-0347(07)00574-7

Article electronically published on June 25, 2007

\title{
PARABOLIC TRANSFER FOR REAL GROUPS
}

\author{
JAMES ARTHUR
}

\section{INTRODUCTION}

This paper is the second of two articles in real harmonic analysis. In the first paper A14, we established asymptotic formulas for some natural distributions on a real group. In this paper we shall establish important relationships among the distributions as the group varies.

The group is the set of real points of a connected reductive group $G$ over $\mathbb{R}$. The distributions are weighted orbital integrals $J_{M}(\gamma, f)$ on $G(\mathbb{R})$, and their invariant counterparts $I_{M}(\gamma, f)$. Here, $M \subset G$ is a Levi subgroup of $G$, while $\gamma \subset M_{G \text {-reg }}(\mathbb{R})$ is a strongly $G$-regular conjugacy class in $M(\mathbb{R})$. The relationships are defined by the invariant transfer of functions on $G(\mathbb{R})$ to functions attached to endoscopic groups of $G$. This necessitates our working with the invariant distributions $I_{M}(\gamma, f)$. We refer the reader to the introduction of [A14] for some general remarks on these objects.

The distributions $I_{M}(\gamma, f)$ are the generic archimedean terms in the invariant trace formula. We cannot review the trace formula here. The reader might consult the introductions to $\mathrm{A} 13$ and its two predecessors for a brief summary. The purpose of the paper A13] was to stabilize the invariant trace formula, subject to a condition on the fundamental lemma that has been established in some special cases. The stable trace formula is a milestone of sorts. It is expected to lead to reciprocity laws, which relate fundamental arithmetic data attached to automorphic representations on different groups.

The stable trace formula of [A13] relies upon the results of this paper (as well as a paper A16 in preparation). This has been our guiding motivation. The relevant identities among the nonarchimedean forms of the distributions $I_{M}(\gamma, f)$ were actually established in A13. They were a part of the global argument that culminated in the stable trace formula. As such, they are subject to the condition on the fundamental lemma mentioned above. Our goal here is to establish the outstanding archimedean identities. We shall do so by purely local means, which are independent of the fundamental lemma.

To simplify the Introducton, we assume that the derived group of $G$ is simply connected. The identities then relate the invariant distributions on $G(\mathbb{R})$ with stable distributions on endoscopic groups $G^{\prime}(\mathbb{R})$. We recall that a stable distribution on $G^{\prime}(\mathbb{R})$ depends only on the average values assumed by a test function over strongly regular stable conjugacy classes in $G_{\text {reg }}^{\prime}(\mathbb{R})$, which is to say, intersections of $G_{\text {reg }}^{\prime}(\mathbb{R})$

Received by the editors December 21, 2005.

2000 Mathematics Subject Classification. Primary 22E30, 22 E55.

The author was supported in part by NSERC Operating Grant A3483.

(C)2007 American Mathematical Society 
with conjugacy classes in $G^{\prime}(\mathbb{C})$. (An invariant distribution on $G(\mathbb{R})$ satisfies the broader condition of being invariant under conjugation by $G(\mathbb{R})$.) In general, the endoscopic groups represent a finite family $\left\{G^{\prime}\right\}$ of quasisplit groups over $\mathbb{R}$. They are defined in terms of $G$ by a purely algebraic construction [L1] of Langlands. For any $G^{\prime}$, Shelstad has established a correspondence $f \rightarrow f^{\prime}$ between test functions on $G(\mathbb{R})$ and $G^{\prime}(\mathbb{R})$. The image $f^{\prime}$ of $f$ is defined only up to its averages over stable conjugacy classes in $G^{\prime}(\mathbb{R})$, but this is enough to yield a pairing

$$
\left(f, S^{\prime}\right) \longrightarrow \widehat{S}^{\prime}\left(f^{\prime}\right)
$$

of $f$ with any stable distribution $S^{\prime}$ on $G^{\prime}(\mathbb{R})$. The identities express $I_{M}(\gamma, f)$ as a linear combination over $G^{\prime}$ of such pairings.

Let us be more precise. We fix an elliptic endoscopic datum $M^{\prime}$ for the Levi subgroup $M$ of $G$. We then attach an invariant distribution $I_{M}\left(\sigma^{\prime}, f\right)$ to any strongly $G$-regular element $\sigma^{\prime}$ in $M^{\prime}(\mathbb{R})$. This is a straightforward step, taken by applying the Shelstad correspondence for $M$ to the function

$$
\gamma \longrightarrow I_{M}(\gamma, f)
$$

The object is to relate this invariant distribution on $G(\mathbb{R})$ to stable distributions on endoscopic groups $G^{\prime}$ for $G$. The identity we eventually establish is a decomposition

$$
I_{M}\left(\sigma^{\prime}, f\right)=\sum_{G^{\prime}} \iota_{M^{\prime}}\left(G, G^{\prime}\right) \widehat{S}_{M^{\prime}}^{G^{\prime}}\left(\sigma^{\prime}, f^{\prime}\right)
$$

of $I_{M}\left(\sigma^{\prime}, f\right)$ into a finite sum, where $G^{\prime}$ ranges over endoscopic groups for which $M^{\prime}$ is a Levi subgroup, $\iota_{M^{\prime}}\left(G, G^{\prime}\right)$ are explicit coefficients, and $S_{M^{\prime}}^{G^{\prime}}\left(\sigma^{\prime}, \cdot\right)$ are uniquely determined stable distributions on the groups $G^{\prime}(\mathbb{R})$. This result can be regarded as a stabilization of the invariant distribution $I_{M}(\gamma, f)$.

The identity $(*)$ might seem somewhat arcane, at least at first reading. However, it is quite natural. It is governed by the very considerations whose global expression in A13 led to the stable trace formula. The essential point may be summarized as follows. One can actually try to stabilize $I_{M}(\gamma, f)$ in either of the two arguments $\gamma$ or $f$. However, it is not a priori evident that the two operations are compatible. Indeed, $I_{M}(\gamma, f)$ is defined as an invariant distribution by a rather formal process A14, (1.4)], which gives no indication of how the values it takes on averages of $f$ over conjugacy classes depend on $\gamma$. The two sides of the identity $(*)$, defined precisely in $\S 1$, represent the two ways of stabilizing $I_{M}(\gamma, f)$. The identity itself can thus be regarded as an assertion that the two operations are indeed compatible.

How can one establish an identity $(*)$ with so little knowledge of the explicit behaviour of $I_{M}(\gamma, f)$ ? The answer comes from an interesting application of methods of classical analysis. One shows that any of the terms in $(*)$ is the solution of a (nonhomogeneous) linear boundary value problem. Namely, it satisfies a system of linear differential equations, it obeys explicit boundary conditions as $\sigma^{\prime}$ approaches the $G$-singular set in $M^{\prime}(\mathbb{R})$, and it has an explicit asymptotic formula as $\sigma^{\prime}$ approaches infinity in $M^{\prime}(\mathbb{R})$.

We formulate the identity, in precise and somewhat more general terms, as Theorem 1.1 at the end of $\S 1$. The rest of the paper will be devoted to its proof.

The titles of the various sections are self-explanatory. We discuss the differential equations in $\S 2$. This is partly a review of the paper A12, where it was shown that the differential equations obeyed by $I_{M}(\gamma, f)$ satisfy infinitesimal analogues of the identity $(*)$ we are trying to establish. In $\S 3$, we investigate the boundary conditions 
attached to compact roots. This reduces to results of Shelstad [S1] for invariant orbital integrals, which were a part of her construction of the transfer mapping $f \rightarrow f^{\prime}$. In $\S 4$, we investigate the boundary conditions attached to noncompact roots. These results require more effort, since they have no analogue for invariant orbital integrals. We then analyze the asymptotic formula of A14 in $\S 5$. The problem is to convert the asymptotic expression for $I_{M}(\gamma, f)$ into a corresponding formula for the term $I_{M}\left(\sigma^{\prime}, f\right)$ on the left hand side of $(*)$, and compatible formulas for the terms $\widehat{S}_{\tilde{M}^{\prime}}^{\tilde{G}^{\prime}}\left(\sigma^{\prime}, f^{\prime}\right)$ on the right. This requires a separate stabilization of each side of the original asymptotic formula. In the last section $\S 6$, we combine everything. We show that the difference between the two sides of $(*)$ is the unique solution of a homogeneous boundary value problem, and hence vanishes.

Our results are obviously dependent on the work of Shelstad on real groups. Her construction of the mapping $f \rightarrow f^{\prime}$ was by geometric transfer, in terms of invariant orbital integrals. She later showed that the mapping could also be defined by a compatible spectral transfer $\mathbf{S 3}$, given by $L$-packets of tempered representations. We shall need both interpretations. Shelstad based her construction on ad hoc transfer factors, which predated (and anticipated) the systematic transfer factors of [LS1. This circumstance makes it difficult at times to keep track of her arguments. With the hindsight of [LS2, Theorem 2.6.A], we know that the mapping $f \rightarrow f^{\prime}$ can also be defined by means of the general transfer factors of [LS1. It would be very useful to reformulate Shelstad's arguments in terms of the general constructions of LS1. Rather than attempting to do so here, however, we have simply appealed to the original arguments whenever necessary.

The distributions $I_{M}(\gamma, f)$ that are the source of our identities are subtle objects. It is perhaps surprising that one can solve the problems implicit in $(*)$ by purely local methods. They could probably have been handled more easily by global means, as was done for the nonarchimedean valuations in A13. This is in fact the way the archimedean valuations were treated in the special case established in AC. However, we would still have needed all the local results established in $\S 2-4$ of this paper. Moreover, the final result would then have been conditional upon the generalized fundamental lemma on which A13 is predicated.

There are other reasons for proving as much as possible by local means. Langlands has recently outlined a tentative strategy [L5] for applying the trace formula much more broadly. While it has yet to be seen to work, even in principle, the strategy offers the possibility of something that has always been missing: a systematic attack on the general principle of functoriality. The next step is by no means clear. However, the theory of endoscopy has been instructive. Shelstad's study of invariant archimedean orbital integrals led to the general transfer factors needed for a precise theory of endoscopic transfer at any place. One can hope that the program outlined in [5], though much more difficult, will ultimately turn out to have structure in common with the theory of endoscopy. If this is so, a careful study of the archimedean terms in the stable trace formula would offer guidance. It could yield theorems required along the way, suggest what needs to be established at nonarchimedean places, and at the very least, provide evidence in support of the program. Some analysis of this sort has been carried out by Langlands [L4] for weighted orbital integrals on the group $G L(2, \mathbb{R})$. 


\section{$\S 1$. STATEMENT OF THE THEOREM}

We shall work in a slightly different context than is usual for real groups. We take $G$ to be a $K$-group over the field $\mathbb{R}$. The notion of a $K$-group was introduced in [A11. It is an algebraic variety

$$
G=\coprod_{\iota \in \pi_{0}(G)} G_{\iota}
$$

over $\mathbb{R}$, whose connected components $G_{\iota}$ are connected reductive groups over $\mathbb{R}$, and which is equipped with some extra structure.

The supplementary structure is an equivalence class of frames $(\psi, u)$ that also satisfies the cohomological condition at the beginning of $\S 2$ of A11. We recall that a frame is a family of pairs

$$
(\psi, u)=\left\{\left(\psi_{\iota \kappa}, u_{\iota \kappa}\right): \iota, \kappa \in \pi_{0}(G)\right\}
$$

where $\psi_{\iota}: G_{\kappa} \rightarrow G_{\iota}$ is an isomorphism of connected groups over $\mathbb{C}$, and $u_{\iota \kappa}$ is a function from the Galois group $\Gamma=\Gamma_{\mathbb{R}}=\operatorname{Gal}(\mathbb{C} / \mathbb{R})$ to the simply connected cover $G_{\iota, \mathrm{sc}}$ of the derived group $G_{\iota, \mathrm{der}}$ of $G$. The objects $\left\{\psi_{\iota \kappa}\right\}$ and $\left\{u_{\iota \kappa}\right\}$ are required to have the three properties of compatibility listed at the beginning of $\S 1$ of [A11. The cohomological condition is a further requirement, which includes the stipulation that each function $u_{\iota \kappa}$ be a one-cocycle. Moreover, for any fixed $\iota$, the mapping that sends $u_{\iota \kappa}$ to its image in $H^{1}\left(\mathbb{R}, G_{\iota}\right)$ is required to be a bijection from the set $\left\{u_{\iota \kappa}: \kappa \in \pi_{0}(G)\right\}$ onto the image of $H^{1}\left(\mathbb{R}, G_{\iota, \mathrm{sc}}\right)$ in $H^{1}\left(\mathbb{R}, G_{\iota}\right)$.

We assume a familiarity with the discussion of the first few sections of A11. Among other things, this includes the notion of a Levi $(K-)$ subgroup of $G$. Any Levi $(K-)$ subgroup $M$ comes with associated finite sets $\mathcal{P}(M), \mathcal{L}(M)$, and $\mathcal{F}(M)$, which play the same role as in the connected case. We can also form a dual group $\widehat{G}$ for $G$, and a dual Levi subgroup $\widehat{M} \subset \widehat{G}$ for $M$. Any such $\widehat{M}$ comes with a bijection $L \rightarrow \widehat{L}$ from $\mathcal{L}(M)$ to $\mathcal{L}(\widehat{M})$, and a bijection $P \rightarrow \widehat{P}$ from $\mathcal{P}(M)$ to $\mathcal{P}(\widehat{M})$. We recall that $\mathcal{P}(\widehat{M}), \mathcal{L}(\widehat{M})$, and $\mathcal{F}(\widehat{M})$ each consist of subgroups of $\widehat{G}$ that are stable under the action of $\Gamma$.

The definition of a $K$-group is clearly somewhat artificial. It was introduced only to streamline some aspects of the theory of endoscopy for connected groups. The main theorem of this paper could well be stated in terms of connected groups. However, the statement for $K$-groups we shall give presently is somewhat stronger.

Invariant harmonic analysis for connected real groups extends in a natural way to $K$-groups. As in [A11, we make use of obvious extensions to $G$ of standard notation for connected groups. For example, we have the Schwartz space

$$
\mathcal{C}(G)=\bigoplus_{\iota \in \pi_{0}(G)} \mathcal{C}\left(G_{\iota}\right)
$$

on $G(\mathbb{R})$ and its invariant analogue

$$
\mathcal{I}(G)=\bigoplus_{\iota \in \pi_{0}(G)} \mathcal{I}\left(G_{\iota}\right)
$$

Elements in $\mathcal{C}(G)$ are functions on $G(\mathbb{R})$. Elements in $\mathcal{I}(G)$ can be regarded either as functions on the disjoint union

$$
\Pi_{\text {temp }}(G)=\coprod_{\iota} \Pi_{\text {temp }}\left(G_{\iota}\right)
$$


of sets of irreducible tempered representations on the groups $G_{\iota}(\mathbb{R})$ or as functions on the disjoint union

$$
\Gamma_{\mathrm{reg}}(G)=\coprod_{\iota} \Gamma_{\mathrm{reg}}\left(G_{\iota}\right)
$$

of sets of strongly regular conjugacy classes in the groups $G_{\iota}(\mathbb{R})$.

For purposes of induction, it is convenient to fix a central character datum $(Z, \zeta)$ for $G$. Then $Z$ is an induced torus over $\mathbb{R}$, with central embeddings

$$
Z \stackrel{\sim}{\longrightarrow} Z_{\iota} \subset G_{\iota}
$$

that are compatible with the isomorphisms $\psi_{\iota \kappa}$. (Recall that an induced torus over a field $F$ is a product of tori of the form $R_{E / F}(G L(1))$.) The second component $\zeta$ is a character on $Z(\mathbb{R})$, which transfers to a character $\zeta_{\iota}$ on $Z_{\iota}(\mathbb{R})$ for each $\iota$. We can then form the space

$$
\mathcal{C}(G, \zeta)=\bigoplus_{\iota} \mathcal{C}\left(G_{\iota}, \zeta_{\iota}\right)
$$

of $\zeta^{-1}$-equivariant Schwartz functions on $G(\mathbb{R})$ and its invariant analogue

$$
\mathcal{I}(G, \zeta)=\bigoplus_{\iota} \mathcal{I}\left(G_{\iota}, \zeta_{\iota}\right)
$$

Elements in $\mathcal{I}(G, \zeta)$ may be regarded either as $\zeta^{-1}$-equivariant functions on $\Gamma_{\text {reg }}(G)$, or as functions on the set

$$
\Pi_{\text {temp }}(G, \zeta)=\coprod_{\iota} \Pi_{\text {temp }}\left(G_{\iota}, \zeta_{\iota}\right)
$$

of representations in $\Pi_{\text {temp }}(G)$ whose $Z(\mathbb{R})$-central character equals $\zeta$.

The paper [14] of which this is a continuation was written for connected groups with trivial central character datum. The definitions and constructions of A14 extend easily to the $K$-group $G$ with arbitrary central character datum $(Z, \zeta)$. We adopt them here, often without further comment. In particular, we form the invariant tempered distributions

$$
I_{M}(\gamma, f)=I_{M}^{G}(\gamma, f), \quad f \in \mathcal{C}(G, \zeta),
$$

indexed by Levi $K$-subgroups $M$ of $G$ and strongly $G$-regular elements $\gamma \in M(\mathbb{R})$. In the present setting, $I_{M}(\gamma, f)$ is a $\zeta$-equivariant distribution in $f$ and a $\zeta^{-1}$. equivariant function of $\gamma$. In the special case that $M=G$, the distribution

$$
f_{G}(\gamma)=I_{G}(\gamma, f)
$$

is essentially Harish-Chandra's invariant orbital integral. We recall that the invariant function space above is defined as the family

$$
\mathcal{I}(G, \zeta)=\left\{f_{G}: f \in \mathcal{C}(G, \zeta)\right\},
$$

regarded as a space of functions of $\gamma \in \Gamma_{\text {reg }}(G)$. However, it is the case of general $M$ that is of interest here. Our aim is to study the stabilization of the general distributions $I_{M}(\gamma, f)$.

There are two ways one could try to stabilize $I_{M}(\gamma, f)$, corresponding to the two arguments $\gamma$ and $f$. If $M=G$, the two stabilizations are the same. In this case, Langlands and Shelstad use the transfer factors of [LS1, and the resulting stabilization in $\gamma$, as the definition of the transfer mapping $f \rightarrow f^{\prime}$ that stabilizes $f$. If $M \neq G$, the two possible ways of stabilizing $I_{M}(\gamma, f)$ are thus predetermined. 
They are dictated by the constructions from the more elementary case that $M=G$. The purpose of this paper, simply put, is to show that they are compatible.

We are assuming a knowledge of the basic constructions of A11. These include the extension to $K$-groups [A11, §2] of the transfer factors of Langlands and Shelstad, a construction that follows observations of Vogan and Kottwitz. However, we shall also view matters from a slightly broader perspective. This is because some of the constructions become more natural if we treat all the transfer factors attached to a given endoscopic datum at the same time.

Suppose that $G^{\prime}$ represents an endoscopic datum $\left(G^{\prime}, \mathcal{G}^{\prime}, s^{\prime}, \xi^{\prime}\right)$ for $G$ [LS1, (1.2)]. In particular, $G^{\prime}$ is a connected quasisplit group over $\mathbb{R}$. A transfer factor for $G$ and $G^{\prime}$ includes an implicit choice of auxiliary datum $\left(\widetilde{G}^{\prime}, \widetilde{\xi}^{\prime}\right)$, in which $\widetilde{G}^{\prime}$ is an $\mathbb{R}$-rational central extension of $G^{\prime}$ by an induced torus $\widetilde{C}^{\prime}$, and $\widetilde{\xi}^{\prime}$ is an admissible $L$-embedding of $\mathcal{G}^{\prime}$ into ${ }^{L} \widetilde{G}^{\prime}$. For example, one could take $\widetilde{G}^{\prime}$ to be a $z$-extension of $G^{\prime}$ [K1]. The derived group of $\widetilde{G}^{\prime}$ is then simply connected, and an embedding $\widetilde{\xi}^{\prime}$ can always be found [L1]. A transfer factor attached to $\left(\widetilde{G}^{\prime}, \widetilde{\xi}^{\prime}\right)$ is a function

$$
\Delta_{G}\left(\delta^{\prime}, \gamma\right), \quad \delta^{\prime} \in \Delta_{G-\mathrm{reg}}\left(\widetilde{G}^{\prime}\right), \gamma \in \Gamma_{\mathrm{reg}}(G),
$$

which vanishes unless the projection of $\delta^{\prime}$ onto $G^{\prime}(\mathbb{R})$ is an image [LS1, (1.3)] of $\gamma$. Its purpose is to transfer functions $f \in \mathcal{C}(G, \zeta)$ to functions

$$
f^{\prime}\left(\delta^{\prime}\right)=f^{\tilde{G}^{\prime}}\left(\delta^{\prime}\right)=\sum_{\gamma \in \Gamma_{\mathrm{reg}}(G)} \Delta_{G}\left(\delta^{\prime}, \gamma\right) f_{G}(\gamma)
$$

of $\delta^{\prime}$ in the set $\Delta_{G \text {-reg }}\left(\widetilde{G}^{\prime}\right)$ of strongly $G$-regular stable conjugacy classes in $\widetilde{G}^{\prime}(\mathbb{R})$. We write $\widetilde{Z}^{\prime}$ for the extension of $Z$ by $\widetilde{C}^{\prime}$ given by the preimage of $Z$ in $\widetilde{G}^{\prime}, \widetilde{\eta}^{\prime}$ for the character on $\widetilde{Z}^{\prime}(\mathbb{R})$ determined by the auxiliary datum, and $\widetilde{\zeta}^{\prime}$ for the product of $\widetilde{\eta}^{\prime}$ with (the pullback of) $\zeta$. Then

$$
f^{\prime}\left(z^{\prime} \delta^{\prime}\right)=\widetilde{\zeta}^{\prime}\left(z^{\prime}\right)^{-1} f^{\prime}\left(\delta^{\prime}\right), \quad z^{\prime} \in \widetilde{Z}^{\prime}(\mathbb{R}) .
$$

(See [A11, §2], where the objects $\widetilde{C}^{\prime}, \widetilde{Z}^{\prime}, \widetilde{\eta}^{\prime}$ and $\widetilde{\zeta}^{\prime}$ were denoted by $\widetilde{Z}^{\prime}, \widetilde{Z}^{\prime} Z, \widetilde{\zeta}^{\prime}$ and $\widetilde{\zeta}^{\prime} \zeta$, respectively.) It follows from results of Shelstad [S3] that $f^{\prime}$ belongs to the space

$$
\mathcal{S}\left(\widetilde{G}^{\prime}, \widetilde{\zeta}^{\prime}\right)=\left\{h^{\tilde{G}^{\prime}}: h \in \mathcal{C}\left(\widetilde{G}^{\prime}, \widetilde{\zeta}^{\prime}\right)\right\}
$$

of stable orbital integrals on $\widetilde{G}^{\prime}(\mathbb{R})$.

What ambiguity is there in the choice of a transfer factor? If $\left(\widetilde{G}^{\prime}, \widetilde{\xi}^{\prime}\right)$ is fixed, $\Delta=\Delta_{G}$ can be replaced by a scalar multiple

$$
(u \Delta)\left(\delta^{\prime}, \gamma\right)=u \Delta\left(\delta^{\prime}, \gamma\right)
$$

by a complex number $u \in U(1)$ of absolute value 1 , but is otherwise uniquely determined. If only $\widetilde{G}^{\prime}$ is fixed, $\widetilde{\xi}^{\prime}$ can be replaced by a multiple $\alpha^{\prime} \widetilde{\xi}^{\prime}$, where $\alpha^{\prime}$ is a 1-cocycle from the real Weil group $W_{\mathbb{R}}$ into the center $Z\left(\widehat{\widetilde{G}^{\prime}}\right)$ of $\widehat{\widetilde{G}}^{\prime}$. The Langlands correspondence for tori, combined with the constructions of [LS1] (especially (3.5) and (4.4)), tells us that there is a canonical character $\omega^{\prime}$ on $\widetilde{G}^{\prime}(\mathbb{R})$ such that the product

$$
\left(\omega^{\prime} \Delta\right)\left(\delta^{\prime}, \gamma\right)=\omega^{\prime}\left(\delta^{\prime}\right) \Delta\left(\delta^{\prime}, \gamma\right)
$$

is a transfer factor attached to $\left(\widetilde{G}^{\prime}, \alpha^{\prime} \widetilde{\xi}^{\prime}\right)$. (We assume implicitly that our admissible embeddings are of unitary type, in the sense that they have bounded image in the 
abelian quotient $\widehat{\widetilde{G}}_{\text {ab }}^{\prime}$ of $\widehat{\widetilde{G}}^{\prime}$. This forces $\omega^{\prime}$ to be unitary.) Finally, we can replace $\widetilde{G}^{\prime}$ by some other central extension $\widetilde{G}_{1}^{\prime}$ of $G^{\prime}$. By taking fibre products, one sees that it is enough to consider the case that $\widetilde{G}_{1}^{\prime}$ is a central extension of $\widetilde{G}^{\prime}$. The composition $\widetilde{\xi}_{1}^{\prime}$ of a given $\widetilde{\xi}^{\prime}$ with the standard $L$-embedding of ${ }^{L} \widetilde{G}^{\prime}$ into ${ }^{L} \widetilde{G}_{1}^{\prime}$ is then an admissible embedding of $\mathcal{G}^{\prime}$ into ${ }^{L} \widetilde{G}_{1}^{\prime}$. In this case, the function

$$
\Delta_{1}\left(\delta_{1}^{\prime}, \gamma\right)=\Delta\left(\delta^{\prime}, \gamma\right), \quad \delta_{1}^{\prime} \in \Delta_{G-\operatorname{reg}}\left(\widetilde{G}_{1}^{\prime}\right),
$$

in which $\delta^{\prime}$ is the image of $\delta_{1}^{\prime}$ in $\widetilde{G}^{\prime}(F)$, is a transfer factor for $\left(\widetilde{G}_{1}^{\prime}, \widetilde{\xi}_{1}^{\prime}\right)$.

It is obvious how the Langlands-Shelstad transfer mapping $f \rightarrow f^{\prime}=f_{\Delta}^{\prime}$ depends on $\Delta$. The definitions immediately lead to the three relations

$$
\left\{\begin{array}{l}
f_{u \Delta}^{\prime}\left(\delta^{\prime}\right)=u f_{\Delta}^{\prime}\left(\delta^{\prime}\right), \\
f_{\omega^{\prime} \Delta}^{\prime}\left(\delta^{\prime}\right)=\omega^{\prime}\left(\delta^{\prime}\right) f_{\Delta}^{\prime}\left(\delta^{\prime}\right), \\
f_{\Delta_{1}}^{\prime}\left(\delta_{1}^{\prime}\right)=f_{\Delta}^{\prime}\left(\delta^{\prime}\right),
\end{array}\right.
$$

governed by the three objects $u, \omega^{\prime}$ and $\Delta_{1}$ above. These relations, which will be an implicit part of our understanding, have obvious analogues for the Levi subgroup $M$.

Suppose that $M^{\prime}$ represents a fixed endoscopic datum $\left(M^{\prime}, \mathcal{M}^{\prime}, s_{M}^{\prime}, \xi_{M}^{\prime}\right)$ for $M$. Let us write $\mathcal{T}\left(M, M^{\prime}\right)$ for the corresponding set of transfer factors for $M$ and $M^{\prime}$. An element $\Delta_{M} \in \mathcal{T}\left(M, M^{\prime}\right)$ thus comes with an underlying auxiliary datum $\left(\widetilde{M}^{\prime}, \widetilde{\xi}_{M}^{\prime}\right)$, and a character $\widetilde{\zeta}^{\prime}$ on the central subgroup $\widetilde{Z}^{\prime}(\mathbb{R})$ of $\widetilde{M}^{\prime}(\mathbb{R})$ that depends on the original character $\zeta$ on $Z(\mathbb{R})$. To make matters more concrete in the present setting of real groups, we fix a maximal torus $T^{\prime}$ in $M^{\prime}$ over $\mathbb{R}$, with preimage $\widetilde{T}^{\prime}$ in $\widetilde{M}^{\prime}$. We will then work with points $\sigma^{\prime} \in \widetilde{T}_{G \text {-reg }}^{\prime}(\mathbb{R})$, instead of the stable conjugacy classes $\delta^{\prime}$ in $\widetilde{M}^{\prime}(\mathbb{R})$ they represent. (As usual, the subscript $G$-reg denotes the subset of elements in a given set that are $G$-regular.) We are going to treat families of suitably related functions, parametrized by elements $\Delta=\Delta_{M}$ in $\mathcal{T}\left(M, M^{\prime}\right)$ and defined on the associated spaces $\widetilde{T}_{G \text {-reg }}^{\prime}(\mathbb{R})$, as sections of an underlying line bundle.

We first introduce a bundle $\mathcal{L}\left(T^{\prime}, M, \zeta\right)$ of equivalence classes of pairs

$$
\left(\Delta, \sigma^{\prime}\right), \quad \Delta \in \mathcal{T}\left(M, M^{\prime}\right), \sigma^{\prime} \in \widetilde{T}^{\prime}(\mathbb{R}) .
$$

The equivalence relation is generated by the elementary relations

$$
\left(\Delta, z^{\prime} \sigma^{\prime}\right) \sim\left(\widetilde{\zeta}^{\prime}\left(z^{\prime}\right)^{-1} \Delta, \sigma^{\prime}\right), \quad z^{\prime} \in \widetilde{Z}^{\prime}(\mathbb{R}),
$$

and

$$
\left\{\begin{array}{l}
\left(\omega^{\prime} \Delta, \sigma^{\prime}\right) \sim\left(\omega^{\prime}\left(\sigma^{\prime}\right) \Delta, \sigma^{\prime}\right) \\
\left(\Delta_{1}, \sigma_{1}^{\prime}\right) \sim\left(\Delta, \sigma^{\prime}\right)
\end{array}\right.
$$

where $\omega^{\prime}$ is an arbitrary character on $\tilde{M}^{\prime}(\mathbb{R})$, and $\sigma_{1}^{\prime} \rightarrow \sigma^{\prime}$ and $\Delta \rightarrow \Delta_{1}$ are the mappings attached to a central extension $\widetilde{M}_{1}^{\prime} \rightarrow \widetilde{M}^{\prime}$, as above. The natural projection

$$
\left(\Delta, \sigma^{\prime}\right) \longrightarrow \bar{\sigma}^{\prime}
$$

makes $\mathcal{L}\left(T^{\prime}, M, \zeta\right)$ into a principal $U(1)$-bundle over the quotient

$$
\bar{T}^{\prime}(\mathbb{R})=\widetilde{T}^{\prime}(\mathbb{R}) / \widetilde{Z}^{\prime}(\mathbb{R})=T^{\prime}(\mathbb{R}) / Z(\mathbb{R}) .
$$


We may as well write $\mathcal{L}\left(T^{\prime}, M, \zeta\right)$ also for the complex line bundle attached to the canonical one-dimensional representation of $U(1)$. We then form the dual line bundle $\mathcal{L}^{*}\left(T^{\prime}, M, \zeta\right)$, and its restriction $\mathcal{L}^{*}\left(T_{G \text {-reg }}^{\prime}, M, \zeta\right)$ to $\bar{T}_{G \text {-reg }}^{\prime}(\mathbb{R})$.

With these definitions, we write $C^{\infty}\left(T_{G \text {-reg }}^{\prime}, M, \zeta\right)$ for the space of smooth sections of the bundle $\mathcal{L}^{*}\left(T_{G \text {-reg }}^{\prime}, M, \zeta\right)$. An element in this space is thus a complex valued function

$$
a^{\prime}:\left(\Delta, \sigma^{\prime}\right) \longrightarrow a_{\Delta}^{\prime}\left(\sigma^{\prime}\right), \quad \Delta \in \mathcal{T}\left(M, M^{\prime}\right), \sigma^{\prime} \in \widetilde{T}_{G-\mathrm{reg}}^{\prime}(\mathbb{R}),
$$

that satisfies relations

$$
a_{\Delta}^{\prime}\left(z^{\prime} \sigma^{\prime}\right)=\widetilde{\zeta}^{\prime}\left(z^{\prime}\right)^{-1} a_{\Delta}^{\prime}\left(\sigma^{\prime}\right), \quad z^{\prime} \in \widetilde{Z}^{\prime}(\mathbb{R}),
$$

and

$$
\left\{\begin{array}{l}
a_{u \Delta}^{\prime}\left(\sigma^{\prime}\right)=u a_{\Delta}^{\prime}\left(\sigma^{\prime}\right), \\
a_{\omega^{\prime} \Delta}^{\prime}\left(\sigma^{\prime}\right)=\omega^{\prime}\left(\sigma^{\prime}\right) a_{\Delta}^{\prime}\left(\sigma^{\prime}\right), \\
a_{\Delta_{1}}^{\prime}\left(\sigma_{1}^{\prime}\right)=a_{\Delta}^{\prime}\left(\sigma^{\prime}\right),
\end{array}\right.
$$

parallel to (1.2) and (1.3). We need only specify the values taken by the function at one transfer factor $\Delta=\Delta_{M}$. We shall often do so, without including $\Delta_{M}$ explicitly in the notation. In other words, we shall write the section $a^{\prime}$ as a function of $\sigma^{\prime}$, rather than the point $\bar{\sigma}^{\prime}$ in the base space.

We assume from now on that the endoscopic datum $M^{\prime}$ for $M$ is elliptic. The first stabilization of $I_{M}(\gamma, f)$ is the more elementary. It simply transforms $I_{M}(\gamma, f)$ to the function

$$
I_{M}\left(\sigma^{\prime}, f\right)=\sum_{\gamma \in \Gamma_{G^{- \text {reg }}}(M)} \Delta_{M}\left(\sigma^{\prime}, \gamma\right) I_{M}(\gamma, f)
$$

of $\sigma^{\prime} \in \widetilde{T}_{G-\text { reg }}^{\prime}(\mathbb{R})$ attached to a transfer factor $\Delta_{M} \in \mathcal{T}\left(M^{\prime}, M\right)$. It is clear that as $\Delta_{M}$ varies, this function satisfies the relations $(1.2)_{M}$ and $(1.3)_{M}$. It can therefore be regarded as a section in the space $C^{\infty}\left(T_{G \text {-reg }}^{\prime}, M, \zeta\right)$. We have excluded $\Delta_{M}$ from the notation $I_{M}\left(\sigma^{\prime}, f\right)$, as agreed, with the understanding that there is an implicit dependence on $\Delta_{M}$ governed by $(1.3)_{M}$.

To describe the second stabilization, it is well to recall some other notions from the early part of A11. Replacing $M^{\prime}$ by an isomorphic endoscopic datum, if necessary, we assume that $\mathcal{M}^{\prime}$ is an $L$-subgroup of ${ }^{L} M$ and that $\xi_{M}^{\prime}$ is the identity embedding. We then form the family $\mathcal{E}_{M^{\prime}}(G)$ of endoscopic data for $G$, as for example in [A11, §3]. Thus, $\mathcal{E}_{M^{\prime}}(G)$ consists of data $\left(G^{\prime}, \mathcal{G}^{\prime}, s^{\prime}, \xi^{\prime}\right)$, taken up to translation of $s^{\prime}$ by $Z(\widehat{G})^{\Gamma}$, in which $s^{\prime}$ lies in $s_{M}^{\prime} Z(\widehat{M})^{\Gamma}, \widehat{G}^{\prime}$ is the connected centralizer of $s^{\prime}$ in $\widehat{G}, \mathcal{G}^{\prime}$ equals $\mathcal{M}^{\prime} \widehat{G}^{\prime}$, and $\xi^{\prime}$ is the identity $L$-embedding of $\mathcal{G}^{\prime}$ into ${ }^{L} G$. For any datum in $\mathcal{E}_{M^{\prime}}(G)$ (which we continue to represent by its first component $G^{\prime}$ ), the dual group $\widehat{M}^{\prime}$ of $M^{\prime}$ comes with the structure of a Levi subgroup of $\widehat{G}^{\prime}$. We fix an embedding $M^{\prime} \subset G^{\prime}$ for which $\widehat{M}^{\prime} \subset \widehat{G}^{\prime}$ is a dual Levi subgroup.

The $K$-group $G$ is assumed implicitly to have been equipped with a quasisplit inner twist

$$
\psi=\left\{\psi_{\iota}: G_{\iota} \longrightarrow G^{*}, \iota \in \pi_{0}(G)\right\},
$$

where $G^{*}$ is a connected quasisplit group over $\mathbb{R}$ [A11, $\left.\S 1\right]$. We say that $G$ is quasisplit if one of its components $G_{\iota}$ is quasisplit. In this case, one can arrange that $\psi_{\iota}$ is an $\mathbb{R}$-rational isomorphism from $G_{\iota}$ to $G^{*}$. We can then identify the function $f^{G}$ on $\Delta_{G \text {-reg }}(G)$ given by the stable orbital integrals of any $f \in \mathcal{C}(G, \zeta)$ with the 
function $f^{*}=f^{G^{*}}$ in $\mathcal{S}\left(G^{*}, \zeta^{*}\right)$ given by stable transfer. (See [A11, pp. 226-227].) In general, the transfer factors were defined explicitly [LS1] in terms of $\psi$. However, if we identify the dual group $\widehat{G}^{*}$ with $\widehat{G}, \psi$ is uniquely determined up to a natural equivalence relation. For this reason, we will usually not have occasion to refer to $\psi$. Of course the group $G^{*}$ plays an independent role as the maximal endoscopic datum for $G$. It lies in $\mathcal{E}_{M^{\prime}}(G)$ if and only if $M^{\prime}$ is the maximal endoscopic datum for $M$, which is to say that it equals the quasisplit inner form $M^{*}$ of $M$, a group that can also be regarded as a Levi subgroup of $G^{*}$. In general, we set

$$
\mathcal{E}_{M^{\prime}}^{0}(G)= \begin{cases}\mathcal{E}_{M^{\prime}}(G)-\left\{G^{*}\right\}, & \text { if } G \text { is quasisplit, } \\ \mathcal{E}_{M^{\prime}}(G), & \text { otherwise. }\end{cases}
$$

If $G^{\prime}$ is an arbitrary element in $\mathcal{E}_{M^{\prime}}(G)$, we also set

$$
\iota_{M^{\prime}}\left(G, G^{\prime}\right)=\left|Z\left(\widehat{M}^{\prime}\right)^{\Gamma} / Z(\widehat{M})^{\Gamma}\right|\left|Z\left(\widehat{G}^{\prime}\right)^{\Gamma} / Z(\widehat{G})^{\Gamma}\right|^{-1} .
$$

The second stabilization of $I_{M}(\gamma, f)$ is an inductive construction. Suppose that $G^{\prime} \in \mathcal{E}_{M^{\prime}}^{0}(G)$, that $\widetilde{G}^{\prime}$ is a central extension of $G^{\prime}$ by an induced central torus $\widetilde{C}^{\prime}$ over $\mathbb{R}$, and that $\widetilde{\zeta}^{\prime}$ is a character on the pullback $\widetilde{Z}^{\prime}(\mathbb{R})$ of $Z(\mathbb{R})$ to $\widetilde{G}^{\prime}(\mathbb{R})$. The preimage $\widetilde{M}^{\prime}$ of $M^{\prime}$ in $\widetilde{G}^{\prime}$ is then a Levi subgroup, while the preimage $\widetilde{T}^{\prime}$ of $T^{\prime}$ is a maximal torus. We assume inductively that for every such $G^{\prime}, \widetilde{G}^{\prime}$ and $\widetilde{\zeta}^{\prime}$, we have defined a family

$$
S_{\tilde{M}^{\prime}}^{\tilde{G}^{\prime}}\left(\sigma^{\prime}, h^{\prime}\right), \quad \sigma^{\prime} \in \widetilde{T}_{G-\mathrm{reg}}^{\prime}(\mathbb{R}), h^{\prime} \in \mathcal{C}\left(\widetilde{G}^{\prime}, \widetilde{\zeta}^{\prime}\right),
$$

of tempered, stable, $\widetilde{\zeta}^{\prime}$-equivariant distributions on $\widetilde{G}^{\prime}(\mathbb{R})$, with

$$
S_{\tilde{M}^{\prime}}^{\tilde{G}^{\prime}}\left(z^{\prime} \sigma^{\prime}, h^{\prime}\right)=\widetilde{\zeta}^{\prime}\left(z^{\prime}\right)^{-1} S_{\tilde{M}^{\prime}}^{\tilde{G}^{\prime}}\left(\sigma^{\prime}, h^{\prime}\right), \quad z^{\prime} \in \widetilde{Z}^{\prime}(\mathbb{R}) .
$$

We assume also that the relations

$$
\left\{\begin{array}{l}
S_{\tilde{M}^{\prime}}^{\tilde{G}^{\prime}}\left(\sigma^{\prime}, \omega^{\prime} h^{\prime}\right)=\omega^{\prime}\left(\sigma^{\prime}\right) S_{\tilde{M}^{\prime}}^{\tilde{G}^{\prime}}\left(\sigma^{\prime}, h^{\prime}\right), \\
S_{\tilde{M}_{1}^{\prime}}^{\tilde{G}_{1}^{\prime}}\left(\sigma_{1}^{\prime}, h_{1}^{\prime}\right)=S_{\tilde{M}^{\prime}}^{\tilde{G}^{\prime}}\left(\sigma^{\prime}, h^{\prime}\right),
\end{array}\right.
$$

hold, for a character $\omega^{\prime}$ on $\widetilde{G}(\mathbb{R})$, a covering $\widetilde{G}_{1}^{\prime}$ of $\widetilde{G}^{\prime}$ as in (1.3), and pullbacks $\widetilde{\zeta}_{1}$ and $h_{1}^{\prime}$ of $\widetilde{\zeta}^{\prime}$ and $h^{\prime}$ to $\widetilde{G}_{1}^{\prime}$.

Suppose that $\Delta$ is a transfer factor attached to an element $G^{\prime} \in \mathcal{E}_{M^{\prime}}^{0}(G)$. Then $\Delta$ comes with an auxiliary datum $\left(\widetilde{G}^{\prime}, \widetilde{\xi}^{\prime}\right)$ and a character $\widetilde{\zeta}^{\prime}$ on $\widetilde{Z}^{\prime}(\mathbb{R})$. It therefore gives rise to a collection of stable distributions $S_{\tilde{M}^{\prime}}^{\tilde{G}^{\prime}}\left(\sigma^{\prime}, \cdot\right)$ on $\widetilde{G}^{\prime}(\mathbb{R})$, by hypothesis. It also provides the transfer mapping $f \rightarrow f^{\prime}=f_{\Delta}^{\prime}$ from $\mathcal{C}(G, \zeta)$ to $\mathcal{S}\left(\widetilde{G}^{\prime}, \widetilde{\zeta}^{\prime}\right)$. We thus obtain a family of $\zeta$-equivariant distributions

$$
\widehat{S}_{\tilde{M}^{\prime}}^{\tilde{G}^{\prime}}\left(\sigma^{\prime}, f^{\prime}\right), \quad f \in \mathcal{C}(G, \zeta), \sigma^{\prime} \in \widetilde{T}_{G-\mathrm{reg}}^{\prime}(\mathbb{R}),
$$

on $G(\mathbb{R})$. (We write $\widehat{S}^{\prime}$, as usual, for the transfer of a stable linear form $S^{\prime}$ on $\mathcal{C}\left(\widetilde{G}^{\prime}, \widetilde{\zeta}^{\prime}\right)$ to a linear form on $\mathcal{S}\left(\widetilde{G}^{\prime}, \widetilde{\zeta}^{\prime}\right)$.) Now there is a canonical restriction mapping from $\mathcal{T}\left(G, G^{\prime}\right)$ to $\mathcal{T}\left(M, M^{\prime}\right)$, which takes $\Delta$ to a transfer factor $\Delta_{M}$ for $M$ with auxiliary datum $\left(\widetilde{M}^{\prime}, \widetilde{\xi}_{M}^{\prime}\right)$. It follows easily from (1.3), (1.5) and (1.6) that as a function of $\Delta_{M}$ and $\sigma^{\prime},(1.7)$ satisfies the relations $(1.3)_{M}$ (with $\Delta_{M}$ in place of $\Delta)$. In other words, (1.7) varies in the appropriate way as $\Delta_{M}$ ranges over the image of the injective mapping $\Delta \rightarrow \Delta_{M}$. It therefore extends to a section in $C^{\infty}\left(T_{G-\mathrm{reg}}^{\prime}, M, \zeta\right)$ 
We now recall the stabilization of $I_{M}(\gamma, f)$ with respect to the function $f$ in $\mathcal{C}(G, \zeta)$. If $G$ is not quasisplit, we define an "endoscopic" distribution

$$
I_{M}^{\mathcal{E}}\left(\sigma^{\prime}, f\right)=\sum_{G^{\prime} \in \mathcal{E}_{M^{\prime}}(G)} \iota_{M^{\prime}}\left(G, G^{\prime}\right) \widehat{S}_{\tilde{M}^{\prime}}^{\tilde{G}^{\prime}}\left(\sigma^{\prime}, f^{\prime}\right) .
$$

In case $G$ is quasisplit we define a "potentially stable" distribution

$$
S_{M}^{G}\left(M^{\prime}, \sigma^{\prime}, f\right)=I_{M}\left(\sigma^{\prime}, f\right)-\sum_{G^{\prime} \in \mathcal{E}_{M^{\prime}}^{0}(G)} \iota_{M^{\prime}}\left(G, G^{\prime}\right) \widehat{S}_{\tilde{M}^{\prime}}^{\tilde{G}^{\prime}}\left(\sigma^{\prime}, f^{\prime}\right) .
$$

In this case, we define the endoscopic distribution by the trivial relation

$$
I_{M}^{\mathcal{E}}\left(\sigma^{\prime}, f\right)=I_{M}\left(\sigma^{\prime}, f\right) .
$$

The left hand side of each equation is a section in $C^{\infty}\left(T_{G \text {-reg }}^{\prime}, M, \zeta\right)$, represented as the function of $\sigma^{\prime} \in \widetilde{T}_{G \text {-reg }}^{\prime}(\mathbb{R})$ attached to the given transfer factor $\Delta_{M}$ for $M^{\prime}$. Observe that the coefficient $\iota_{M^{\prime}}\left(G, G^{\prime}\right)$ vanishes unless $Z(\widehat{G})^{\Gamma}$ is of finite index in $Z\left(\widehat{G}^{\prime}\right)^{\Gamma}$, which is to say that the endoscopic datum $G^{\prime}$ is elliptic. The two sums may therefore be taken over the finite sets of elliptic endoscopic data in $\mathcal{E}_{M^{\prime}}(G)$ and $\mathcal{E}_{M^{\prime}}^{0}(G)$.

To complete the inductive definition, one still has to prove something serious in the special case that $G$ is quasisplit and $M^{\prime}=M^{*}$. In this case, we take $T^{*}=T^{\prime}=\widetilde{T}^{\prime}$ to be a maximal torus in $M^{*}$ over $\mathbb{R}$, and $\sigma^{*}=\sigma^{\prime}$ to be a strongly $G$-regular point in $T^{*}(\mathbb{R})$. The problem is to show that the distribution

$$
S_{M}^{G}(\sigma, f)=S_{M}^{G}\left(M^{*}, \sigma^{*}, f\right)
$$

on $G(\mathbb{R})$ is stable. (We follow the notation of [A11, $\S 2,3]$ here. In particular, $\sigma$ represents the stable conjugacy class in $G(\mathbb{R})$ that is the bijective preimage of the stable class in $G^{*}(\mathbb{R})$ represented by $\sigma^{*}$.) Only then would we have a linear form

$$
\widehat{S}_{M^{*}}^{G^{*}}\left(\sigma^{*}, f^{*}\right)=S_{M}^{G}(\sigma, f)
$$

on $\mathcal{S}\left(G^{*}, \zeta^{*}\right)$ that is the analogue for $\left(G^{*}, M^{*}\right)$ of the terms $\widehat{S}_{\tilde{M}^{\prime}}^{\tilde{G}^{\prime}}\left(\sigma^{\prime}, f^{\prime}\right)$ in $(1.8)$ and (1.9). Given the stability of (1.11), one has then to check that the stable distributions $S_{M^{*}}^{G^{*}}\left(\sigma^{*}, \cdot\right)$ on $G^{*}(\mathbb{R})$ satisfy the analogues of the conditions (1.6). This is straightforward. For example, any character $\omega^{*}$ on $G^{*}(\mathbb{R})$ transfers to a family of characters $\omega=\left\{\omega_{\iota}\right\}$ on the components $G_{\iota}(\mathbb{R})$. This transfers in turn to a character $\omega^{\prime}$ on any $\widetilde{G}^{\prime}(\mathbb{R})$, with the property that

$$
(\omega f)^{\prime}=\omega^{\prime} f^{\prime}, \quad f \in \mathcal{C}(G, \zeta) .
$$

The analogue for $G^{*}$ of the first relation in (1.6) then follows from the definitions (1.8) and (1.9) A16.

The discussion of this section has been quite brief, since the constructions are essentially those of [A11, §1-3]. We did not actually account for varying transfer factors in the definitions of [A11, §3] (and [A12, §4]). The reason was the mistaken view, expressed on p. 242 of A11, that the auxiliary data $\left(\widetilde{G}^{\prime}, \widetilde{\xi}^{\prime}\right)$ for the various $G^{\prime} \in \mathcal{E}_{M^{\prime}}(G)$ could all be chosen to have the same restriction to $M^{\prime}$. However, the discrepancy is minor. For a complete discussion of a more general situation, we refer the reader to the forthcoming paper $\mathrm{A} 15$. 
Theorem 1.1. Suppose that $M, M^{\prime}, T^{\prime}$ and $\sigma^{\prime} \in \widetilde{T}_{G-\mathrm{reg}}^{\prime}(\mathbb{R})$ are as above.

(a) If $G$ is arbitrary,

$$
I_{M}^{\mathcal{E}}\left(\sigma^{\prime}, f\right)=I_{M}\left(\sigma^{\prime}, f\right), \quad f \in \mathcal{C}(G, \zeta) .
$$

(b) If $G$ is quasisplit, the distribution

$$
f \longrightarrow S_{M}^{G}\left(M^{\prime}, \sigma^{\prime}, f\right), \quad f \in \mathcal{C}(G, \zeta),
$$

vanishes unless $M^{\prime}=M^{*}$, in which case it is stable.

The proof of this theorem will take up the rest of the paper. Notice that (a) is an assertion about the nonquasisplit case, since it is part of the definition (1.10) if $G$ is quasisplit. Observe also that (b) includes the stability assertion for quasisplit $G$ needed to complete the inductive definition above.

We shall say that $T^{\prime}$ is an $M$-image if any element in $T_{G \text {-reg }}^{\prime}(\mathbb{R})$ is an image [LS1, (1.3)] of some element in $M$. This is always the case if $G$ is quasisplit. If $T^{\prime}$ is not an $M$-image (so that $G$ is not quasisplit), the right hand side of (1.13) vanishes, by definition (1.4) and the basic properties of transfer factors. In this case, the local vanishing theorem [A11, Theorem 8.6] asserts that the left hand side of (1.13) is also zero. It is therefore enough for us to prove the theorem if $T^{\prime}$ is an $M$-image, an assumption we make henceforth.

\section{$\S 2$. Stabilization of the Differential EQUATions}

Theorem 1.1 will be proved by methods of analysis. The assertions (a) and (b) of the theorem may both be formulated as the vanishing of a function of $\sigma^{\prime}$. In each case, we will find that the relevant function satisfies a homogeneous linear boundary value problem. Our task will then be to show that the problem has no nonzero solution.

Boundary value problems are founded on differential equations. The invariant distributions (1.1) satisfy a family of differential equations, parametrized by elements $z$ in the center of the universal enveloping algebra. These equations are natural generalizations of the equations

$$
(z f)_{G}(\gamma)=\partial\left(h_{T}(z)\right) f_{G}(\gamma)
$$

that play a central role in Harish-Chandra's study of the invariant orbital integrals. We shall recall the generalization of (2.1) satisfied by the distributions $I_{M}(\gamma, f)$. We shall then review the results of A12, which provide a stabilization of these equations that is parallel to the constructions of the last section.

We have to remember that $G$ is a disjoint union of connected groups $G_{\iota}$. For any $\iota$, we write $\mathcal{Z}\left(G_{\iota}\right)$ for the center of the universal enveloping algebra of $\mathfrak{g}_{\iota}(\mathbb{C})$. (As usual, we denote the Lie algebra of a given algebraic group by a corresponding lower case Gothic letter.) We then form the quotient $\mathcal{Z}\left(G_{\iota}, \zeta_{\iota}\right)$ of $\zeta_{\iota}^{-1}$-covariants in $\mathcal{Z}\left(G_{\iota}\right)$. If the Schwartz space $\mathcal{C}\left(G_{\iota}, \zeta_{\iota}\right)$ is regarded as a space of sections on the line bundle on $G_{\iota}(\mathbb{R})$ defined by $\left(Z_{\iota}, \zeta_{\iota}\right), \mathcal{Z}\left(G_{\iota}, \zeta_{\iota}\right)$ becomes the algebra of biinvariant differential operators on this space. The inner twist $\psi_{\iota \kappa}$ from $G_{\kappa}$ to $G_{\iota}$ provides canonical isomorphisms from $\mathcal{Z}\left(G_{\kappa}\right)$ to $\mathcal{Z}\left(G_{\iota}\right)$ and $\mathcal{Z}\left(G_{\kappa}, \zeta_{\kappa}\right)$ to $\mathcal{Z}\left(G_{\iota}, \zeta_{\iota}\right)$. We can therefore attach canonical algebras $\mathcal{Z}(G)$ and $\mathcal{Z}(G, \zeta)$ of differential operators to $G$. They come with canonical isomorphisms from $\mathcal{Z}(G)$ to $\mathcal{Z}\left(G_{\iota}\right)$ and $\mathcal{Z}(G, \zeta)$ to $\mathcal{Z}\left(G_{\iota}, \zeta_{\iota}\right)$ for each $\iota$. 
For much of the rest of the paper, we shall treat $\gamma$ as a representative of a conjugacy class, rather than the conjugacy class itself. We can then form the centralizer

$$
T=M_{\gamma}=M_{\iota, \gamma}
$$

of $\gamma$ in the component $M_{\iota}$ of $M$ that contains $\gamma$. In fact, we shall generally fix a maximal torus $T$ over $\mathbb{R}$ in (some component of) $M$, and allow $\gamma$ to vary over points in $T_{G \text {-reg }}(\mathbb{R})$.

The differential equations (2.1) of course apply to the special case that $M=G$. The mapping

$$
z \longrightarrow h_{T}(z)=h_{T, G}(z)
$$

is the Harish-Chandra homomorphism attached to the torus $T=G_{\gamma}$. It can be regarded as an isomorphism from $\mathcal{Z}(G)$ onto the vector space of elements in the symmetric algebra on $\mathfrak{t}(\mathbb{C})$ that are invariant under the Weyl group

$$
W(G, T)=W\left(G_{\iota}, T\right), \quad T \subset G_{\iota},
$$

of $(G, T)$. We write $\partial\left(h_{T}(z)\right)$ as usual for the corresponding differential operator on $T(\mathbb{R})$ with constant coefficients, which is to say, with the property of being invariant (under translation by $T(\mathbb{R})$ ). The mapping $z \rightarrow \partial\left(h_{T}(z)\right.$ ) descends to an isomorphism from $\mathcal{Z}(G, \zeta)$ to the algebra of $W(G, T)$-invariant differential operators with constant coefficients on $C^{\infty}(T, \zeta)$, the space of sections of the line bundle $\mathcal{L}^{*}(T, \zeta)$ on $T(\mathbb{R})$ attached to $(Z, \zeta)$. This is how we will interpret Harish-Chandra's differential equations $(2.1)$.

The generalization of (2.1) was reviewed in [A12, §1]. It is a family of differential equations

$$
I_{M}(\gamma, z f)=\sum_{L \in \mathcal{L}(M)} \partial_{M}^{L}\left(\gamma, z_{L}\right) I_{L}(\gamma, f),
$$

in which $z$ again lies in $\mathcal{Z}(G, \zeta)$. For any $L, z \rightarrow z_{L}$ is the canonical injection of $\mathcal{Z}(G, \zeta)$ into $\mathcal{Z}(L, \zeta)$, and $\partial_{M}^{L}\left(\gamma, z_{L}\right)$ is a linear differential operator that depends only on $L$. It acts on $C^{\infty}\left(T_{G-\mathrm{reg}}, \zeta\right)$, the space of smooth sections of the restriction of the line bundle $\mathcal{L}^{*}(T, \zeta)$ to $T_{G \text {-reg }}(\mathbb{R})$. We include $\gamma$ in the notation because $\partial_{M}^{L}\left(\gamma, z_{L}\right)$ varies in general from point to point. However, in the special case that $L=M$, we note that

$$
\partial_{M}^{M}\left(\gamma, z_{M}\right)=\partial\left(h_{T, M}\left(z_{M}\right)\right)=\partial\left(h_{T}(z)\right) .
$$

The term with $L=M$ in (2.2) will eventually be decisive for us. Of the remaining terms, it is the one with $L=G$ that is important to understand, since we will be able to apply inductive arguments to the intermediate terms.

The notation for the differential operators $\partial_{M}^{G}(\gamma, z)$ was chosen deliberately to match that of the distributions $I_{M}(\gamma, f)=I_{M}^{G}(\gamma, f)$. In particular, one can try to stabilize $\partial_{M}^{G}(\gamma, z)$ in either $\gamma$ or $z$. It turns out that the two stabilizations are compatible. In other words, the analogue of Theorem 1.1 for $\partial_{M}^{G}(\gamma, z)$ has been shown to hold.

We fix an elliptic endoscopic datum $M^{\prime}$ for $M$, with maximal torus $T^{\prime} \subset M^{\prime}$ over $\mathbb{R}$, as in $\S 1$. We are assuming that $T^{\prime}$ is an $M$-image of $T$. Then there exists an $M$-admissible isomorphism from $T$ to $T^{\prime}$, by which we shall mean an $\mathbb{R}$-rational isomorphism of the form

$$
\phi=i^{-1} \circ \operatorname{Int}(h) \circ \psi_{M},
$$


where $i$ is an admissible embedding of $T^{\prime}$ into $M^{*}$ [LS1, (1.2)], $\psi_{M}$ is the inner twist from $M$ to $M^{*}$ compatible with the implicit identification of $\widehat{M}^{*}$ with $\widehat{M}$, and $h$ is an element in $M^{*}$ such that $h \psi_{M}(T) h^{-1}$ equals $i\left(T^{\prime}\right)$. We can use $\phi$ to transfer differential operators. This gives an isomorphism

$$
\partial \longrightarrow \bar{\partial}^{\prime}=\phi \partial=\left(\phi^{*}\right)^{-1} \circ \partial \circ \phi^{*}
$$

from the space of (linear) differential operators on $C^{\infty}\left(T_{G \text {-reg }}, \zeta\right)$ to the corresponding space of operators on $C^{\infty}\left(T_{G \text {-reg }}^{\prime}, \zeta\right)$. The isomorphism $\phi$ is uniquely determined up to the action of the real Weyl group $W_{\mathbb{R}}(M, T)$ of $M$ and $T$. (Recall that $W_{\mathbb{R}}(M, T)$ is the subgroup of elements in the full Weyl group $W(M, T)$ that are defined over $\mathbb{R}$. It in turn contains the subgroup $W(M(\mathbb{R}), T(\mathbb{R}))$ of elements induced from $M(\mathbb{R})$. These groups are of course taken relative the component $M_{\iota}$ of $M$ that contains $T$.) If $\partial$ is invariant under the action of $W_{\mathbb{R}}(M, T), \bar{\partial}^{\prime}$ does not depend on the choice of $\phi$.

Suppose that $\Delta_{M}$ is a transfer factor for $M$ and $M^{\prime}$. As we recall, $\Delta_{M}$ comes with an auxiliary datum $\left(\widetilde{M}^{\prime}, \widetilde{\xi}_{M}^{\prime}\right)$, an extension $\widetilde{T}^{\prime}$ of $T^{\prime}$, and a character $\widetilde{\zeta}^{\prime}$ on the preimage $\widetilde{Z}^{\prime}(F)$ of $Z(F)$ in $\widetilde{T}^{\prime}(F)$. One uses the internal structure of the $L$-embedding $\widetilde{\xi}_{M}^{\prime}$, together with symbols of differential operators, to construct an isomorphism $\bar{\partial}^{\prime} \rightarrow \partial^{\prime}$ between the spaces of (linear) differential operators on $C^{\infty}\left(T_{G \text {-reg }}^{\prime}(\mathbb{R}), \zeta\right)$ and $C^{\infty}\left(\widetilde{T}_{G \text {-reg }}^{\prime}(\mathbb{R}), \widetilde{\zeta}^{\prime}\right)$ respectively. Our essential concern is the composition $\partial \rightarrow \partial^{\prime}$ of the isomorphisms $\partial \rightarrow \bar{\partial}^{\prime}$ and $\bar{\partial}^{\prime} \rightarrow \partial^{\prime}$, and its restriction to the space of $W_{\mathbb{R}}(M, T)$-invariant differential operators on $C^{\infty}\left(T_{G \text {-reg }}, \zeta\right)$. It is this mapping that is compatible with endoscopic transfer. More precisely, suppose that $a$ is a $W_{\mathbb{R}}(M, T)$-invariant function in $C^{\infty}\left(T_{G \text {-reg }}, \zeta\right)$, and that $a^{\prime}=a_{\Delta_{M}}^{\prime}$ is the function in $C^{\infty}\left(\widetilde{T}_{G-\text { reg }}^{\prime}, \widetilde{\zeta}^{\prime}\right)$ defined by Langlands-Shelstad transfer. Then

$$
(\partial a)^{\prime}\left(\sigma^{\prime}\right)=\left(\partial^{\prime} a^{\prime}\right)\left(\sigma^{\prime}\right)
$$

for any $W_{\mathbb{R}}(M, T)$-invariant differential operator $\partial$ on $C^{\infty}\left(T_{G \text {-reg }}, \zeta\right)$ A12, Lemma $2.2]$.

The restriction of the mapping $\partial \rightarrow \partial^{\prime}$ to the $W_{\mathbb{R}}(M, T)$-invariant differential operators is again independent of $\phi$. It does depend implicitly on the transfer factor $\Delta_{M}$, through the associated datum $\left(\widetilde{M}, \widetilde{\xi}_{M}^{\prime}\right)$. However, its variance with $\Delta_{M}$ is compatible with the relations $(1.2)_{M}$ and $(1.3)_{M}$, a fact that is suggested by (2.4), and which is easy to check directly. We can therefore interpret $\partial \rightarrow \partial^{\prime}$ as a linear mapping from the space of $W_{\mathbb{R}}(M, T)$-invariant differential operators on $C^{\infty}\left(T_{G \text {-reg }}, \zeta\right)$ to the space of differential operators on the space of sections $C^{\infty}\left(T_{G \text {-reg }}^{\prime}, M, \zeta\right)$ of $\S 1$. However, we shall often treat $\partial^{\prime}$ as above, namely as the differential operator on $C^{\infty}\left(\widetilde{T}_{G-\text { reg }}^{\prime}, \widetilde{\zeta}^{\prime}\right)$ provided by an implicit choice of transfer factor $\Delta_{M}$, following the convention from $\S 1$.

There are two examples to bear in mind. The first is the case of a $W(G, T)$ invariant differential operator $\partial$ with constant coefficients on $C^{\infty}(T, \zeta)$. Then $\partial$ equals $\partial\left(h_{T}(z)\right)$, for a unique differential operator $z$ in $\mathcal{Z}(G, \zeta)$. Suppose that $G^{\prime}$ is an endoscopic datum for $G$ of which $M^{\prime}$ is a Levi subgroup. There is then a canonical injection $z \rightarrow \bar{z}^{\prime}$ from $\mathcal{Z}(G, \zeta)$ to $\mathcal{Z}\left(G^{\prime}, \zeta\right)$ such that

$$
\bar{\partial}\left(h_{T}(z)\right)^{\prime}=\partial\left(h_{T^{\prime}}\left(\bar{z}^{\prime}\right)\right) .
$$


There is also an injection $z \rightarrow z^{\prime}$ from $\mathcal{Z}(G, \zeta)$ to $\mathcal{Z}\left(\widetilde{G}^{\prime}, \widetilde{\zeta}^{\prime}\right)$, which depends on a choice of transfer factor $\Delta_{G}$ for $G$ and $G^{\prime}$, such that

$$
\partial\left(h_{T}(z)\right)^{\prime}=\partial\left(h_{\tilde{T}^{\prime}}\left(z^{\prime}\right)\right) .
$$

(See [A12, p. 84].) The mapping on the left hand side of (2.5) is taken relative to the restriction $\Delta_{M}$ of $\Delta_{G}$ to $M$.

The other example is the differential operator

$$
\partial_{M}^{G}(z)=\partial_{M}^{G}(\gamma, z), \quad z \in \mathcal{Z}(G, \zeta)
$$

with variable coefficients. It is easy to check that $\partial_{M}^{G}(z)$ is $W_{\mathbb{R}}(M, T)$-invariant [A12, Lemma 2.3]. It follows that for any transfer factor $\Delta_{M}$ for $M$ and $M^{\prime}$, $\partial_{M}^{G}(z)^{\prime}$ is a well defined differential operator on $C^{\infty}\left(\widetilde{T}_{G \text {-reg }}^{\prime}, \widetilde{\zeta}^{\prime}\right)$. We write

$$
\partial_{M}^{G}(z)^{\prime}=\partial_{M}^{G}\left(\sigma^{\prime}, z\right), \quad \sigma^{\prime} \in \widetilde{T}_{G-\mathrm{reg}}^{\prime}(\mathbb{R}) .
$$

This notation is motivated by (1.4). For if we apply the transfer identity (2.4) to $a(\gamma)=I_{M}(\gamma, f)$ and $\partial=\partial_{M}^{G}(z)$, we see that $\partial_{M}^{G}\left(\sigma^{\prime}, z\right)$ is an analogue for differential operators of the function $I_{M}\left(\sigma^{\prime}, f\right)$ in (1.4). In particular, $\partial_{M}^{G}\left(\sigma^{\prime}, z\right)$ can be regarded as the stabilization of $\partial_{M}^{G}(\gamma, z)$ in $\gamma$.

The stabilization of $\partial_{M}^{G}(\gamma, z)$ in $z$ follows the construction used to stabilize $I_{M}(\gamma, f)$ in $f$. In particular, it is formulated in terms of the set $\mathcal{E}_{M^{\prime}}(G)$. The inductive definitions (1.8)-(1.10) all have natural infinitesimal analogues, with the injection $z \rightarrow z^{\prime}$ playing the role of the transfer mapping $f \rightarrow f^{\prime}$. They give rise to differential operators on $C^{\infty}\left(\widetilde{T}_{G \text {-reg }}^{\prime}, \widetilde{\zeta}^{\prime}\right)$ that could be written naturally as $\partial_{M}^{G, \mathcal{E}}\left(\sigma^{\prime}, z\right)$ and $\delta_{M}^{G}\left(M^{\prime}, \sigma^{\prime}, z\right)$. However, we will not need to use this notation, since the infinitesimal analogue of Theorem 1.1 is already known. It is the main result of A12. We can therefore state the infinitesimal analogues of the definitions and the theorem together, following A12.

Proposition 2.1. For each $z \in \mathcal{Z}(G, \zeta)$, there is an identity

$$
\partial_{M}^{G}\left(\sigma^{\prime}, z\right)=\sum_{G^{\prime} \in \mathcal{E}_{M^{\prime}}(G)} \iota_{M^{\prime}}\left(G, G^{\prime}\right) \delta_{\tilde{M}^{\prime}}^{\tilde{G}^{\prime}}\left(\sigma^{\prime}, z^{\prime}\right),
$$

where

$$
\delta_{\tilde{M}^{\prime}}^{\tilde{G}^{\prime}}\left(\sigma^{\prime}, z^{\prime}\right), \quad G^{\prime} \in \mathcal{E}_{M^{\prime}}(G), \sigma^{\prime} \in \widetilde{T}_{G-\mathrm{reg}}^{\prime}(\mathbb{R}),
$$

is a differential operator on $C^{\infty}\left(\widetilde{T}_{G-\mathrm{reg}}^{\prime}(\mathbb{R}), \widetilde{\zeta}^{\prime}\right)$ that depends only on the quasisplit pair $\left(\widetilde{G}^{\prime}, \widetilde{M}^{\prime}\right)$ and the element $z^{\prime} \in \mathcal{Z}\left(\widetilde{G}^{\prime}, \widetilde{\zeta}^{\prime}\right)$.

See [A12, Theorem 3.1]. The summands in (2.7) are to be understood in the same manner as those in (1.8) and (1.9). Each represents a function of a variable transfer factor $\Delta_{M}$, with a specified value when $\Delta_{M}$ is the restriction to $M$ of the transfer factor $\Delta_{G}$ that defines the image $z^{\prime}$ of $z$. I would like to be able to say that this point was implicit in A12, but in truth, it was not considered at all. However, the proof from A12] does carry over without change.

In case $G$ is quasisplit, we sometimes write

$$
\delta_{M}^{G}(\sigma, z)=\delta_{M^{*}}^{G^{*}}\left(\sigma^{*}, z^{*}\right), \quad \sigma^{*} \in T_{G-\mathrm{reg}}^{*}(\mathbb{R}), z^{*} \in \mathcal{Z}\left(G^{*}, \zeta^{*}\right),
$$

for $\sigma$ as in (1.11), and $z$ the preimage of $z^{*}$ in $\mathcal{Z}(G, \zeta)$. This allows us to study stable distributions on the $K$-group $G$ rather than the connected group $G^{*}$. 
Proposition 2.1 represents the initial step towards a proof of Theorem 1.1. It implies, roughly speaking, that the assertions of the theorem are compatible with the differential equations (2.2). To formulate the implication more precisely, we have to take on a slightly stronger induction hypothesis than is implicit in the definitions of $\S 1$.

We first observe that the equation (2.2) can be combined with the original definition (1.4). Using the transfer identity (2.4), one finds [A12, (4.5)] that

$$
I_{M}\left(\sigma^{\prime}, z f\right)=\sum_{L \in \mathcal{L}(M)} \partial_{M}^{L}\left(\sigma^{\prime}, z_{L}\right) I_{L}\left(\sigma^{\prime}, f\right) .
$$

The functions $I_{L}\left(\sigma^{\prime}, f\right)$ of $\sigma^{\prime}$ here are to be treated as elements in the space of sections $C^{\infty}\left(T_{G \text {-reg }}^{\prime}, M, T\right)$ of the bundle $\mathcal{L}^{*}\left(T_{G \text {-reg }}^{\prime}, M, \zeta\right)$. This is consistent with the definition (1.4) (with $L$ in place of $M$, and any endoscopic datum $L^{\prime} \in \mathcal{E}_{M^{\prime}}(L)$ in place of $\left.M^{\prime}\right)$, since there is a natural bundle mapping from $\mathcal{L}\left(T_{G \text {-reg }}^{\prime}, L, \zeta\right)$ to $\mathcal{L}\left(T_{G \text {-reg }}^{\prime}, M, \zeta\right)$ given by the restriction $\Delta_{L} \rightarrow \Delta_{M}$ of transfer factors.

The theorem will ultimately be proved by a double induction, based on the two integers

$$
d_{\text {der }}=\operatorname{dim}\left(G_{\text {der }}\right)=\operatorname{dim}\left(G_{\iota, \text { der }}\right)
$$

and

$$
r_{\text {der }}=\operatorname{dim}\left(A_{M} \cap G_{\text {der }}\right)=\operatorname{dim}\left(A_{M_{\iota}} \cap G_{\iota, \text { der }}\right), \quad \iota \in \pi_{0}(G) .
$$

We will not adopt the full induction hypothesis until we have to in $\S 6$. However, we do assume henceforth that Theorem 1.1(b) holds if $\left(G, M, M^{\prime}\right)$ is replaced by any quasisplit triplet $\left(G_{1}, M_{1}, M_{1}^{\prime}\right)$ with

$$
\operatorname{dim}\left(G_{1, \mathrm{der}}\right) \leq d_{\text {der }}
$$

if $G$ is not quasisplit, and with

$$
\operatorname{dim}\left(G_{1, \mathrm{der}}\right)<d_{\text {der }}
$$

in case $G$ is quasisplit. This obviously includes our earlier ad hoc assumption that the summands in (1.7) and (1.8) be well defined. Proposition 2.1 then has the following corollary, which applies to operators $z \in \mathcal{Z}(G, \zeta)$ and functions $f \in \mathcal{C}(G, \zeta)$.

Corollary 2.2. (a) If $G$ is arbitrary,

$$
I_{M}^{\mathcal{E}}\left(\sigma^{\prime}, z f\right)=\sum_{L \in \mathcal{L}(M)} \partial_{M}^{L}\left(\sigma^{\prime}, z_{L}\right) I_{L}^{\mathcal{E}}\left(\sigma^{\prime}, f\right) .
$$

(b) If $G$ is quasisplit,

$$
S_{M}^{G}(\sigma, z f)=\sum_{L \in \mathcal{L}(M)} \delta_{M}^{L}\left(\sigma, z_{L}\right) S_{L}^{G}(\sigma, f),
$$

for $\sigma$ as in (1.11), while

$$
S_{M}^{G}\left(M^{\prime}, \sigma^{\prime}, z f\right)=\partial\left(h_{T}(z)\right)^{\prime} S_{M}^{G}\left(M^{\prime}, \sigma^{\prime}, f\right),
$$

if $M^{\prime} \neq M^{*}$.

The corollary is proved by combining the proposition with the original equations (1.8) and (1.9). See A12, Proposition 4.1].

The terms with $L \neq M$ in (2.8), (2.9) and (2.10) can be simplified by the formulas of descent satisfied by the various distributions. Let us recall these formulas. 
Suppose that the torus $T^{\prime} \subset M^{\prime}$ is not elliptic. Then it is contained in a proper Levi subgroup $M_{1}^{\prime} \subset M^{\prime}$. We are assuming that $T^{\prime}$ is an $M$-image. It follows easily that there is a proper Levi subgroup $M_{1}$ of $M$ for which $M_{1}^{\prime}$ represents an elliptic endoscopic datum. This allows us to identify $M^{\prime}$ with an element in $\mathcal{E}_{M_{1}^{\prime}}(M)$. Given a transfer factor $\Delta_{M}$ for $M$ and $M^{\prime}$, with auxiliary datum $\left(\widetilde{M}^{\prime}, \widetilde{\xi}_{M}^{\prime}\right)$, let $\Delta_{M_{1}}$ be the restricted transfer factor for $M_{1}$ and $M_{1}^{\prime}$, with auxiliary datum $\left(\widetilde{M}_{1}^{\prime}, \widetilde{\xi}_{M_{1}}^{\prime}\right)$. The distributions (1.4) and (1.8) then satisfy the formulas

$$
I_{M}\left(\sigma^{\prime}, f\right)=\sum_{G_{1} \in \mathcal{L}\left(M_{1}\right)} d_{M_{1}}^{G}\left(M, G_{1}\right) \widehat{I}_{M_{1}}^{G_{1}}\left(\sigma^{\prime}, f_{G_{1}}\right)
$$

and

$$
I_{M}^{\mathcal{E}}\left(\sigma^{\prime}, f\right)=\sum_{G_{1} \in \mathcal{L}\left(M_{1}\right)} d_{M_{1}}^{G}\left(M, G_{1}\right) \widehat{I}_{M_{1}}^{G_{1}, \mathcal{E}}\left(\sigma^{\prime}, f_{G_{1}}\right)
$$

of parabolic descent. The coefficient $d_{M_{1}}^{G}\left(M, G_{1}\right)$ here is the usual determinant, defined for example on p. 356 of [A4]. If $G$ is quasisplit and $M^{\prime}=M^{*}$, and the point $\sigma^{\prime}=\sigma^{*}$ is the image of a point $\sigma \in T_{G \text {-reg }}(\mathbb{R})$ in $M_{1}$, we have

$$
S_{M}^{G}(\sigma, f)=\sum_{G_{1} \in \mathcal{L}\left(M_{1}\right)} e_{M_{1}}^{G}\left(M, G_{1}\right) \widehat{S}_{M_{1}}^{G_{1}}\left(\sigma, f^{G_{1}}\right),
$$

where

$$
e_{M_{1}}^{G}\left(M, G_{1}\right)=d_{M}^{G}\left(M, G_{1}\right)\left|Z(\widehat{M})^{\Gamma} \cap Z\left(\widehat{G}_{1}\right)^{\Gamma} / Z(\widehat{G})^{\Gamma}\right|^{-1} .
$$

Finally, if $G$ is quasisplit but $M^{\prime} \neq M^{*}$, we have

$$
S_{M}^{G}\left(M^{\prime}, \sigma^{\prime}, f\right)=0 .
$$

The descent formulas above are all consequences of corresponding formulas A4, Proposition 7.1] for the distributions $I_{M}(\gamma, f)$. They were established in greater generality (and under more baroque induction hypotheses) in [A11, Theorem 7.1]. It is clear that with a slight extension of the induction hypothesis on $d_{\text {der }}$ above (namely, that it applies to both assertions (a) and (b) of Theorem 1.1), the formulas of descent imply Theorem 1.1 in the case where $T^{\prime} \subset M^{\prime}$ is not elliptic. It is also clear that they can be applied (with $L$ and $M$ in place of $M$ and $M_{1}$ ) to the summands $L \neq M$ in (2.8), (2.9) and (2.10).

\section{§3. Stabilization of Elliptic Boundary COnditions}

The next step is to study boundary conditions. We are interested in the behaviour of the distributions $I_{M}\left(\sigma^{\prime}, f\right), I_{M}^{\mathcal{E}}\left(\sigma^{\prime}, f\right)$, and $S_{M}^{G}\left(M^{\prime}, \sigma^{\prime}, f\right)$ of Theorem 1.1 as $\sigma^{\prime}$ approaches the boundary of $\widetilde{T}_{G \text {-reg }}^{\prime}(\mathbb{R})$ in $\widetilde{T}^{\prime}(\mathbb{R})$.

As functions of $\sigma^{\prime}$, the distributions do not extend smoothly across singular hypersurfaces in the complement of $\widetilde{T}_{G \text {-reg }}^{\prime}(\mathbb{R})$. However, the singularities are quite gentle. One can modify the functions in a simple way so that their derivatives in $\sigma^{\prime}$ remain bounded around any point in general position on a singular hypersurface. Moreover, there are explicit formulas for the jumps of their derivatives across the hypersurface.

Recall that $M, M^{\prime}$ and $T^{\prime}$ are fixed, while $\widetilde{T}^{\prime}$ is the extension of $T^{\prime}$ attached to the auxiliary datum $\left(\widetilde{M}^{\prime}, \widetilde{\zeta}^{\prime}\right)$ of a transfer factor $\Delta_{M}$. By a root of $T^{\prime}$, we shall mean the transfer

$$
\alpha^{\prime}=\widehat{\phi}^{-1}(\alpha)
$$


of some root $\alpha$ of $(G, T)$, where $T \subset M$ is a maximal torus over $\mathbb{R}$, and $\phi$ is an $M$-admissible isomorphism (2.3). (A root of $(G, T)$ is of course a root for the component $G_{\iota}$ of $G$ that contains $T$.) We can treat $\alpha^{\prime}$ as a character on either of the groups $T^{\prime}$ or $\widetilde{T}^{\prime}$, or as a linear form on either of the Lie algebras $\mathfrak{t}^{\prime}$ or $\widetilde{\mathfrak{t}}^{\prime}$. Then $\alpha^{\prime}$ is said to be real, imaginary or complex if its values on $\mathfrak{t}^{\prime}(\mathbb{R})$ have the corresponding property. We are interested in the case that the kernel of $\alpha^{\prime}$ in $\widetilde{T}^{\prime}(\mathbb{R})\left(\right.$ or $T^{\prime}(\mathbb{R})$ ) is a hypersurface, which is to say that it has codimension one. This rules out the complex roots. We shall treat the hypersurfaces attached to imaginary roots in this section, and real roots in the next.

We first recall an elementary point concerning the original distributions $I_{M}(\gamma, f)$. The weighted orbital integrals that are the primary components of these objects were normalized by the factor $\left|D_{G}(\gamma)\right|^{\frac{1}{2}}$ obtained from the absolute value of the Weyl discriminant A6, §1]. Harish-Chandra's jump conditions about imaginary roots require a more subtle normalization. We have therefore to introduce a familiar (but noncanonical) normalization for the functions of $\sigma^{\prime}$ in Theorem 1.1.

An imaginary root of $T^{\prime}$ comes from an imaginary root of $(G, T)$, which is in fact an imaginary root of $(M, T)$, since it vanishes on the split torus $A_{M}$. The renormalization concerns only the subset of imaginary roots of $T^{\prime}$ that are actually roots of $\left(M^{\prime}, T^{\prime}\right)$ in the usual sense. The set $R_{I}^{\prime}$ of imaginary roots of $\left(M^{\prime}, T^{\prime}\right)$ is a root system for $\left(M_{I}^{\prime}, T^{\prime}\right)$, where $M_{I}^{\prime}$ is the Levi subgroup of $M^{\prime}$ in which $T^{\prime}$ is $\mathbb{R}$-elliptic. The elements in $R_{I}^{\prime}$ divide the real vector space $i \mathfrak{t}^{\prime}(\mathbb{R})$ into chambers, on which the Weyl group $W_{I}^{\prime}$ of $R_{I}^{\prime}$ acts simply transitively. If $c$ is any chamber, we write $R_{c}^{\prime}$ for the corresponding set of positive roots in $R_{I}^{\prime}$. We then set

$$
\delta_{c}^{\prime}\left(\sigma^{\prime}\right)=\prod_{\alpha^{\prime} \in R_{c}^{\prime}}\left(1-\alpha^{\prime}\left(\sigma^{\prime}\right)^{-1}\right)\left|1-\alpha^{\prime}\left(\sigma^{\prime}\right)^{-1}\right|^{-1},
$$

a function that also equals

$$
\left|D_{M_{I}}\left(\sigma^{\prime}\right)\right|^{-\frac{1}{2}} \prod_{\alpha \in R_{c}^{\prime}}\left(1-\alpha^{\prime}\left(\sigma^{\prime}\right)^{-1}\right),
$$

and is defined for any strongly $M_{I}^{\prime}$-regular point $\sigma^{\prime}$ in $\widetilde{T}^{\prime}(\mathbb{R})$. The mapping

$$
a^{\prime} \longrightarrow a_{c}^{\prime}, \quad a^{\prime} \in C^{\infty}\left(T^{\prime}, M, \zeta\right),
$$

in which

$$
a_{c}^{\prime}\left(\sigma^{\prime}\right)=\delta_{c}^{\prime}\left(\sigma^{\prime}\right) a^{\prime}\left(\sigma^{\prime}\right), \quad \sigma^{\prime} \in \widetilde{T}_{G-\mathrm{reg}}^{\prime}(\mathbb{R}),
$$

is easily seen to be a linear automorphism of the space $C^{\infty}\left(T^{\prime}, M, \zeta\right)$ of sections of the line bundle $\mathcal{L}^{*}\left(T^{\prime}, M, \zeta\right)$. The jump conditions of interest apply to the images under this mapping of the functions of Theorem 1.1.

We fix an arbitrary imaginary root $\alpha^{\prime}$ of $T^{\prime}$. We also fix a point in general position in the corresponding singular hypersurface

$$
\left(\widetilde{T}^{\prime}\right)^{\alpha^{\prime}}(\mathbb{R})=\left\{\sigma_{1}^{\prime} \in \widetilde{T}^{\prime}(\mathbb{R}): \alpha^{\prime}\left(\sigma_{1}^{\prime}\right)=1\right\},
$$

which we may as well continue to denote by $\sigma^{\prime}$. Given $\sigma^{\prime}$, we then choose a small open neighbourhood $\widetilde{U}^{\prime}$ of the stable class of $\sigma^{\prime}$ in the set of strongly $G$-regular stable conjugacy classes $\Delta_{G \text {-reg }}\left(\tilde{M}^{\prime}\right)$ in $\tilde{M}^{\prime}(\mathbb{R})$. The distributions of $\S 1$ can of course be defined for a class $\delta^{\prime} \in \widetilde{U}^{\prime}$ (in place of a point in $\widetilde{T}_{G-\text { reg }}(\mathbb{R})$ ). 
Lemma 3.1. Suppose that

$$
E_{M}\left(\delta^{\prime}, f\right), \quad f \in \mathcal{C}(G, \zeta)
$$

is one of the families of distributions $I_{M}\left(\delta^{\prime}, f\right), I_{M}^{\mathcal{E}}\left(\delta^{\prime}, f\right)$ or $S_{M}^{G}\left(M^{\prime}, \delta^{\prime}, f\right)$, defined as in $\S 1$ for the transfer factor $\Delta_{M}$ and the classes $\delta^{\prime} \in \widetilde{U}^{\prime}$. Then there is a function $e_{f}^{\prime}$ in $\mathcal{S}\left(\widetilde{M}^{\prime}, \widetilde{\zeta}^{\prime}\right)$ such that

$$
E_{M}\left(\delta^{\prime}, f\right)=e_{f}^{\prime}\left(\delta^{\prime}\right), \quad \delta^{\prime} \in \widetilde{U}^{\prime} .
$$

Proof. Let $U^{\prime} \subset \Delta_{G \text {-reg }}\left(M^{\prime}\right)$ be the projection of $\widetilde{U}^{\prime}$ onto $M^{\prime}(\mathbb{R})$, and let $U$ be the set of conjugacy classes $\gamma \in \Gamma_{G \text {-reg }}(M)$ in $M(\mathbb{R})$ of which some element in $U^{\prime}$ is an image. Then $U$ is a finite disjoint union of sets $U\left(\gamma_{1}\right)$, where $\gamma_{1}$ ranges over a set of elements in $M(\mathbb{R})$ of which the projection of $\sigma^{\prime}$ onto $M^{\prime}(\mathbb{R})$ is an image, and $U\left(\gamma_{1}\right)$ is a small open neighbourhood of the class of $\gamma_{1}$ in $\Gamma_{G \text {-reg }}(M)$. Any class in $U\left(\gamma_{1}\right)$ has a representative $\gamma \in M(\mathbb{R})$ that commutes with $\gamma_{1}$, and is close to $\gamma_{1}$. It follows easily from the general position of $\sigma^{\prime}$ in $\left(\widetilde{T}^{\prime}\right)^{\alpha^{\prime}}(\mathbb{R})$ that the centralizer of each $\gamma_{1}$ in (its component in) $G$ is contained in $M$. If we apply [A4, (2.3)] to each of the points $\gamma_{1}$, we deduce that there is a function $e_{f}$ in $\mathcal{I}(M, \zeta)$ such that

$$
I_{M}(\gamma, f)=e_{f}(\gamma), \quad \gamma \in U .
$$

It follows from the definition (1.4) that

$$
I_{M}\left(\delta^{\prime}, f\right)=e_{f}^{\prime}\left(\delta^{\prime}\right), \quad \delta^{\prime} \in \widetilde{U}^{\prime},
$$

where $e_{f}^{\prime}$ is the transfer of $e_{f}$ to a function in $\mathcal{S}\left(\widetilde{M}^{\prime}, \widetilde{\zeta}^{\prime}\right)$. This establishes (3.3) in case $E_{M}\left(\delta^{\prime}, f\right)$ equals $I_{M}\left(\delta^{\prime}, f\right)$.

Suppose then that $E_{M}\left(\delta^{\prime}, f\right)$ equals either $I_{M}^{\mathcal{E}}\left(\delta^{\prime}, f\right)$ or $S_{M}^{G}\left(M^{\prime}, \delta^{\prime}, f\right)$. In each of these cases, we deduce that (3.3) holds for some function $e_{f}^{\prime}$ in $\mathcal{S}\left(\widetilde{M}^{\prime}, \widetilde{\zeta}^{\prime}\right)$ by combining the case we have just established with an application of the appropriate induction hypothesis to the summands in either (1.8) or (1.9).

Remark. It follows from the definitions that $e_{f}^{\prime}$ is compatible with the underlying transfer factor $\Delta_{M}$, in the sense that it represents an element in $C^{\infty}\left(T^{\prime}, M, \zeta\right)$. In fact, one can show that it is the image of a function $e_{f} \in \mathcal{I}(M, \zeta)$, just as in the special case that $E_{M}\left(\delta^{\prime}, f\right)$ equals $I_{M}\left(\delta^{\prime}, f\right)$.

The relation (3.3) we have just established reduces the singularities around $\sigma^{\prime}$ of the distributions of $\S 1$ to corresponding singularities of stable orbital integrals. The explicit description of the latter was one of the initial steps taken by Shelstad S1 in developing a theory of endoscopy for real groups.

Since the fixed root $\alpha^{\prime}$ is imaginary, its corresponding coroot is of the form

$$
\left(\alpha^{\prime}\right)^{\vee}=i H_{\alpha}^{\prime}
$$

for a vector $H_{\alpha}^{\prime}=H_{\alpha^{\prime}}$ in the Lie algebra $\widetilde{\mathfrak{t}}^{\prime}(\mathbb{R})$. We write

$$
j_{\alpha^{\prime}}\left(a^{\prime}\left(\sigma^{\prime}\right)\right)=\lim _{\theta \rightarrow 0+} a^{\prime}\left(\sigma^{\prime} \exp \theta H_{\alpha}^{\prime}\right)-\lim _{\theta \rightarrow 0-} a^{\prime}\left(\sigma^{\prime} \exp \theta H_{\alpha}^{\prime}\right),
$$

for any section $a^{\prime} \in C^{\infty}\left(T_{G \text {-reg }}^{\prime}, M, \zeta\right)$ for which the two half limits exist. Our concern is the jump attached to the section

$$
a^{\prime}(\cdot)=D_{c}^{\prime} e_{c}^{\prime}(\cdot)
$$

where $e$ is any function in $\mathcal{I}(M, \zeta)$ and $D_{c}^{\prime}$ is an invariant differential operator on $C^{\infty}\left(T^{\prime}, M, \zeta\right)$. The existence of the two sided limits in this case is an immediate 
consequence of a general theorem of Harish-Chandra on invariant orbital integrals. The difference represents the obstruction to being able to extend $D_{c}^{\prime} e_{c}^{\prime}$ to a continuous function at $\sigma^{\prime}$. The results of Shelstad, which follow similar results of Harish-Chandra, give precise formulas for this difference. We shall apply them to the functions

$$
e^{\prime}\left(\delta^{\prime}\right)=e_{f}^{\prime}\left(\delta^{\prime}\right)=E_{M}\left(\delta^{\prime}, f\right)
$$

of the last lemma.

The results of Harish-Chandra and Shelstad are expressed in terms of Cayley transforms. Suppose that $\alpha^{\prime}$ is the transfer of the imaginary root $\alpha$ of $(M, T)$ under an $M$-admissible isomorphism $\phi$ from $T$ to $T^{\prime}$. The projection of $\sigma^{\prime}$ onto $T^{\prime}$ then equals $\phi(\gamma)$, where $\gamma$ is a point in general position in the singular hypersurface

$$
T^{\alpha}(\mathbb{R})=\{\gamma \in T(\mathbb{R}): \alpha(\gamma)=1\} .
$$

The centralizer $G_{\gamma}$ of $\gamma$ (in its component $G_{\iota}$ ) is a connected reductive group over $\mathbb{R}$, whose derived group $G_{\gamma, \text { der }}$ is three dimensional. Recall that $\alpha$ is said to be compact or noncompact according to whether the group $G_{\gamma, \operatorname{der}}(\mathbb{R})$ has the corresponding property.

Suppose that $\alpha$ is noncompact. We will again write

$$
\alpha^{\vee}=i H_{\alpha}
$$

for a vector $H_{\alpha}$ in the Lie algebra of $T(\mathbb{R}) \cap G_{\gamma, \text { der }}(\mathbb{R})$. The group $G_{\gamma, \text { der }}$ is now isomorphic over $\mathbb{R}$ to either $S L(2)$ or $P G L(2)$. Let $T_{\alpha}$ be a maximal torus in $G_{\gamma}$ over $\mathbb{R}$ such that the Lie algebras of $T_{\alpha} \cap G_{\gamma, \text { der }}$ and $T \cap G_{\gamma \text {,der }}$ are orthogonal with respect to the Killing form on $\mathfrak{g}_{\gamma, \text { der }}$. Then $T_{\alpha} \cap G_{\gamma, \text { der }}$ is a (one-dimensional) split torus in $G_{\gamma, \text { der }}$. We write $M_{\alpha}$ for a maximal Levi $(K-)$ subgroup of $M$ whose intersection with $G_{\gamma, \text { der }}$ coincides with that of $T_{\alpha}$. By a Cayley transform, we mean an isomorphism from $T$ to $T_{\alpha}$ of the form

$$
C_{\alpha}=\operatorname{Int}\left(s_{\alpha}\right), \quad s_{\alpha} \in G_{\gamma, \mathrm{der}} .
$$

If $C_{\alpha}$ is fixed, we write $\beta$ for the transfer of $\alpha$ by $C_{\alpha}$ to a root of $\left(G_{\gamma}, T_{\alpha}\right)$. We then form the vector

$$
Z_{\alpha}=H_{\beta}=\beta^{\vee}=\left(d C_{\alpha}\right)\left(\alpha^{\vee}\right)
$$

in the Lie algebra of $T_{\alpha}(\mathbb{R}) \cap G_{\gamma, \text { der }}(\mathbb{R})$.

The pair $\left(T_{\alpha}, C_{\alpha}\right)$ is of course not uniquely determined by $\alpha$ and $\gamma$. For example, we can always replace $C_{\alpha}$ by its complex conjugate

$$
\bar{C}_{\alpha}=C_{\alpha} w_{\alpha},
$$

where $w_{\alpha}$ is the reflection in $T$ about $\alpha$. We set $d_{\alpha}$ equal to 1 or 2 , according to whether or not $w_{\alpha}$ lies in the subgroup $W(M(\mathbb{R}), T(\mathbb{R}))$ of $W_{\mathbb{R}}(M, T)$. Then $\bar{C}_{\alpha}$ is $G_{\gamma}(\mathbb{R})$-conjugate to $C_{\alpha}$ if and only if $d_{\alpha}=1$. In fact, $d_{\alpha}$ equals the number of $G_{\gamma}(\mathbb{R})$ conjugacy classes of pairs $\left(T_{\alpha}, C_{\alpha}\right)$ attached to $\alpha$ and $\gamma$. It also equals the number of $G_{\gamma}(\mathbb{R})$-orbits in $\mathfrak{g}_{\gamma}(\mathbb{R})$ represented by the pair $\left\{\alpha^{\vee},-\alpha^{\vee}\right\}$, or equivalently, the number of $G_{\gamma}(\mathbb{R})$-conjugacy classes represented by the pair $\left\{\gamma \exp \left( \pm \theta H_{\alpha}\right)\right\}$ defined for any $\theta \neq 0$. These conditions are well known. They are readily verified with an inspection of the group $P G L(2, \mathbb{R})$ and its abelian extensions.

The notions above have obvious analogues if the $K$-group $M$ is replaced by the connected group $M^{\prime}$, and $\alpha$ is replaced by our fixed imaginary root $\alpha^{\prime}$. If $\alpha^{\prime}$ 
belongs to the set $R_{I, \mathrm{nc}}^{\prime}$ of noncompact roots in $R_{I}^{\prime}$, we can form objects $T_{\alpha}^{\prime}=T_{\alpha^{\prime}}^{\prime}$, $M_{\alpha}^{\prime}=M_{\alpha^{\prime}}^{\prime}, w_{\alpha}^{\prime}=w_{\alpha^{\prime}}, C_{\alpha}^{\prime}=C_{\alpha^{\prime}}, \beta^{\prime}$ and $Z_{\alpha}^{\prime}=Z_{\alpha^{\prime}}$, as above. In this case, we write

$$
\sigma_{\alpha}^{\prime}(r)=\sigma^{\prime} \exp \left(r Z_{\alpha}^{\prime}\right)
$$

for any small real number $r \neq 0$. We also write $c_{\alpha}=c_{\alpha}^{\prime}$ for the chamber in $i \mathfrak{t}_{\alpha}^{\prime}(\mathbb{R})$ defined by the system $R_{c_{\alpha}}^{\prime}$ of positive imaginary roots that are mapped by the transpose of $\operatorname{Ad}\left(C_{\alpha}^{\prime}\right)$ to $R_{c}^{\prime}$.

We will now state the jump conditions. They include a vanishing assertion, which pertains to the set $R_{I, \mathrm{nc}}^{\prime}\left(\alpha^{\prime}\right)$ of noncompact roots in $R_{I}^{\prime}$ of the form $w^{\prime} \alpha^{\prime}$, for some $w^{\prime} \in W_{I}^{\prime}$, and the set $R_{I, \mathrm{nc}}\left(\alpha^{\prime}\right)$ of noncompact roots of $(M, T)$ (for some $T$ ) that transfer to $\alpha^{\prime}$.

Proposition 3.2. Suppose that $E_{M}\left(\delta^{\prime}, f\right)$ represents one of the three families of distributions $I_{M}\left(\delta^{\prime}, f\right), I_{M}^{\mathcal{E}}\left(\delta^{\prime}, f\right)$ or $S_{M}^{G}\left(M^{\prime}, \delta^{\prime}, f\right)$, as in Lemma 3.1.

(i) Suppose that $\alpha^{\prime}$ is a noncompact root in $R_{I}^{\prime}$ that is the transfer of a noncompact root $\alpha$ of $(M, T)$, as above. Then

$$
j_{\alpha^{\prime}}\left(D_{c}^{\prime} E_{M, c}\left(\sigma^{\prime}, f\right)\right)=\lim _{r \rightarrow 0} D_{c, \alpha}^{\prime} E_{M, c_{\alpha}}\left(\sigma_{\alpha}^{\prime}(r), f\right),
$$

where $D_{c, \alpha}^{\prime}=D_{c, \alpha^{\prime}}^{\prime}$ is an invariant differential operator on $C^{\infty}\left(T_{\alpha}^{\prime}, M, \zeta\right)$ attached to the invariant differential operator $D_{c}^{\prime}$ on $C^{\infty}\left(T^{\prime}, M, \zeta\right)$ and the chamber $c$.

(ii) Suppose that one of the two sets $R_{I, \mathrm{nc}}^{\prime}\left(\alpha^{\prime}\right)$ or $R_{I, \mathrm{nc}}\left(\alpha^{\prime}\right)$ is empty. Then

$$
j_{\alpha^{\prime}}\left(D_{c}^{\prime} E_{M, c}\left(\sigma^{\prime}, f\right)\right)=0 .
$$

Proof. Applying Lemma 3.1, we write

$$
j_{\alpha^{\prime}}\left(D_{c}^{\prime} E_{M, c}\left(\sigma^{\prime}, f\right)\right)=j_{\alpha^{\prime}}\left(D_{c}^{\prime} e_{f, c}^{\prime}\left(\sigma^{\prime}\right)\right),
$$

for a function $e_{f}^{\prime} \in \mathcal{S}\left(\widetilde{M}^{\prime}, \widetilde{\zeta}^{\prime}\right)$. The required assertions then reduce to statements about singularities of stable orbital integrals on $\tilde{M}^{\prime}(\mathbb{R})$.

The reduction (3.5) holds without restriction on $\alpha^{\prime}$. If $\alpha^{\prime}$ belongs to $R_{I}^{\prime}$, it gives rise to an associated Cayley transform on $M^{\prime}$. In this case, we can use Shelstad's result [S1, Lemma 4.3] for stable orbital integrals on $\widetilde{M}^{\prime}(\mathbb{R})$. It implies that

$$
j_{\alpha^{\prime}}\left(D_{c}^{\prime} e_{f, c}^{\prime}\left(\sigma^{\prime}\right)\right)=\lim _{r \rightarrow 0} D_{c, \alpha^{\prime}}^{\prime} e_{f, c_{\alpha}}^{\prime}\left(\sigma_{\alpha}^{\prime}(r)\right),
$$

for an invariant differential operator $D_{c, \alpha^{\prime}}^{\prime}$ on $C^{\infty}\left(\widetilde{T}_{G \text {-reg }}^{\prime}, \widetilde{\zeta}^{\prime}\right)$ attached to $D_{c}^{\prime}$ and $\Delta_{M}$. If $\alpha^{\prime}$ is also the transfer of a noncompact root $\alpha$ of $(M, T)$, one sees easily that the operator $D_{\alpha, c}^{\prime}=D_{\alpha^{\prime}, c}^{\prime}$ represents an element in the space $C^{\infty}\left(T_{\alpha}^{\prime}, M_{\alpha}, \zeta\right)$. Part (i) follows.

Part (ii) really contains two assertions. For the first, we recall that any conjugacy class in the stable class of $\sigma^{\prime}$ in $\widetilde{M}^{\prime}(\mathbb{R})$ can be represented by a point $w^{\prime} \sigma^{\prime}$ in $\left(\widetilde{T}^{\prime}\right)^{w^{\prime} \alpha^{\prime}}(\mathbb{R})$ for some $w^{\prime} \in W_{I}^{\prime}$. The orbital integral of any function in $\mathcal{C}\left(\widetilde{M}^{\prime}, \widetilde{\zeta}^{\prime}\right)$ extends to a smooth function around this point, unless $w^{\prime} \alpha^{\prime}$ is a noncompact root in $R_{I}^{\prime}$. The jump (3.4) therefore vanishes if the set $R_{I, \mathrm{nc}}^{\prime}\left(\alpha^{\prime}\right)$ is empty, and in particular, if $\alpha^{\prime}$ is not a root of $\left(M^{\prime}, T^{\prime}\right)$. For the second assertion of (ii), we assume that $R_{I, \mathrm{nc}}\left(\alpha^{\prime}\right)$ is empty. We can also assume that $R_{I, \mathrm{nc}}^{\prime}\left(\alpha^{\prime}\right)$ is nonempty, since we have just seen that the jump would otherwise vanish. Since the quotient

$$
\left(\delta_{c}^{\prime} \circ w^{\prime}\right)\left(\delta_{c}^{\prime}\right)^{-1}=\left(\delta_{\left(w^{\prime}\right)^{-1} c}^{\prime}\right)\left(\delta_{c}^{\prime}\right)^{-1}
$$


extends to a smooth function on $\widetilde{T}^{\prime}(\mathbb{R})$, for any element $w^{\prime} \in W_{I}^{\prime}, e_{f, c}^{\prime}$ extends to a smooth function around $w^{\prime} \sigma^{\prime}$ if it extends to a smooth function around $\sigma^{\prime}$. We may therefore assume that the root $\alpha^{\prime}$ itself is noncompact, and hence that (3.6) holds. But our assumption that $\alpha^{\prime}$ transfers only to compact roots of $M$ implies that the point $\sigma_{\alpha}^{\prime}(r)$ in $\widetilde{T}_{\alpha}^{\prime}(\mathbb{R})$ on the right hand side of (3.6) is not an image of any point in $M(\mathbb{R})$. This means that $G$ is not quasisplit, and hence that $E_{M}\left(\delta^{\prime}, f\right)$ equals either $I_{M}\left(\delta^{\prime}, f\right)$ or $I_{M}^{\mathcal{E}}\left(\delta^{\prime}, f\right)$. It then follows, from either the definition (1.4) or the local vanishing property [A11, Theorem 8.6], that the right hand side of (3.6) itself vanishes. So therefore does the jump on the left hand side. The required assertion follows from (3.5).

We supplement the proposition with a few elementary remarks. Suppose for a moment that $\alpha^{\prime}$ is a variable index, as in (3.1). If the derived group of $\widetilde{M}^{\prime}$ is simply connected, the linear form

$$
\rho_{c}=\frac{1}{2} \sum_{\alpha^{\prime} \in R_{c}^{\prime}} \alpha^{\prime}
$$

lifts to a character

$$
\xi_{c}^{\prime}\left(\exp H^{\prime}\right)=e^{\rho_{c}\left(H^{\prime}\right)}, \quad H^{\prime} \in \widetilde{\mathfrak{t}}^{\prime}(\mathbb{C}),
$$

on $\widetilde{T}^{\prime}(\mathbb{C})$. In this case, the product

$$
\left(\xi_{c}^{\prime} \delta_{c}^{\prime}\right)(\exp H)=\prod_{\alpha^{\prime} \in R_{c}^{\prime}}\left(e^{\frac{1}{2} \alpha^{\prime}\left(H^{\prime}\right)}-e^{-\frac{1}{2} \alpha^{\prime}\left(H^{\prime}\right)}\right)\left|1-e^{-\alpha^{\prime}\left(H^{\prime}\right)}\right|^{-1}
$$

is a locally constant function on $\widetilde{T}_{\text {reg }}^{\prime}(\mathbb{R})$, since $\alpha^{\prime}\left(H^{\prime}\right)$ is purely imaginary for $H^{\prime} \in \widetilde{\mathfrak{t}}^{\prime}(\mathbb{R})$. Since $\xi_{c}^{\prime}$ is a smooth function on $\widetilde{T}^{\prime}(\mathbb{R})$, the singularities of the function $E_{M, c}(\cdot, f)$ of the proposition are similar to those of the product of the original function $E_{M}(\cdot, f)$ with the locally constant function $\xi_{c}^{\prime} \delta_{c}^{\prime}$. It is in terms of this second normalization (or rather, a local version that applies to the case that $\tilde{M}_{\text {der }}^{\prime}$ is not simply connected) that Harish-Chandra first expressed the jump conditions satisfied by invariant orbital integrals [H2, Theorem 9.1]. Since its normalizing factor is locally constant, this normalization offers the minor simplification of commuting with differential operators on $\widetilde{T}^{\prime}(\mathbb{R})$. The first normalization satisfies the slightly more complicated formula

$$
\left(D^{\prime} a^{\prime}\right)_{c}=D_{c}^{\prime} a_{c}^{\prime}, \quad a^{\prime} \in C^{\infty}\left(T^{\prime}, M, \zeta\right),
$$

where $D^{\prime} \rightarrow D_{c}^{\prime}$ is the isomorphism of the space of invariant differential operators on $C^{\infty}\left(T^{\prime}, M, \zeta\right)$ induced by the mapping

$$
H^{\prime} \longrightarrow H^{\prime}+\rho_{c}\left(H^{\prime}\right) I, \quad H^{\prime} \in \widetilde{\mathfrak{t}}^{\prime}(\mathbb{C}) .
$$

(See [S1, p. 24].)

Suppose again that $\alpha^{\prime}$ and $\sigma^{\prime}$ are fixed as in the proposition. Replacing $\alpha^{\prime}$ by $\left(-\alpha^{\prime}\right)$, if necessary, we can assume that $\alpha^{\prime}$ belongs to $R_{c}^{\prime}$. Consider the original normalizing factor $\delta_{c}^{\prime}\left(\sigma^{\prime} \exp \theta H_{\alpha}^{\prime}\right)$ as a function of $\theta$ around 0 . The term corresponding to $\alpha^{\prime}$ in the product (3.1) that defines $\delta_{c}^{\prime}$ is then

$$
\left(1-\alpha^{\prime}\left(\sigma^{\prime} \exp \theta H_{\alpha}^{\prime}\right)^{-1}\right)\left|1-\alpha^{\prime}\left(\sigma^{\prime} \exp \theta H_{\alpha}^{\prime}\right)^{-1}\right|^{-1} \text {. }
$$

Since

$$
\alpha^{\prime}\left(H_{\alpha}^{\prime}\right)=\alpha^{\prime}\left(i^{-1}\left(\alpha^{\prime}\right)^{\vee}\right)=-2 i
$$


this equals

$$
\left(1-e^{2 i \theta}\right)\left|1-e^{2 i \theta}\right|^{-1}=e^{i \theta} \varepsilon(\theta),
$$

where

$$
\varepsilon(\theta)=-i \cdot \operatorname{sign} \theta .
$$

The other factors in (3.1) all extend to smooth functions of $\theta$ at $\theta=0$. We can therefore normalize $E_{M}\left(\sigma^{\prime} \exp \theta H_{\alpha}^{\prime}, f\right)$ by multiplying it by the locally constant function $\varepsilon(\theta)$. We conclude that there is a linear mapping $D^{\prime} \rightarrow D_{\alpha}^{\prime}$ between the spaces of invariant differential operators on $C^{\infty}\left(T^{\prime}, M, \zeta\right)$ and $C^{\infty}\left(T_{\alpha}^{\prime}, M, \zeta\right)$ such that the assertions of the proposition hold with $\varepsilon(\theta) E_{M}\left(\sigma^{\prime} \exp \theta H_{\alpha}^{\prime}, f\right), E_{M}\left(\sigma_{\alpha}^{\prime}(r), f\right), D^{\prime}$ and $D_{\alpha}^{\prime}$ in place of $E_{M, c}\left(\sigma^{\prime} \exp \theta H_{\alpha}^{\prime}, f\right), E_{M, c_{\alpha}}\left(\sigma_{\alpha}^{\prime}(r), f\right), D_{c}^{\prime}$ and $D_{c, \alpha}^{\prime}$, respectively. This is essentially the normalization of Harish-Chandra mentioned above. It is simpler, but has the disadvantage of applying only locally in a neighbourhood of $\sigma^{\prime}$.

We will not need to know much about the differential operator $D_{c, \alpha}^{\prime}$ in formula (3.4), but it is easy to describe in terms of $D_{c}^{\prime}$. It satisfies the formula

$$
D_{c, \alpha}^{\prime}=k_{\alpha}^{\prime}\left(C_{\alpha}^{\prime} D^{\prime}\right)_{c_{\alpha}}
$$

where $k_{\alpha}^{\prime}=k_{\alpha^{\prime}}$ is a constant, $D^{\prime}$ is the preimage of $D_{c}^{\prime}$ under the mapping $D^{\prime} \rightarrow D_{c}^{\prime}$, and $C_{\alpha}^{\prime} D^{\prime}$ is the differential operator on $C^{\infty}\left(T_{\alpha}^{\prime}, M, \zeta\right)$ obtained from $D^{\prime}$ and the isomorphism $C_{\alpha}^{\prime}$. (See [S1, p. 24].) The operators $D^{\prime}$ and $D_{\alpha}^{\prime}$ that apply to the normalization defined by $\varepsilon(\theta)$ satisfy the simpler relation

$$
D_{\alpha}^{\prime}=k_{\alpha}^{\prime}\left(C_{\alpha}^{\prime} D^{\prime}\right) .
$$

The function $E_{M}\left(\sigma_{\alpha}^{\prime}(r), f\right)$ that gives the jump in this second normalization is symmetric in $r$ about 0 . It follows that if $D^{\prime}$ is antisymmetric with respect to reflection about $\alpha^{\prime}$ in $\widetilde{T}^{\prime}(\mathbb{R})$, then

$$
\lim _{r \rightarrow 0} D_{\alpha}^{\prime} E_{M}\left(\sigma_{\alpha}^{\prime}(r), f\right)=0 .
$$

In this case, the function

$$
D^{\prime}\left(\varepsilon(\theta) E_{M}\left(\sigma^{\prime} \exp \theta H_{\alpha}^{\prime}, f\right)\right), \quad \theta \neq 0,
$$

extends continuously about $\theta=0$. Such matters are of course well known. We mention them only to clarify some aspects of the discussion of the next section.

The constant $k_{\alpha}^{\prime}$ depends on the choices of measures in the weighted orbital integrals that are the source of the various distributions. We have implicitly normalized the measures by the conventions of Harish-Chandra [H2, \$7] rather than those of Shelstad [S1, §4]. (See [A6, §1].) For example, if $T$ is elliptic in $M$, the Haar measure on $T(\mathbb{R})$ is given by the measure on the group $A_{M}(\mathbb{R})^{0} \cong \mathfrak{a}_{M}$ attached to a fixed Euclidean norm $\|\cdot\|$ on the vector space $\mathfrak{a}_{M}$, and the normalized Haar measure on the compact group $T(\mathbb{R}) / A_{M}(\mathbb{R})^{0}$. The norms $\|\cdot\|$ on the various vector spaces $\mathfrak{a}_{M}, \mathfrak{a}_{M_{\alpha}}, \mathfrak{a}_{\tilde{M}^{\prime}}$, etc. are understood to have been chosen so that they satisfy all the natural compatibility conditions.

It is easy to describe the value of $k_{\alpha}^{\prime}$, with these conventions on the measures. We shall infer it from the exact jump formula, stated in the special case that $D^{\prime}=1$, $G=M$ and $M$ quasisplit, and with $(M, \alpha, \sigma)$ in place of $\left(\widetilde{M}^{\prime}, \alpha^{\prime}, \sigma^{\prime}\right)$. If $h \in \mathcal{C}(M, \zeta)$, Harish-Chandra's original jump formula for the invariant orbital integral

$$
h_{M}\left(\gamma \exp \theta H_{\alpha}\right), \quad \theta \neq 0,
$$


takes the form

$$
j_{\alpha}\left(\varepsilon h_{M}(\gamma)\right)=-\pi i\left\|H_{\alpha}\right\| h_{M_{\alpha}}(\gamma),
$$

expressed in terms of the obvious variant of notation above. (See [H2, Theorem 9.1], [A2, Lemma 6.3].) Shelstad's jump formula for the stable orbital integral

$$
h^{M}\left(\sigma \exp \theta H_{\alpha}\right), \quad \theta \neq 0,
$$

becomes

$$
j_{\alpha}\left(\varepsilon h^{M}(\sigma)\right)=-\pi i d_{\alpha}\left\|H_{\alpha}\right\| h^{M_{\alpha}}(\sigma),
$$

for $d_{\alpha} \in\{1,2\}$, as above. It can be derived from (3.9) in the same way that S1, Lemma 4.3] was proved on p. 30 of [S1] from Lemmas 4.5 and 4.2 there, provided of course that one takes into account the different normalizations of measures. The original constant is thus given by

$$
k_{\alpha}^{\prime}=-\pi i d_{\alpha}^{\prime}\left\|H_{\alpha}^{\prime}\right\|,
$$

where $d_{\alpha}^{\prime}=d_{\alpha^{\prime}}$ is the analogue for $\alpha^{\prime}$ of $d_{\alpha}$.

\section{$\S 4$. Stabilization of Parabolic boundary conditions}

In this section we shall consider the boundary component defined by a real root $\beta^{\prime}$ of $T^{\prime}$. We will again obtain jump conditions for the discontinuities of our distributions. In this case, it will be necessary to describe the values of the jumps quite precisely. To simplify matters, we may as well assume that $T^{\prime}$ is $\mathbb{R}$-elliptic in $M^{\prime}$.

The problem is to stabilize the corresponding discontinuities for the basic invariant distributions $I_{M}(\gamma, f)$. Suppose that $T$ is a maximal torus in $M$ over $\mathbb{R}$ for which $T^{\prime}$ is an $M$-image in $M^{\prime}$. Then $T$ is $\mathbb{R}$-elliptic in $M$. The real root $\beta^{\prime}$ corresponds to a real root $\beta$ of $(G, T)$. As in $\S 3$, we shall sometimes treat $\gamma$ as an element in $T(\mathbb{R})$ rather than an elliptic conjugacy class in $M(\mathbb{R})$. In particular, we often regard $I_{M}(\gamma, f)$ as a smooth function of $\gamma \in T_{G \text {-reg }}(\mathbb{R})$.

The discontinuities for $I_{M}(\gamma, f)$ about $\beta$ are expressed in terms of a modified distribution. Let $M_{\beta} \supset M$ be the Levi subgroup of $G$ for which

$$
\mathfrak{a}_{M_{\beta}}=\left\{H \in \mathfrak{a}_{M}: \beta(H)=0\right\} .
$$

We then set

$$
I_{M}^{\beta}(\gamma, f)=I_{M}(\gamma, f)+\left\|\beta^{\vee}\right\| \log \left|\beta(\gamma)-\beta(\gamma)^{-1}\right| I_{M_{\beta}}(\gamma, f),
$$

where $\left\|\beta^{\vee}\right\|$ is the norm of the coroot $\beta^{\vee}$, relative to the inner product on $\mathfrak{a}_{M}$ that is implicit in the definition of $I_{M}(\gamma, f)$. (It is understood that $I_{M_{\beta}}(\gamma, f)$ is defined with respect to the restriction to $\mathfrak{a}_{M_{\beta}}$ of the inner product on $\mathfrak{a}_{M}$.) This is the modified distribution. We shall review the jump conditions it satisfies about $\beta$, and then see how to stabilize them.

There is a preliminary matter to be treated first. It is to reformulate the definitions of $\S 1$ in terms of the $\beta$-modified distributions $I_{M}^{\beta}(\gamma, f)$. In proving the required compatibility conditions, we shall introduce some notions that will also be needed in our later stabilization of the boundary conditions.

The objects $M^{\prime}, T^{\prime}$ and $\beta^{\prime}$ are fixed. We again work with a given transfer factor $\Delta_{M}$, with associated data $\left(\widetilde{M}^{\prime}, \widetilde{\xi}_{M}^{\prime}\right)$ and $\widetilde{T}^{\prime}$, even though $\Delta_{M}$ is ultimately supposed to vary. A root $\beta$ of $(G, T)$ that transfers to $\beta^{\prime}$ is unique, in contrast to the case studied in the last section. This is because $\beta$ can be identified with a character on 
the split component $A_{M}$ of the center of $M$ (and hence also a character on each split component $A_{M_{\iota}}$ ). It therefore transfers under the canonical isomorphism between $A_{M}$ and $A_{M^{\prime}}$ to a character on $A_{M^{\prime}}$, which can be identified with $\beta^{\prime}$.

The coroot $\beta^{\vee}$ can be identified with a character on $\widehat{M}$. The kernel

$$
\widehat{Z}_{\beta}=\left\{z \in Z(\widehat{M})^{\Gamma}: \beta^{\vee}(z)=1\right\}
$$

of its restriction to $Z(\widehat{M})^{\Gamma}$ will be of special interest. Observe that $Z\left(\widehat{M}_{\beta}\right)^{\Gamma}$ is a subgroup of $\widehat{Z}_{\beta}$, with finite quotient

$$
\mathcal{K}_{\beta}=\widehat{Z}_{\beta} / Z\left(\widehat{M}_{\beta}\right)^{\Gamma} .
$$

We shall write $\mathcal{E}_{M^{\prime}}^{\beta}(G)$ for the subset of data $G^{\prime} \in \mathcal{E}_{M^{\prime}}(G)$ such that $\beta^{\prime}$ is a root of $\left(G^{\prime}, T^{\prime}\right)$. Recall that $\mathcal{E}_{M^{\prime}}(G)$ is parametrized by the set of points $s^{\prime}$ in $s_{M}^{\prime} Z(\widehat{M})^{\Gamma}$, taken modulo $Z(\widehat{G})^{\Gamma}$. An element $G^{\prime}$ in $\mathcal{E}_{M^{\prime}}(G)$ belongs to $\mathcal{E}_{M^{\prime}}^{\beta}(G)$ if and only if the corresponding point $s^{\prime}$ lies in

$$
\widehat{Z}_{\beta}^{+}=\left\{s^{\prime} \in s_{M}^{\prime} Z(\widehat{M})^{\Gamma}: \beta^{\vee}\left(s^{\prime}\right)=1\right\},
$$

a set on which $\widehat{Z}_{\beta}$ acts simply transitively.

Let $\sigma^{\prime}$ be a point in $\widetilde{T}_{G \text {-reg }}^{\prime}(\mathbb{R})$. We define modified forms of distributions in $\S 2$ by

$$
I_{M}^{\beta}\left(\sigma^{\prime}, f\right)=I_{M}\left(\sigma^{\prime}, f\right)+\left\|\beta^{\vee}\right\| \log \left|\beta^{\prime}\left(\sigma^{\prime}\right)-\beta^{\prime}\left(\sigma^{\prime}\right)^{-1}\right| I_{M_{\beta}}\left(\sigma^{\prime}, f\right)
$$

and

$$
I_{M}^{\mathcal{E}, \beta}\left(\sigma^{\prime}, f\right)=I_{M}^{\mathcal{E}}\left(\sigma^{\prime}, f\right)+\left\|\beta^{\vee}\right\| \log \left|\beta^{\prime}\left(\sigma^{\prime}\right)-\beta^{\prime}\left(\sigma^{\prime}\right)^{-1}\right| I_{M_{\beta}}^{\mathcal{E}}\left(\sigma^{\prime}, f\right) .
$$

If $G$ is quasisplit, we set

$$
S_{M}^{G, \beta}(\sigma, f)=S_{M}^{G}(\sigma, f)+\left|\mathcal{K}_{\beta}\right|^{-1}\left\|\beta^{\vee}\right\| \log \left|\beta(\sigma)-\beta(\sigma)^{-1}\right| S_{M_{\beta}}^{G}(\sigma, f),
$$

for any $\sigma \in T_{G \text {-reg }}(\mathbb{R})$. We also set $S_{M}^{G, \beta}\left(M^{\prime}, \sigma^{\prime}, f\right)$ equal to the original distribution $S_{M}^{G}\left(M^{\prime}, \sigma^{\prime}, f\right)$ if $M^{\prime} \neq M^{*}$, and equal to the distribution

$$
\widehat{S}_{M^{*}}^{G^{*}, \beta^{*}}\left(\sigma^{*}, f^{*}\right)=S_{M}^{G, \beta}(\sigma, f)
$$

if $M^{\prime}=M^{*}$ and $(\beta, \sigma)$ maps to the pair $\left(\beta^{*}, \sigma^{*}\right)=\left(\beta^{\prime}, \sigma^{\prime}\right)$. It follows inductively from the descent formulas (2.12)-(2.15) that the $\beta$-versions of the distributions satisfy the obvious analogue of Theorem 1.1 if and only if the original distributions satisfy the theorem itself. We will need to know that the $\beta$-distributions also satisfy analogues of the relations (1.4), (1.8), (1.9) and (1.10).

It follows from the definitions that

$$
I_{M}^{\beta}\left(\sigma^{\prime}, f\right)=\sum_{\gamma \in \Gamma_{G-\mathrm{reg}}(M)} \Delta_{M}\left(\sigma^{\prime}, \gamma\right) I_{M}^{\beta}(\gamma, f),
$$

since $\beta^{\prime}\left(\sigma^{\prime}\right)=\beta(\gamma)$ whenever $\Delta_{M}\left(\sigma^{\prime}, \gamma\right) \neq 0$. This is the analogue of (1.4). If $G$ is quasisplit, the analogue

$$
I_{M}^{\mathcal{E}, \beta}\left(\sigma^{\prime}, f\right)=I_{M}^{\beta}\left(\sigma^{\prime}, f\right)
$$

of (1.10) holds by definition. To formulate analogues of the other two relations, we define $\widehat{S}_{\tilde{M^{\prime}}}^{\tilde{G}^{\prime}, \beta^{\prime}}\left(\sigma^{\prime}, f^{\prime}\right)$ for any $G^{\prime} \in \mathcal{E}_{M^{\prime}}(G)$ by using the prescription above if $G^{\prime}$ belongs to $\mathcal{E}_{M^{\prime}}^{\beta}(G)$, and setting it equal to $\widehat{S}_{\tilde{M}^{\prime}}^{\tilde{G}^{\prime}}\left(\sigma^{\prime}, f^{\prime}\right)$ otherwise. 
Lemma 4.1. (a) If $G$ is not quasisplit,

$$
I_{M}^{\mathcal{E}, \beta}\left(\sigma^{\prime}, f\right)=\sum_{G^{\prime} \in \mathcal{E}_{M^{\prime}}(G)} \iota_{M^{\prime}}\left(G, G^{\prime}\right) \widehat{S}_{\tilde{M}^{\prime}}^{\tilde{G}^{\prime}, \beta^{\prime}}\left(\sigma^{\prime}, f^{\prime}\right) .
$$

(b) If $G$ is quasisplit,

$$
S_{M}^{G, \beta}\left(M^{\prime}, \sigma^{\prime}, f\right)=I_{M}^{\beta}\left(\sigma^{\prime}, f\right)-\sum_{G^{\prime} \in \mathcal{E}_{M^{\prime}}^{0}(G)} \iota_{M^{\prime}}\left(G, G^{\prime}\right) \widehat{S}_{\tilde{M}^{\prime}}^{\tilde{G}^{\prime}, \beta^{\prime}}\left(\sigma^{\prime}, f^{\prime}\right) .
$$

Proof. Following [A11, §3], we set $\varepsilon(G)$ equal to 1 or 0 , according to whether $G$ is quasisplit or not. The formulas (1.8)-(1.10) can then be combined as an identity between the difference

$$
I_{M}^{\mathcal{E}}\left(\sigma^{\prime}, f\right)-\varepsilon(G) S_{M}^{G}\left(M^{\prime}, \sigma^{\prime}, f\right)
$$

and the sum

$$
\sum_{G^{\prime} \in \mathcal{E}_{M^{\prime}}^{0}(G)} \iota_{M^{\prime}}\left(G, G^{\prime}\right) \widehat{S}_{\tilde{M}^{\prime}}^{\tilde{G}^{\prime}}\left(\sigma^{\prime}, f^{\prime}\right) .
$$

We need to establish a similar identity between their $\beta$-analogues

$$
I_{M}^{\mathcal{E}, \beta}\left(\sigma^{\prime}, f\right)-\varepsilon(G) S_{M}^{G, \beta}\left(M^{\prime}, \sigma^{\prime}, f\right)
$$

and

$$
\sum_{G^{\prime} \in \mathcal{E}_{M^{\prime}}^{0}(G)} \iota_{M^{\prime}}\left(G, G^{\prime}\right) \widehat{S}_{\tilde{M}^{\prime}}^{\tilde{G}^{\prime}, \beta^{\prime}}\left(\sigma^{\prime}, f^{\prime}\right) .
$$

By definition, (4.4) equals the sum of (4.2) and the product of

$$
\left\|\beta^{\vee}\right\| \log \left|\beta^{\prime}\left(\sigma^{\prime}\right)-\beta^{\prime}\left(\sigma^{\prime}\right)\right|^{-1}
$$

with

$$
\sum_{G^{\prime} \in \mathcal{E}_{M^{\prime}}^{\beta}(G) \cap \mathcal{E}_{M^{\prime}}^{0}(G)}\left|\mathcal{K}_{\beta^{\prime}}\right|^{-1} \iota_{M^{\prime}}\left(G, G^{\prime}\right) \widehat{S}_{\tilde{M}_{\beta}^{\prime}}^{\tilde{G}^{\prime}}\left(\sigma^{\prime}, f^{\prime}\right) .
$$

We have written $M_{\beta}^{\prime}$ for the Levi subgroup $M_{\beta^{\prime}}^{\prime} \in \mathcal{L}\left(M^{\prime}\right)$ of $G^{\prime}$ defined for the real root $\beta^{\prime}$ as above. We identify it with an element in $\mathcal{E}_{M^{\prime}}^{\beta}\left(M_{\beta}\right)$ by projecting the point $s^{\prime}$ in $\widehat{Z}_{\beta}^{+}$that represents $G^{\prime}$ onto its image in $\widehat{Z}_{\beta}^{+} / Z\left(\widehat{M}_{\beta}\right)^{\Gamma}$. This allows us to decompose the sum over $G^{\prime}$ in (4.6) into a double sum over $M_{\beta}^{\prime} \in \mathcal{E}_{M^{\prime}}^{\beta}\left(M_{\beta}\right)$ and $G_{\beta}^{\prime} \in \mathcal{E}_{M_{\beta}^{\prime}}^{0}(G)$. Since

$$
\iota_{M^{\prime}}\left(G, G^{\prime}\right)=\iota_{M^{\prime}}\left(M_{\beta}, M_{\beta}^{\prime}\right) \iota_{M_{\beta}^{\prime}}\left(G, G_{\beta}^{\prime}\right),
$$

the expression (4.6) becomes

$$
\sum_{M_{\beta}^{\prime} \in \mathcal{E}_{M^{\prime}}^{\beta}\left(M_{\beta}\right)}\left|\mathcal{K}_{\beta^{\prime}}\right|^{-1} \iota_{M^{\prime}}\left(M_{\beta}, M_{\beta}^{\prime}\right) \sum_{G_{\beta}^{\prime} \in \mathcal{E}_{M_{\beta}^{\prime}}^{0}(G)} \iota_{M_{\beta}^{\prime}}\left(G, G^{\prime}\right) \widehat{S}_{\bar{M}_{\beta}^{\prime}}^{\tilde{G}^{\prime}}\left(\sigma^{\prime}, f^{\prime}\right) .
$$

The sum over $G_{\beta}^{\prime}$ equals

$$
I_{M_{\beta}}^{\mathcal{E}}\left(\sigma^{\prime}, f\right)-\varepsilon(G) S_{M_{\beta}}^{G}\left(M_{\beta}^{\prime}, \sigma^{\prime}, f\right) .
$$


The coefficients in the sum over $M_{\beta}^{\prime}$ satisfy

$$
\begin{aligned}
& \left|\mathcal{K}_{\beta^{\prime}}\right|^{-1} \iota_{M^{\prime}}\left(M_{\beta}, M_{\beta}^{\prime}\right) \\
= & \left|\widehat{Z}_{\beta^{\prime}} / Z\left(\widehat{M}_{\beta}^{\prime}\right)^{\Gamma}\right|^{-1}\left|Z\left(\widehat{M}^{\prime}\right)^{\Gamma} / Z(\widehat{M})^{\Gamma}\right|\left|Z\left(\widehat{M}_{\beta}^{\prime}\right)^{\Gamma} / Z\left(\widehat{M}_{\beta}\right)^{\Gamma}\right|^{-1} \\
= & \left|\widehat{Z}_{\beta^{\prime}} / Z\left(\widehat{M}_{\beta}\right)^{\Gamma}\right|^{-1}\left|Z\left(\widehat{M}^{\prime}\right)^{\Gamma} / Z(\widehat{M})^{\Gamma}\right| \\
= & \left|\widehat{Z}_{\beta} / Z\left(\widehat{M}_{\beta}\right)^{\Gamma}\right|^{-1} \\
= & \left|\mathcal{K}_{\beta}\right|^{-1}
\end{aligned}
$$

since every class in $Z\left(\widehat{M}^{\prime}\right)^{\Gamma} / Z(\widehat{M})^{\Gamma}$ has a representative in the kernel $\widehat{Z}_{\beta^{\prime}}$ of $\left(\beta^{\prime}\right)^{\vee}$ in $Z\left(\widehat{M}^{\prime}\right)^{\Gamma}$. (This last assertion follows in turn from the fact that $\left(\beta^{\prime}\right)^{\vee}$ maps the complex torus $\left(Z(\widehat{M})^{\Gamma}\right)^{0}$ onto $\mathbb{C}^{*}$.) The expression (4.6) therefore equals the difference

$$
\left|\mathcal{K}_{\beta}\right|^{-1} \sum_{M_{\beta}^{\prime}} I_{M_{\beta}}^{\mathcal{E}}\left(\sigma^{\prime}, f\right)-\left|\mathcal{K}_{\beta}\right|^{-1} \sum_{M_{\beta}^{\prime}} \varepsilon(G) S_{M_{\beta}}^{G}\left(M_{\beta}^{\prime}, \sigma^{\prime}, f\right) .
$$

From the descent formula (2.13) (with $M_{\beta}$ in place of $M$ ), we see that $I_{M_{\beta}}^{\mathcal{E}}\left(\sigma^{\prime}, f\right)$ is independent of $M_{\beta}^{\prime}$. Since $M_{\beta}^{\prime}$ ranges over a set on which $\mathcal{K}_{\beta}$ acts simply transitively, the first term reduces to $I_{M_{\beta}}^{\mathcal{E}}\left(\sigma^{\prime}, f\right)$. By the descent formula (2.15) (applied again to $\left.M_{\beta}\right)$, the distribution $\widehat{S}_{M_{\beta}}^{G}\left(M_{\beta}^{\prime}, \sigma^{\prime}, f\right)$ vanishes unless $M_{\beta}^{\prime}$ equals $M_{\beta}^{*}$. If $M_{\beta}^{\prime}$ does equal $M_{\beta}^{*}, M^{\prime}$ equals $M^{*}$, and the distribution equals $S_{M_{\beta}}^{G}(\sigma, f)$, for a point $\sigma$ that maps to $\sigma^{\prime}=\sigma^{*}$. It follows that (4.6) equals

$$
I_{M_{\beta}}^{\mathcal{E}}\left(\sigma^{\prime}, f\right)-\left|\mathcal{K}_{\beta}\right|^{-1} \varepsilon\left(G, M^{\prime}\right) S_{M_{\beta}}^{G}(\sigma, f),
$$

where

$$
\varepsilon\left(G, M^{\prime}\right)= \begin{cases}1, & \text { if } G \text { is quasisplit and } M^{\prime}=M^{*}, \\ 0, & \text { otherwise. }\end{cases}
$$

We can now add (4.1) to the product of (4.5) with the expression we have obtained for (4.6). The resulting expression equals (4.3), according to the definitions above. Since (4.1) equals (4.2), we conclude that (4.3) does indeed equal (4.4).

If $G$ is not quasisplit, $\varepsilon(G)=0$ and $\mathcal{E}_{M^{\prime}}^{0}(G)=\mathcal{E}_{M^{\prime}}(G)$. The equality of (4.3) and (4.4) then reduces to the required formula of (a). If $G$ is quasisplit, $\varepsilon(G)=1$ and $I_{M}^{\mathcal{E}, \beta}\left(\sigma^{\prime}, f\right)$ equals $I_{M}^{\beta}\left(\sigma^{\prime}, f\right)$. In this case, the equality of (4.3) and (4.4) becomes the required formula of (b).

We now recall the jump conditions about $\beta$ satisfied by $I_{M}^{\beta}(\gamma, f)$. Following $\S 3$, we change notation slightly, now taking $\gamma$ to be a point in general position in the subgroup

$$
T^{\beta}(\mathbb{R})=\{\gamma \in T(\mathbb{R}): \beta(\gamma)=1\}
$$

of $T(\mathbb{R})$. The centralizer $G_{\gamma}$ is a connected reductive group over $\mathbb{R}$, whose derived group $G_{\gamma, \text { der }}$ is isomorphic over $\mathbb{R}$ to either $S L(2)$ or $P G L(2)$. Let $T_{\beta}$ be an elliptic maximal torus in $G_{\gamma}$ such that the Lie algebras of $T_{\beta} \cap G_{\gamma \text {,der }}$ and $T \cap G_{\gamma \text {,der }}$ are orthogonal with respect to the Killing form. Then $T_{\beta}$ is $\mathbb{R}$-elliptic in the Levi subgroup $M_{\beta}$. This takes us back to the setting of $\S 3$, with $M, T, M_{\beta}$ and $T_{\beta}$ here in place of the groups denoted $M_{\alpha}, T_{\alpha}, M$ and $T$ in $\S 3$. In fact, if we fix an inverse Cayley transform

$$
C_{\beta}=\operatorname{Int}\left(s_{\beta}\right), \quad s_{\beta} \in G_{\gamma, \mathrm{der}},
$$


that takes $T$ to $T_{\beta}$, and let $\alpha$ be the root of $\left(M_{\beta}, T_{\beta}\right)$ corresponding to $\beta$, we have $\left(M_{\beta}\right)_{\alpha}=M,\left(T_{\beta}\right)_{\alpha}=T$ and $C_{\alpha}=C_{\beta}^{-1}$. In particular, we have the elements $H_{\beta}=Z_{\alpha}$ and $Z_{\beta}=H_{\alpha}$ in the Lie algebra of $G_{\gamma, \text { der }}$ defined in $\S 3$.

Suppose that $D$ is an invariant differential operator on $C^{\infty}(T, \zeta)$. If $w_{\beta}$ is the reflection in $T$ about $\beta$, the composition

$$
w_{\beta} D=w_{\beta} \circ D \circ w_{\beta}^{-1}
$$

is also an invariant differential operator on $C^{\infty}(T, \zeta)$, and the transform

$$
D_{\beta}=D_{\beta, C_{\beta}}=C_{\beta}\left(w_{\beta} D-D\right)
$$

is an invariant differential operator on $C^{\infty}\left(T_{\beta}, \zeta\right)$. We write $j_{\beta}\left(D I_{M}^{\beta}(\gamma, f)\right)$ for the jump

$$
\lim _{r \rightarrow 0+} D I_{M}^{\beta}\left(\gamma \exp r H_{\beta}, f\right)-\lim _{r \rightarrow 0-} D I_{M}^{\beta}\left(\gamma \exp r H_{\beta}, f\right) .
$$

The jump condition for $I_{M}^{\beta}(\gamma, f)$ is the identity

$$
j_{\beta}\left(D I_{M}^{\beta}(\gamma, f)\right)=\lim _{\theta \rightarrow 0} \varepsilon(\theta) D_{\beta} I_{M_{\beta}}\left(\gamma_{\beta}(\theta), f\right),
$$

for

$$
\varepsilon(\theta)=-i \cdot \operatorname{sign} \theta
$$

as in (3.8), and

$$
\gamma_{\beta}(\theta)=\gamma_{\beta}\left(C_{\beta}, \theta\right)=\gamma \exp \left(\theta Z_{\beta}\right) .
$$

It of course includes the existence of the two half limits on the left hand side of (4.7). The existence of the limit on the right hand side follows from the fact that $D_{\beta}$ is antisymmetric with respect to reflection about $\alpha$ in $T_{\beta}$. (See A2, Theorem 6.1 and Corollary 8.4] and [A3, Lemma 13.1]. The factor $\varepsilon(\theta)$ was inadvertently omitted from the second reference.)

Our task is to stabilize (4.7). The starting point will be a fixed element $\sigma^{\prime}$ in general position in the kernel $(\widetilde{T})^{\beta^{\prime}}(\mathbb{R})$ of $\beta^{\prime}$ in $\widetilde{T}^{\prime}(\mathbb{R})$, and an invariant differential operator $D^{\prime}$ on $C^{\infty}\left(T^{\prime}, M, \zeta\right)$. We note that $\sigma^{\prime}$ is still strongly $M$-regular, even though it is not $G$-regular, since $\beta$ is not a root of $(M, T)$. The transfer $H_{\beta}^{\prime}$ of $H_{\beta}$ under any $M$-admissible isomorphism from $T$ to $T^{\prime}$ depends only on $\beta^{\prime}$. We write

$$
j_{\beta^{\prime}}\left(a^{\prime}\left(\sigma^{\prime}\right)\right)=\lim _{r \rightarrow 0+} a^{\prime}\left(\sigma^{\prime} \exp r H_{\beta}^{\prime}\right)-\lim _{r \rightarrow 0-} a^{\prime}\left(\sigma^{\prime} \exp r H_{\beta}^{\prime}\right),
$$

for any section $a^{\prime} \in C^{\infty}\left(T_{G \text {-reg }}^{\prime}, M, \zeta\right)$ for which the two half limits exist.

Our main concern will be the jump attached to the section

$$
a^{\prime}(\cdot)=D^{\prime} I_{M}^{\beta}(\cdot, f)
$$

According to the definition (1.4), we can express this jump as a sum over classes $\gamma \in \Gamma_{\text {reg }}(M)$ with $\Delta_{M}\left(\sigma^{\prime}, \gamma\right) \neq 0$. Writing $\gamma$ also for a fixed representative in $M(\mathbb{R})$ of a given class, we obtain a unique $M$-admissible isomorphism from the maximal torus $T=M_{\gamma}$ to $T^{\prime}$ that takes $\gamma$ to the image of $\sigma^{\prime}$ in $T^{\prime}$. We use it to transfer $D^{\prime}$ to an invariant differential operator $D$ on $C^{\infty}(T, \zeta)$. It then follows from (1.4) and (2.4) (or rather a variant of (2.4) for operators that are not $W_{\mathbb{R}}(M, T)$-symmetric) that

$$
j_{\beta^{\prime}}\left(D^{\prime} I_{M}^{\beta}\left(\sigma^{\prime}, f\right)\right)=\sum_{\gamma \in \Gamma_{\mathrm{reg}}(M)} \Delta_{M}\left(\sigma^{\prime}, \gamma\right) j_{\beta}\left(D I_{M}^{\beta}(\gamma, f)\right) .
$$


The formula (4.7) allows us to express the right hand side of (4.8) as a limit

$$
\lim _{\theta \rightarrow 0} \varepsilon(\theta) \sum_{\gamma \in \Gamma_{\mathrm{reg}}(M)} \Delta_{M}\left(\sigma^{\prime}, \gamma\right) D_{\beta} I_{M_{\beta}}\left(\gamma_{\beta}(\theta), f\right),
$$

in which the differential operator $D_{\beta}$ on the right depends on $D^{\prime}, \gamma$ and $C_{\beta}$. We would like to express this limit in terms of endoscopic data $M_{\beta}^{\prime}$ for $M_{\beta}$. The basic problem is to inflate the sum over $\Gamma_{\text {reg }}(M)$ to one over $\Gamma_{\text {reg }}\left(M_{\beta}\right)$.

The right hand side of (4.8) amounts to a sum over the finite subset of elements $\gamma \in \Gamma_{\text {reg }}(M)$ with $\Delta_{M}\left(\sigma^{\prime}, \gamma\right) \neq 0$. We fix one such element $\gamma$. The sum can then be taken over the set of $\gamma_{1} \in \Gamma_{\text {reg }}(M)$ in the stable class of $\gamma$. There is a bijection

$$
\gamma_{1} \longrightarrow \operatorname{inv}\left(\gamma, \gamma_{1}\right)
$$

from this set onto the group

$$
\mathcal{E}(T)=\operatorname{Im}\left(H^{1}\left(\mathbb{R}, T_{\mathrm{sc}}\right) \longrightarrow H^{1}(\mathbb{R}, T)\right),
$$

whose definition we recall.

Since $M$ is supposed to be a $K$-group in its own right, it comes with an isomorphism $\psi_{\iota \iota_{1}}$ from the component $M_{\iota_{1}}$ of $\gamma_{1}$ onto the component $M_{\iota}$ of $\gamma$. The image $\psi_{\iota \iota_{1}}\left(\gamma_{1}\right)$ of $\gamma_{1}$ is $M_{\iota}$-conjugate to $\gamma$. We can therefore write

$$
\gamma=h \psi_{\iota \iota_{1}}\left(\gamma_{1}\right) h^{-1},
$$

for some point $h \in M_{\iota, \mathrm{sc}}(\mathbb{C})$. If $\tau$ belongs to the Galois group $\Gamma=\operatorname{Gal}(\mathbb{C} / \mathbb{R})$, we have

$$
\begin{aligned}
\gamma=\tau(\gamma) & =\tau(h) \tau\left(\psi_{\iota \iota_{1}}\left(\gamma_{1}\right)\right) \tau\left(h^{-1}\right) \\
& =\tau(h)\left(\tau\left(\psi_{\iota \iota_{1}}\right)\right)\left(\gamma_{1}\right) \tau\left(h^{-1}\right),
\end{aligned}
$$

since $\gamma$ and $\gamma_{1}$ are both defined over $\mathbb{R}$. It follows that

$$
\gamma_{1}=\left(\tau\left(\psi_{\iota \iota_{1}}\right)^{-1} \circ \operatorname{Int}(\tau(h))^{-1}\right)(\gamma),
$$

and consequently that

$$
\begin{aligned}
\gamma & =\left(\operatorname{Int}(h) \circ \psi_{\iota \iota_{1}}\right)\left(\gamma_{1}\right) \\
& =\left(\operatorname{Int}(h) \circ \psi_{\iota \iota_{1}} \circ \tau\left(\psi_{\iota \iota_{1}}\right)^{-1} \circ \operatorname{Int}(\tau(h))^{-1}\right)(\gamma) \\
& =\operatorname{Int}\left(h u_{\iota \iota_{1}}(\tau) \tau(h)^{-1}\right)(\gamma),
\end{aligned}
$$

in view of condition (i) on [A11, p. 212]. Since $\gamma$ is strongly $M$-regular, the function

$$
\tau \longrightarrow h u_{\iota \iota_{1}}(\tau) \tau(h)^{-1}, \quad \tau \in \Gamma,
$$

takes values in the preimage $T_{\mathrm{sc}}$ of $T$ in $M_{\iota, \mathrm{sc}}$. This function is a 1-cocycle, by virtue of the fact that $M$ is a $K$-group. We define $\operatorname{inv}\left(\gamma, \gamma_{1}\right)$ to be its image in $H^{1}(\mathbb{R}, T)$. It is then easy to check that the mapping (4.9) is a bijection from the original set of conjugacy classes onto the group $\mathcal{E}(T)$. (The surjectivity of the mapping also relies on the fact that $G$ is a $K$-group.)

We recall that by Tate-Nakayama duality, there is a canonical isomorphism from $\mathcal{E}(T)$ onto the dual of the finite abelian group

$$
\mathcal{K}(T)=\pi_{0}\left(\widehat{T}^{\Gamma} / Z(\widehat{G})^{\Gamma}\right)=\widehat{T}^{\Gamma} / Z(\widehat{M})^{\Gamma} .
$$

(See for example [K2]. The second equality is a consequence of the fact that $T$ is elliptic in $M$. It is easy to see that $\mathcal{K}(T)$ is in fact a 2-group.) Keep in mind that there is a unique admissible isomorphism from $T=M_{\gamma}$ onto $T^{\prime}$ that takes $\gamma$ to 
the image in $T^{\prime}(\mathbb{R})$ of $\sigma^{\prime}$. We write $\kappa_{M}^{\prime}=\kappa\left(\sigma^{\prime}, \gamma\right)$ for the projection onto $\mathcal{K}(T)$ of the image of $s_{M}^{\prime}$ in $\widehat{T}^{\Gamma}$ under the dual isomorphism from $\widehat{T}^{\prime}$ to $\widehat{T}$. We recall here that $s_{M}^{\prime}$ denotes the element in the subgroup $Z\left(\widehat{M}^{\prime}\right)^{\Gamma}$ of $\left(\widehat{T}^{\prime}\right)^{\Gamma}$ attached to $M^{\prime}$. The transfer factors in (4.8) then satisfy an identity

$$
\Delta_{M}\left(\sigma^{\prime}, \gamma\right)=\Delta_{M}\left(\sigma^{\prime}, \gamma_{1}\right)\left\langle\kappa_{M}^{\prime}, \operatorname{inv}\left(\gamma, \gamma_{1}\right)\right\rangle .
$$

(See [A11, p. 224] or [KS, Lemma 5.1.D]. The usual custom [LS1] is to take $T$ to be a maximal torus in the quasisplit inner twist $G^{*}$ of $G$ that we have generally suppressed here. The preimage of $\kappa_{M}^{\prime}$ in $\widehat{T}^{\Gamma}$, denoted by $\left(s_{M}^{\prime}\right)_{T}$ in [LS1], then depends on a choice of admissible embedding of $T^{\prime}$ into $G^{*}$ with image $T$.)

We need to relate both $\mathcal{E}(T)$ and $\mathcal{K}(T)$ with the corresponding groups $\mathcal{E}\left(T_{\beta}\right)$ and $\mathcal{K}\left(T_{\beta}\right)$ attached to $T_{\beta}$. Our discussion at this point is motivated by that of [S1, $\S 4$. The inverse Cayley transform $C_{\beta}=\operatorname{Int}\left(s_{\beta}\right)$ is an isomorphism from $T$ to $T_{\beta}$. We use it to identify the dual group $\widehat{T}_{\beta}$ with $\widehat{T}$. Then

$$
\mathcal{K}\left(T_{\beta}\right)=\pi_{0}\left(\widehat{T}^{\Gamma_{\beta}} / Z(\widehat{G})^{\Gamma_{\beta}}\right)=\widehat{T}^{\Gamma_{\beta}} / Z\left(\widehat{M}_{\beta}\right)^{\Gamma_{\beta}},
$$

where $\Gamma_{\beta}=\left\{1, \sigma_{T_{\beta}}\right\}$ represents the action of the Galois group $\Gamma$ on $\widehat{T}$ obtained from $T_{\beta}$. The nontrivial operator in $\Gamma_{\beta}$ satisfies

$$
\sigma_{T_{\beta}}=\sigma_{T} w_{\beta}^{\vee}
$$

where $\sigma_{T}$ is the corresponding operator on $\widehat{T}$ obtained from $T$, and $w_{\beta}^{\vee}=w_{\beta^{\vee}}$ is the simple reflection in $\widehat{T}$ about $\beta^{\vee}$. In particular,

$$
Z\left(\widehat{M}_{\beta}\right)^{\Gamma_{\beta}}=Z\left(\widehat{M}_{\beta}\right)^{\Gamma},
$$

since $w_{\beta}^{\vee}$ centralizes $Z\left(\widehat{M}_{\beta}\right)$. Following standard notation, we write

$$
w_{\beta}^{\vee}(t)=t\left(\beta^{\vee}(t)\right)^{-\beta}, \quad t \in \widehat{T} .
$$

The group $\widehat{Z}_{\beta}$, defined prior to Lemma 4.1 as the kernel of $\beta^{\vee}$ in $Z(\widehat{M})^{\Gamma}$, is therefore contained in $\widehat{T}^{\Gamma_{\beta}}$. Its quotient

$$
\mathcal{K}_{\beta}=\widehat{Z}_{\beta} / Z\left(\widehat{M}_{\beta}\right)^{\Gamma}=\widehat{Z}_{\beta} / Z\left(\widehat{M}_{\beta}\right)^{\Gamma_{\beta}}
$$

is a subgroup of $\mathcal{K}\left(T_{\beta}\right)$, whose associated quotient in $\mathcal{K}\left(T_{\beta}\right)$ we denote by

$$
\mathcal{K}_{\beta}\left(T_{\beta}\right)=\mathcal{K}\left(T_{\beta}\right) / \mathcal{K}_{\beta}
$$

As a co-root for $\widehat{G}, \beta$ represents a mapping of $G L(1, \mathbb{C})$ into $\widehat{T}$ whose image is contained in $Z(\widehat{M})^{\Gamma}$, since $\beta$ is real and $T$ is $\mathbb{R}$-elliptic in $M$. Any point $t \in \widehat{T}^{\Gamma}$ therefore has a $Z(\widehat{M})^{\Gamma}$-translate

$$
t_{\beta}=t z, \quad z \in Z(\widehat{M})^{\Gamma},
$$

with $\beta^{\vee}\left(t_{\beta}\right)=1$, and which consequently lies in $\widehat{T}^{\Gamma_{\beta}}$. Since $z$ is uniquely determined modulo $\widehat{Z}_{\beta}$, the correspondence $t \rightarrow t_{\beta}$ gives a well defined injection from $\mathcal{K}(T)$ into $\mathcal{K}_{\beta}\left(T_{\beta}\right)$. Since $\beta^{\vee}$ is trivial on $\widehat{Z}_{\beta}$, it descends to a character on the quotient $\mathcal{K}_{\beta}\left(T_{\beta}\right)$ of $\mathcal{K}\left(T_{\beta}\right)$. The image of the injection is then the kernel

$$
\mathcal{K}_{\beta}(T)=\left\{t \in \mathcal{K}_{\beta}\left(T_{\beta}\right): \beta^{\vee}(t)=1\right\}
$$

of $\beta^{\vee}$ in $\mathcal{K}_{\beta}\left(T_{\beta}\right)$. 
We have constructed a diagram

$$
\begin{array}{rlr}
\mathcal{K}\left(T_{\beta}\right) \rightarrow \mathcal{K}_{\beta}\left(T_{\beta}\right) & \hookleftarrow & \mathcal{K}_{\beta}(T) \\
& & \uparrow ? \\
& \mathcal{K}(T)
\end{array}
$$

of homomorphisms of (abelian) 2-groups. This in turn is dual to a diagram

$$
\begin{array}{rlc}
\mathcal{E}\left(T_{\beta}\right) \hookleftarrow \quad \mathcal{E}_{\beta}\left(T_{\beta}\right) \rightarrow & \mathcal{E}_{\beta}(T) \\
& & \underset{\mid l}{ } \\
& \mathcal{E}(T)
\end{array}
$$

of homomorphisms among corresponding dual groups. The group $\mathcal{E}_{\beta}\left(T_{\beta}\right)$ is the annihilator of $\mathcal{K}_{\beta}$ in $\mathcal{E}\left(T_{\beta}\right)$. As a character on $\mathcal{K}_{\beta}\left(T_{\beta}\right)$, of order $d_{\beta}$ equal to 1 or 2 , $\beta^{\vee}$ generates a subgroup of $\mathcal{E}_{\beta}\left(T_{\beta}\right)$ of order $d_{\beta}$. The group $\mathcal{E}_{\beta}(T)$ is the associated quotient. (Notice that by regarding $\beta^{\vee}$ as an element in $\mathcal{E}_{\beta}\left(T_{\beta}\right)$, we are identifying it with the coroot $\alpha^{\vee}$. This is of course a consequence of our having identified $\widehat{T}_{\beta}$ with $\widehat{T}$.)

The mappings in (4.11) were defined in terms of the inverse Cayley transform $C_{\beta}$. Now $C_{\beta}$ is determined by $T$ and $T_{\beta}$ only up to the action of $\Gamma$. If $C_{\beta}$ is replaced by its complex conjugate

$$
\bar{C}_{\beta}=C_{\beta} w_{\beta}=w_{\alpha} C_{\beta},
$$

the isomorphism of $T$ with $T_{\beta}$, which was attached to $C_{\beta}$ and allowed us to identify $\widehat{T}_{\beta}$ with $\widehat{T}$, has to be composed with $w_{\beta}$. However, it follows from the definitions that the vertical mappings on the right hand sides of (4.11) and (4.12) do not change, and are therefore independent of $C_{\beta}$. This is essentially equivalent to the identity

$$
\operatorname{inv}\left(\gamma_{\beta}, w_{\alpha}\left(\gamma_{\beta}\right)\right)=\beta^{\vee}
$$

of elements in $\mathcal{E}\left(T_{\beta}\right)$ attached to any $G$-regular element $\gamma_{\beta}$ in $T_{\beta}(\mathbb{R})$. (See also [S2, Proposition 2.1].) The identity (4.13) also implies that the order $d_{\beta}$ equals the integer $d_{\alpha}$ of $\S 3$.

Suppose now that $M_{\beta}^{\prime}$ belongs to $\mathcal{E}_{M^{\prime}}^{\beta}\left(M_{\beta}\right)$. Then $\beta^{\prime}$ is a real root of $\left(M_{\beta}^{\prime}, T^{\prime}\right)$. It provides the setting for an inverse Cayley transform $C_{\beta}^{\prime}=C_{\beta^{\prime}}^{\prime}$ from $T^{\prime}$ to an elliptic maximal torus $T_{\beta}^{\prime}=T_{\beta^{\prime}}^{\prime}$ in $M_{\beta}^{\prime}$. This gives us an element $Z_{\beta}^{\prime}=Z_{\beta^{\prime}}$ in the Lie algebra of $T_{\beta}^{\prime}(\mathbb{R})$, and a strongly $G$-regular point

$$
\sigma_{\beta}^{\prime}(\theta)=\sigma^{\prime} \exp \left(\theta Z_{\beta}^{\prime}\right)
$$

in $\widetilde{T}_{\beta}^{\prime}(\mathbb{R})$ for each small real number $\theta \neq 0$. It also attaches an invariant differential operator

$$
D_{\beta}^{\prime}=D_{\beta^{\prime}}^{\prime}=C_{\beta}^{\prime}\left(w_{\beta}^{\prime} D^{\prime}-D^{\prime}\right)=C_{\beta^{\prime}}\left(w_{\beta^{\prime}} D^{\prime}-D^{\prime}\right)
$$

on $C^{\infty}\left(T_{\beta}^{\prime}, M_{\beta}, \zeta\right)$ to the original differential operator $D^{\prime}$. One sees easily from the definitions that $D_{\beta}^{\prime}$ is the transfer of the differential operator $D_{\beta}$, relative to the admissible isomorphism from $T_{\beta}$ to $T_{\beta}^{\prime}$ that takes $\gamma_{\beta}(\theta)$ to $\sigma_{\beta}^{\prime}(\theta)$. In other words, it fits into the commutative diagram

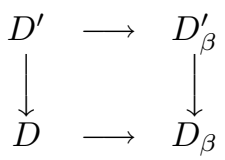


Lemma 4.2. Suppose that $\Delta_{M}$ is the restriction of a transfer factor $\Delta_{M_{\beta}} \in \mathcal{T}\left(M_{\beta}^{\prime}, M_{\beta}\right)$ to $M$. Then

$$
\Delta_{M}\left(\sigma^{\prime}, \gamma\right)=\Delta_{M_{\beta}}\left(\sigma_{\beta}^{\prime}(\theta), \gamma_{\beta}(\theta)\right),
$$

for any $\gamma \in \Gamma_{\text {reg }}(M)$ and any small real number $\theta \neq 0$.

Proof. This lemma is implicit in the work of Shelstad, specifically the transfer of elliptic boundary conditions that was part of her proof of the transfer of functions. The proof was actually carried out before the introduction of general transfer factors, but was later shown to be compatible with the transfer factors LS2, Theorem 2.6.A]. Rather than attempt to relate Shelstad's original arguments S1 to the later transfer factors of [LS1], we shall work backwards. We shall deduce the lemma from the existence of the general transfer mapping for $M_{\beta}$.

We can assume that $\sigma^{\prime}$ is an $M$-image of $\gamma$, since both sides of the putative formula would otherwise vanish. As a nonvanishing function on a domain in the product of $\widetilde{T}_{\beta, G \text {-reg }}^{\prime}(\mathbb{R})$ with $T_{\beta, G \text {-reg }}(\mathbb{R})$, the transfer factor $\Delta_{M_{\beta}}(\cdot, \cdot)$ is the product of a locally constant function with a character on $\tilde{M}_{\beta}^{\prime}(\mathbb{R})$. It follows that the function

$$
\Delta_{M_{\beta}}=\Delta_{M_{\beta}}\left(\sigma_{\beta}^{\prime}(\theta), \gamma_{\beta}(\theta)\right), \quad \theta>0,
$$

of $\theta$ is actually constant. In fact, it follows from (4.10), (4.13) and the fact that $\sigma_{\beta}^{\prime}(\theta)$ is stably conjugate to $\sigma_{\beta}^{\prime}(-\theta)$ that

$$
\Delta_{M_{\beta}}=\Delta_{M_{\beta}}\left(\sigma_{\beta}^{\prime}(\theta), \gamma_{\beta}(\theta)\right)=\Delta_{M_{\beta}}\left(\sigma_{\beta}^{\prime}(\theta), \gamma_{\beta}(-\theta)\right), \quad \theta \neq 0 .
$$

Our task is to show that $\Delta_{M_{\beta}}$ equals $\Delta_{M}\left(\sigma^{\prime}, \gamma\right)$.

We shall compare the explicit jump conditions for invariant and stable orbital integrals discussed at the end of the last section. Recall that these conditions are formulated in terms of the imaginary noncompact root $\alpha$ of $\left(M_{\beta}, T_{\beta}\right)$ attached to $\beta$ and $C_{\beta}$, and the corresponding integer $d_{\alpha}=d_{\beta}$. These objects of course have analogues $\alpha^{\prime}$ and $d_{\alpha}^{\prime}=d_{\alpha^{\prime}}$ for $\widetilde{M}_{\beta}^{\prime}$.

Suppose that $h$ is any function in $\mathcal{C}\left(M_{\beta}, \zeta\right)$. We then have the formula

$$
j_{\alpha^{\prime}}\left(\varepsilon h^{\prime}\left(\sigma^{\prime}\right)\right)=-\pi i d_{\alpha}^{\prime}\left\|H_{\alpha}^{\prime}\right\| h_{M}^{\prime}\left(\sigma^{\prime}\right),
$$

for the jump

$$
\begin{aligned}
j_{\alpha^{\prime}}\left(\varepsilon h^{\prime}\left(\sigma^{\prime}\right)\right) & =\lim _{\theta \rightarrow 0+}\left(\varepsilon(\theta) h^{\prime}\left(\sigma_{\beta}^{\prime}(\theta)\right)-\varepsilon(-\theta) h^{\prime}\left(\sigma_{\beta}^{\prime}(-\theta)\right)\right) \\
& =\lim _{\theta \rightarrow 0+}(-i)\left(h^{\prime}\left(\sigma_{\beta}^{\prime}(\theta)\right)+h^{\prime}\left(\sigma_{\beta}^{\prime}(-\theta)\right)\right),
\end{aligned}
$$

given by (3.10). Suppose further that $h$ is a nonnegative function with $h(\gamma) \neq 0$, which is supported on a small neighbourhood of $\gamma$. The right hand side of (4.16) then reduces to

$$
-\pi i d_{\alpha}^{\prime}\left\|H_{\alpha}^{\prime}\right\| \Delta_{M}\left(\sigma^{\prime}, \gamma\right) h_{M}(\gamma)
$$

Moreover, if $\theta \neq 0$ is small, the points $\gamma_{\beta}(\theta)$ and $\gamma_{\beta}(-\theta)$ represent the only classes in $\Gamma_{G \text {-reg }}\left(M_{\beta}\right)$ in the support of $h_{M_{\beta}}$ of which $\sigma_{\beta}^{\prime}(\theta)$ is an image. We recall that $d_{\alpha}$ equals 1 or 2 , according to whether or not these points represent the same class. Therefore

$$
h^{\prime}\left(\sigma_{\beta}^{\prime}(\theta)\right)= \begin{cases}\Delta_{M_{\beta}} h_{M_{\beta}}\left(\gamma_{\beta}(\theta)\right), & \text { if } d_{\alpha}=1, \\ \Delta_{M_{\beta}}\left(h_{M_{\beta}}\left(\gamma_{\beta}(\theta)\right)+h_{M_{\beta}}\left(\gamma_{\beta}(-\theta)\right)\right), & \text { if } d_{\alpha}=2 .\end{cases}
$$


The left hand side of (4.16) consequently equals

$$
\begin{aligned}
& \lim _{\theta \rightarrow 0+}(-i) d_{\alpha} \Delta_{M_{\beta}}\left(h_{M_{\beta}}\left(\gamma_{\beta}(\theta)+h_{M_{\beta}}\left(\gamma_{\beta}(-\theta)\right)\right)\right. \\
& =\lim _{\theta \rightarrow 0+} d_{\alpha} \Delta_{M_{\beta}}\left(\varepsilon(\theta) h_{M_{\beta}}\left(\gamma_{\beta}(\theta)\right)-\varepsilon(-\theta) h_{M_{\beta}}\left(\gamma_{\beta}(-\theta)\right)\right) \\
& =d_{\alpha} \Delta_{M_{\beta}} j_{\alpha}\left(\varepsilon h_{M_{\beta}}(\gamma)\right) \\
& =-\pi i d_{\alpha}\left\|H_{\alpha}\right\| \Delta_{M_{\beta}} h_{M}(\gamma),
\end{aligned}
$$

by (3.9). The norms $\left\|H_{\alpha}^{\prime}\right\|$ and $\left\|H_{\alpha}\right\|$ are equal, since it is understood that the underlying inner products on the spaces $\mathfrak{a}_{M}^{G}$ and $\mathfrak{a}_{\tilde{M}^{\prime}}^{\tilde{G}^{\prime}}$ match. Moreover, $d_{\alpha}^{\prime}$ equals $d_{\alpha}$. This is a consequence of the properties of the diagram (4.11) and the identity $d_{\alpha}=d_{\beta}$, or alternatively, Proposition 4.4 of [S1. Since $h_{M}(\gamma) \neq 0$, the required identity

$$
\Delta_{M_{\beta}}=\Delta_{M}\left(\sigma^{\prime}, \gamma\right)
$$

then follows from (4.16).

Corollary 4.3. If $\gamma$ and $\gamma_{1}$ are as in (4.9), inv $\left(\gamma_{\beta}(\theta), \gamma_{1, \beta}(\theta)\right)$ belongs to the subgroup $\mathcal{E}_{\beta}\left(T_{\beta}\right)$ of $\mathcal{E}\left(T_{\beta}\right)$, and its image in $\mathcal{E}(T)$ under the mappings of (4.12) equals $\operatorname{inv}\left(\gamma, \gamma_{1}\right)$.

Proof. If $\kappa_{M}^{\prime}=\kappa\left(\sigma^{\prime}, \gamma\right)$ and $\kappa_{\beta}^{\prime}=\kappa_{M_{\beta}}^{\prime}=\kappa\left(\sigma_{\beta}^{\prime}(\theta), \gamma_{\beta}(\theta)\right)$, we can write

$$
\begin{aligned}
& \left\langle\kappa_{\beta}^{\prime}, \operatorname{inv}\left(\gamma_{\beta}(\theta), \gamma_{1, \beta}(\theta)\right)\right\rangle \\
& =\Delta_{M_{\beta}}\left(\sigma_{\beta}^{\prime}(\theta), \gamma_{1, \beta}(\theta)\right)^{-1} \Delta_{M_{\beta}}\left(\sigma_{\beta}^{\prime}(\theta), \gamma_{\beta}(\theta)\right) \\
& =\Delta_{M}\left(\sigma^{\prime}, \gamma_{1}\right)^{-1} \Delta_{M}\left(\sigma^{\prime}, \gamma\right) \\
& =\left\langle\kappa_{M}^{\prime}, \operatorname{inv}\left(\gamma, \gamma_{1}\right)\right\rangle,
\end{aligned}
$$

by the lemma and (4.10). If $M_{\beta}^{\prime}$ ranges over groups in $\mathcal{E}_{M^{\prime}}^{\beta}\left(M_{\beta}\right), \kappa_{\beta}^{\prime}$ ranges over the preimage in $\mathcal{K}\left(T_{\beta}\right)$ of the image of $\kappa_{M}^{\prime}$ in $\mathcal{K}_{\beta}\left(T_{\beta}\right)$ under the mappings (4.11). It follows from the last identity that inv $\left(\gamma_{\beta}(\theta), \gamma_{1, \beta}(\theta)\right)$ belongs to $\mathcal{E}_{\beta}\left(T_{\beta}\right)$. The endoscopic datum $M^{\prime}$ is supposed to be fixed. However, we can still let it vary here in order to establish the corollary. Since $\kappa_{M}^{\prime}$ will then vary over $\mathcal{K}(T)$, the second assertion of the corollary also follows from the identity.

We can now apply what we have learned to the jump formula (4.8). It follows from (4.7) and Lemma 4.2 that the right hand side of (4.8) equals the limit

$$
\lim _{\theta \rightarrow 0} \varepsilon(\theta) \sum_{\gamma \in \Gamma_{\mathrm{reg}}(M)} \Delta_{M_{\beta}}\left(\sigma_{\beta}^{\prime}(\theta), \gamma_{\beta}(\theta)\right) D_{\beta} I_{M_{\beta}}\left(\gamma_{\beta}(\theta), f\right) .
$$

Let $\Gamma_{\beta}\left(M_{\beta}, \theta\right)$ be the set of classes in $\Gamma_{G \text {-reg }}\left(M_{\beta}\right)$ that lie in the stable class of $\gamma_{\beta}(\theta)$, for some $\gamma \in \Gamma_{\text {reg }}(M)$ in the stable class attached to $\sigma^{\prime}$, and whose invariant relative to $\gamma_{\beta}(\theta)$ lies in the subset $\mathcal{E}_{\beta}\left(T_{\beta}\right)$ of $\mathcal{E}\left(T_{\beta}\right)$. By Corollary $4.3, \Gamma_{\beta}\left(M_{\beta}, \theta\right)$ consists of the classes of elements of the form $\gamma_{\beta}\left(C_{\beta}, \theta\right)$, where $\gamma$ ranges over the given stable class in $\Gamma_{\text {reg }}(M)$, and $C_{\beta}$ ranges over inverse Cayley transforms. Since the right hand side of the original limit (4.7) does not depend on the choice of Cayley transform, we can sum over the set of $G_{\gamma}(\mathbb{R})$-conjugacy classes of Cayley transforms, a set whose order $d_{\alpha}=d_{\beta}$ is independent of $\gamma$, provided that we 
divide by the order $d_{\beta}$. Changing notation, we write $\gamma_{\beta}$ in place of $\gamma_{\beta}\left(C_{\beta}, \theta\right)$. The expression (4.17) then equals

$$
d_{\beta}^{-1} \lim _{\theta \rightarrow 0} \varepsilon(\theta) \sum_{\gamma_{\beta} \in \Gamma_{\beta}\left(M_{\beta}, \theta\right)} \Delta_{M_{\beta}}\left(\sigma_{\beta}^{\prime}(\theta), \gamma_{\beta}\right) D_{\beta} I_{M_{\beta}}\left(\gamma_{\beta}, f\right) .
$$

Keep in mind that the differential operator $D_{\beta}$ here is attached to $D^{\prime}$ and $\gamma_{\beta}$, according to our earlier conventions. It is defined in either of two equivalent ways by the commutative diagram (4.14).

As an endoscopic datum in $\mathcal{E}_{M^{\prime}}^{\beta}\left(M_{\beta}\right), M_{\beta}^{\prime}$ corresponds to a point $s_{\beta}^{\prime}$ in the subquotient

$$
\mathcal{K}_{\beta}^{+}=\widehat{Z}_{\beta}^{+} / Z\left(\widehat{M}_{\beta}\right)^{\Gamma}
$$

of $s_{M}^{\prime} Z(\widehat{M})^{\Gamma}$ on which the group $\mathcal{K}_{\beta}$ acts simply transitively. If $s$ belongs to $\mathcal{K}_{\beta}$, we write $M_{\beta, s}^{\prime}$ for the datum in $\mathcal{E}_{M^{\prime}}^{\beta}\left(M_{\beta}\right)$ attached to the point $s_{\beta}^{\prime} s$, and $\sigma_{\beta, s}^{\prime}(\theta)$ for a representative of the corresponding stable class in $M_{\beta, s}^{\prime}$. Suppose that $\gamma_{\beta}$ belongs to $\Gamma_{\beta}\left(M_{\beta}, \theta\right)$. By Lemma 4.2 , we have

$$
\Delta_{M_{\beta}}\left(\sigma_{\beta, s}^{\prime}(\theta), \gamma_{\beta}\right)=\Delta_{M}\left(\sigma^{\prime}, \gamma\right)=\Delta_{M_{\beta}}\left(\sigma_{\beta}^{\prime}(\theta), \gamma_{\beta}\right)
$$

for some $\gamma \in \Gamma_{\text {reg }}(M)$. We also have

$$
\kappa\left(\sigma_{\beta, s}^{\prime}(\theta), \gamma_{\beta}\right)=\kappa_{\beta}^{\prime} s,
$$

where $\kappa_{\beta}^{\prime}=\kappa\left(\sigma_{\beta}^{\prime}(\theta), \gamma_{\beta}\right)$. If $\gamma_{\beta, 1}$ is a general element in the $M_{\beta}$-stable class of $\gamma_{\beta}$, an application of (4.10) to $M_{\beta}$ tells us that

$$
\begin{aligned}
& \Delta_{M_{\beta}}\left(\sigma_{\beta, s}^{\prime}(\theta), \gamma_{\beta, 1}\right) \\
& =\Delta_{M_{\beta}}\left(\sigma_{\beta, s}^{\prime}(\theta), \gamma_{\beta}\right)\left\langle\kappa_{\beta}^{\prime} s, \operatorname{inv}\left(\gamma_{\beta}, \gamma_{\beta, 1}\right)\right\rangle^{-1} \\
& =\Delta_{M}\left(\sigma^{\prime}, \gamma\right)\left\langle\kappa_{\beta}^{\prime}, \operatorname{inv}\left(\gamma_{\beta}, \gamma_{\beta, 1}\right)\right\rangle^{-1}\left\langle s, \operatorname{inv}\left(\gamma_{\beta}, \gamma_{\beta, 1}\right)\right\rangle^{-1} .
\end{aligned}
$$

Suppose that $\gamma_{\beta, 1}$ lies in the complement of $\Gamma_{\beta}\left(M_{\beta}, \theta\right)$. As a function of $s \in \mathcal{K}_{\beta}$, this product is then a nontrivial affine character on $\mathcal{K}_{\beta}$. Its sum over $s$ vanishes. We can therefore inflate the last sum over $\gamma_{\beta}$ from $\Gamma_{\beta}\left(M_{\beta}, \theta\right)$ to $\Gamma_{G \text {-reg }}\left(M_{\beta}\right)$, provided that we also replace $\sigma_{\beta}^{\prime}(\theta)$ by $\sigma_{\beta, s}^{\prime}(\theta)$, and then take the normalized sum over $s \in \mathcal{K}_{\beta}$. For a general element $\gamma_{\beta}$ of which $\sigma_{\beta}^{\prime}(\theta)$ is an image, we can still define objects $T_{\beta}$ and $D_{\beta}$ by the natural transfer of the corresponding objects attached to any element in $\Gamma_{\beta}\left(M_{\beta}, \theta\right)$, or equivalently, by the transfer of objects $T_{\beta, s}^{\prime}$ and $D_{\beta, s}^{\prime}$ attached to $\sigma_{\beta, s}^{\prime}(\theta)$ (defined by the upper horizontal and right hand vertical arrows in (4.14)).

We have now shown that our expression for the right hand side of (4.8) can be written as the product of the constant

$$
\varepsilon_{\beta}=d_{\beta}^{-1}\left|\mathcal{K}_{\beta}\right|^{-1}
$$

with the limit

$$
\lim _{\theta \rightarrow 0} \varepsilon(\theta) \sum_{s \in \mathcal{K}_{\beta}} \sum_{\gamma_{\beta} \in \Gamma_{G-\text { reg }}\left(M_{\beta}\right)} \Delta_{M_{\beta}}\left(\sigma_{\beta, s}^{\prime}(\theta), \gamma_{\beta}\right) D_{\beta} I_{M_{\beta}}\left(\gamma_{\beta}, f\right) .
$$

Recall that $d_{\beta}$ is equal to the order of $\beta^{\vee}$ as an element in $\mathcal{E}\left(T_{\beta}\right)$. An inspection of the diagrams (4.11) and (4.12) reveals that the constant $\varepsilon_{\beta}$ then equals the order of $\mathcal{E}(T)$ divided by that of $\mathcal{E}\left(T_{\beta}\right)$. In the last limit, the outer sum over $s$ can be replaced by a sum over $M_{\beta}^{\prime}$ in $\mathcal{E}_{M^{\prime}}^{\beta}\left(M_{\beta}\right)$, if we replace $\sigma_{\beta, s}^{\prime}(\theta)$ by the associated point $\sigma_{\beta}^{\prime}(\theta)$ defined by a Cayley transform in the given group $M_{\beta}^{\prime}$. The differential 
operator $D_{\beta}$ on $C^{\infty}\left(T_{\beta}, \zeta\right)$ transfers to the operator $D_{\beta}^{\prime}$ on $C^{\infty}\left(T_{\beta}^{\prime}, M_{\beta}, \zeta\right)$ attached to $M_{\beta}^{\prime}$, which is independent of $\gamma_{\beta}$, and is to be taken outside the inner sum. In other words, we can write

$$
D_{\beta}^{\prime} I_{M_{\beta}}\left(\sigma_{\beta}^{\prime}(\theta), f\right)=\sum_{\gamma_{\beta} \in \Gamma_{G^{-\mathrm{reg}}}\left(M_{\beta}\right)} \Delta_{M_{\beta}}\left(\sigma_{\beta}^{\prime}(\theta), \gamma_{\beta}\right) D_{\beta} I_{M_{\beta}}\left(\gamma_{\beta}, f\right) .
$$

(Like (4.8), this step can be regarded as a variant of the relation (2.4), which applies to differential operators that are not symmetric under the Weyl group.) The limit becomes

$$
\lim _{\theta \rightarrow 0} \sum_{M_{\beta}^{\prime} \in \mathcal{E}_{M^{\prime}}^{\beta}\left(M_{\beta}\right)} \varepsilon(\theta) D_{\beta}^{\prime} I_{M_{\beta}}\left(\sigma_{\beta}^{\prime}(\theta), f\right) .
$$

We have at last obtained a satisfactory formula for the jump in (4.8). Let us write

$$
\varepsilon_{\beta}(\theta)=\varepsilon_{\beta} \varepsilon(\theta)=|\mathcal{E}(T)|\left|\mathcal{E}\left(T_{\beta}\right)\right|^{-1}(-i \operatorname{sgn} \theta) .
$$

Our formula is then

$$
j_{\beta^{\prime}}\left(D^{\prime} I_{M}^{\beta}\left(\sigma^{\prime}, f\right)\right)=\lim _{\theta \rightarrow 0} \varepsilon_{\beta}(\theta) \sum_{M_{\beta}^{\prime}} D_{\beta}^{\prime} I_{M_{\beta}}\left(\sigma_{\beta}^{\prime}(\theta), f\right),
$$

where $M_{\beta}^{\prime}$ is summed over the set $\mathcal{E}_{M^{\prime}}^{\beta}\left(M_{\beta}\right)$.

Proposition 4.4. Suppose that $M^{\prime}, T^{\prime}, \beta^{\prime}, \sigma^{\prime}$ and $D^{\prime}$ are as above, and that $M_{\beta}^{\prime}$ represents a variable element in $\mathcal{E}_{M^{\prime}}^{\beta}\left(M_{\beta}\right)$.

(a) If $G$ is arbitrary,

$$
j_{\beta^{\prime}}\left(D^{\prime} I_{M}^{\mathcal{E}, \beta}\left(\sigma^{\prime}, f\right)\right)=\lim _{\theta \rightarrow 0} \varepsilon_{\beta}(\theta) \sum_{M_{\beta}^{\prime}} D_{\beta}^{\prime} I_{M_{\beta}}^{\mathcal{E}}\left(\sigma_{\beta}^{\prime}(\theta), f\right) .
$$

(b) If $G$ is quasisplit,

$$
j_{\beta^{\prime}}\left(D^{\prime} S_{M}^{G, \beta}\left(M^{\prime}, \sigma^{\prime}, f\right)\right)=\lim _{\theta \rightarrow 0} \varepsilon_{\beta}(\theta) \sum_{M_{\beta}^{\prime}} D_{\beta}^{\prime} S_{M_{\beta}}^{G}\left(M_{\beta}^{\prime}, \sigma_{\beta}^{\prime}(\theta), f\right) .
$$

Remarks. 1. It is implicit in the assertions that the half limits defined by the left hand sides of the two formulas all exist.

2. In the special case that $M^{\prime}=M^{*}$, Theorem $1.1(\mathrm{~b})$ asserts that the sum in (4.21) can be taken over the single element $M_{\beta}^{\prime}=M_{\beta}^{*}$. Assuming the assertion, the formula (4.20) could then be written in this case as

$$
j_{\beta}\left(D S_{M}^{G, \beta}(\sigma, f)\right)=\lim _{\theta \rightarrow 0} \varepsilon_{\beta}(\theta) D_{\beta} S_{M_{\beta}}^{G}\left(\sigma_{\beta}(\theta), f\right),
$$

where $D, \sigma$ and $D_{\beta}$ denote analogues for $G$ of the objects $D^{\prime}=D^{*}, \sigma^{\prime}=\sigma^{*}$ and $D_{\beta}^{\prime}=D_{\beta}^{*}$.

Proof. We assume inductively that the analogue of (b) holds for any pair $\left(G^{\prime}, M^{\prime}\right)$ in which $G^{\prime}$ lies in both $\mathcal{E}_{M^{\prime}}^{0}(G)$ and $\mathcal{E}_{M^{\prime}}^{\beta}(G)$. At the end of $\S 2$, we took on a similar induction hypothesis for the assertion of Theorem 1.1(b). This means that the analogue

$$
j_{\beta^{\prime}}\left(D^{\prime} \widehat{S}_{\tilde{M}^{\prime}}^{\tilde{G}^{\prime}, \beta^{\prime}}\left(\sigma^{\prime}, f^{\prime}\right)\right)=\lim _{\theta \rightarrow 0} \varepsilon_{\beta^{\prime}}(\theta) D_{\beta}^{\prime} \widehat{S}_{\tilde{M}_{\beta}^{\prime}}^{\tilde{G}^{\prime}}\left(\sigma_{\beta}^{\prime}(\theta), f^{\prime}\right)
$$


of (4.22) for $\left(G^{\prime}, M^{\prime}\right)$ holds. If $G^{\prime}$ belongs to the complement of $\mathcal{E}_{M^{\prime}}^{\beta}(G), \beta^{\prime}$ is not a root of $\left(G^{\prime}, T^{\prime}\right)$, and the point $\sigma^{\prime}$ is strongly $G^{\prime}$-regular. In this case $\widehat{S}_{\tilde{M}}^{\tilde{G}^{\prime}}, \beta^{\prime}\left(\sigma^{\prime}, f^{\prime}\right)$ is smooth at $\sigma^{\prime}$, and the jump on the left hand side of (4.23) vanishes.

The proof is now similar to that of Lemma 4.1. It follows from Lemma 4.1 and the definition of $j_{\beta^{\prime}}$ that the difference

$$
j_{\beta^{\prime}}\left(D^{\prime} I_{M}^{\mathcal{E}, \beta}\left(\sigma^{\prime}, f\right)\right)-\varepsilon(G) j_{\beta^{\prime}}\left(D^{\prime} S_{M}^{G, \beta}\left(M^{\prime}, \sigma^{\prime}, f\right)\right)
$$

equals

$$
\sum_{G^{\prime} \in \mathcal{E}_{M^{\prime}}^{0}(G)} \iota_{M^{\prime}}\left(G, G^{\prime}\right) j_{\beta^{\prime}}\left(D^{\prime} \widehat{S}_{\tilde{\tilde{M}^{\prime}}}^{\tilde{G}^{\prime}, \beta^{\prime}}\left(\sigma^{\prime}, f^{\prime}\right)\right)
$$

The last expression can in turn be written as

$$
\sum_{G^{\prime} \in \mathcal{E}_{M^{\prime}}^{0}(G) \cap \mathcal{E}_{M^{\prime}}^{\beta}(G)} \iota_{M^{\prime}}\left(G, G^{\prime}\right)\left(\lim _{\theta \rightarrow 0} \varepsilon_{\beta^{\prime}}(\theta) D_{\beta}^{\prime} \widehat{S}_{\tilde{M}_{\beta}^{\prime}}^{\tilde{G}^{\prime}}\left(\sigma_{\beta}^{\prime}(\theta), f^{\prime}\right)\right),
$$

by the discussion above. Following the proof of Lemma 4.1, we decompose the last sum over $G^{\prime}$ into a double sum over $M_{\beta}^{\prime} \in \mathcal{E}_{M^{\prime}}^{\beta}\left(M_{\beta}\right)$ and $G_{\beta}^{\prime} \in \mathcal{E}_{M_{\beta}^{\prime}}^{0}(G)$, and write

$$
\iota_{M^{\prime}}\left(G, G^{\prime}\right)=\iota_{M^{\prime}}\left(M_{\beta}, M_{\beta}^{\prime}\right) \iota_{M_{\beta}^{\prime}}\left(G, G_{\beta}^{\prime}\right) .
$$

We then write

$$
\begin{aligned}
\iota_{M^{\prime}} & \left(M_{\beta}, M_{\beta}^{\prime}\right) \varepsilon_{\beta^{\prime}}(\theta) \\
= & \left|Z\left(\widehat{M}^{\prime}\right)^{\Gamma} / Z(\widehat{M})^{\Gamma}\right| \cdot\left|Z\left(\widehat{M}_{\beta}^{\prime}\right)^{\Gamma_{\beta}} / Z\left(\widehat{M}_{\beta}\right)^{\Gamma_{\beta}}\right|^{-1} \\
& \cdot\left|\left(\widehat{T}^{\prime}\right)^{\Gamma} / Z\left(\widehat{M}^{\prime}\right)^{\Gamma}\right| \cdot\left|\left(\widehat{T}_{\beta}^{\prime}\right)^{\Gamma_{\beta}} / Z\left(\widehat{M}_{\beta}^{\prime}\right)^{\Gamma_{\beta}}\right|^{-1} \varepsilon(\theta) \\
= & \left|\left(T^{\prime}\right)^{\Gamma} / Z(\widehat{M})^{\Gamma}\right| \cdot\left|\left(\widehat{T}_{\beta}^{\prime}\right)^{\Gamma_{\beta}} / Z\left(\widehat{M}_{\beta}\right)^{\Gamma_{\beta}}\right|^{-1} \\
= & \left|\widehat{T}^{\Gamma} / Z(\widehat{M})^{\Gamma}\right| \cdot\left|\widehat{T}_{\beta}^{\Gamma_{\beta}} / Z\left(\widehat{M}_{\beta}\right)^{\Gamma_{\beta}}\right|^{-1} \varepsilon(\theta) \\
= & \varepsilon_{\beta}(\theta) .
\end{aligned}
$$

The difference (4.24) therefore equals the sum over $M_{\beta}^{\prime}$ of the expression

$$
\sum_{G_{\beta}^{\prime}} \lim _{\theta \rightarrow 0} \varepsilon_{\beta}(\theta) \iota_{M_{\beta}}\left(G^{\prime}, G_{\beta}^{\prime}\right) D_{\beta}^{\prime} \widehat{S}_{\tilde{M}_{\beta}^{\prime}}^{\tilde{G}^{\prime}}\left(\sigma_{\beta}^{\prime}(\theta), f^{\prime}\right), \quad G_{\beta}^{\prime} \in \mathcal{E}_{M_{\beta}^{\prime}}^{0}(G) .
$$

The last step is to take the limit operation outside the two sums, and then apply the definitions (1.8)-(1.10) (with $M_{\beta}$ in place of $M$ ) to the resulting sum over $G_{\beta}$. We conclude that the difference (4.24) equals the limit

$$
\lim _{\theta \rightarrow 0} \varepsilon_{\beta}(\theta) \sum_{M_{\beta}^{\prime}}\left(D_{\beta}^{\prime} I_{M_{\beta}}^{\mathcal{E}}\left(\sigma_{\beta}^{\prime}(\theta), f\right)-\varepsilon(G) D_{\beta}^{\prime} S_{M}^{G}\left(M_{\beta}^{\prime}, \sigma_{\beta}^{\prime}(\theta), f\right)\right) .
$$

If $G$ is not quasisplit, $\varepsilon(G)=0$. The equality of (4.24) and (4.25) then reduces to the required formula (4.20) of (a). If $G$ is quasisplit, $\varepsilon(G)=1$, and the formula of (a) follows from (4.19) and the definition (1.10). In this case, the equality of (4.24) and (4.25) reduces to the required formula (4.21) of (b). 


\section{§5. Stabilization OF The ASYMPtotic FORMula}

There is one more ingredient we need for our proof of Theorem 1.1. It is the invariant asymptotic formula

$$
\lim _{T \underset{P, r}{\longrightarrow} \infty} I_{M}\left(\gamma_{T}, f_{T}\right)=\int_{T_{\varepsilon_{P}}(M, \zeta)} \theta_{M}(\gamma, \tau) I_{M}^{P}(\tau, f) d \tau
$$

that was the main result [A14, Corollary 6.2] of the last paper. We can in fact regard this formula as a boundary condition at infinity in the noncompact torus $T(\mathbb{R})$. An essential object to be stabilized here is the linear form

$$
I_{M}^{P}(\gamma, f)=\int_{T_{\varepsilon_{P}}(M, \zeta)} \theta_{M}(\gamma, \tau) I_{M}^{P}(\tau, f) d \tau
$$

that occurs on the right hand side of the formula. We begin by recalling some of the terms in the formula, as we will be applying them here in slightly greater generality.

First of all, the function $f$ in (5.1) and (5.2) has to be taken from a subspace of $\mathcal{C}(G, \zeta)$ for the formulas to make sense. It suffices to let $f$ be a function in the $\zeta^{-1}$-equivariant Hecke algebra

$$
\mathcal{H}(G, \zeta)=\bigoplus_{\iota \in \pi_{0}(G)} \mathcal{H}\left(G_{\iota}, \zeta_{\iota}\right)
$$

on $G(\mathbb{R})$. The mapping $f \rightarrow f_{G}$ takes $\mathcal{H}(G, \zeta)$ to a subspace

$$
I \mathcal{H}(G, \zeta)=\bigoplus_{\iota} I \mathcal{H}\left(G_{\iota}, \zeta_{\iota}\right)
$$

of $\mathcal{I}(G, \zeta)$. As a space of functions on $\Pi_{\text {temp }}(G, \zeta), I \mathcal{H}(G, \zeta)$ was characterized in CD. One can also identify $I \mathcal{H}(G, \zeta)$ with the Paley-Wiener space on the space of virtual characters

$$
T_{\text {temp }}(G, \zeta)=\coprod_{\iota} T_{\text {temp }}\left(G_{\iota}, \zeta_{\iota}\right)
$$

(See A6]. For any $\iota, T_{\text {temp }}\left(G_{\iota}, \zeta_{\iota}\right)$ is the subset of virtual characters in the set denoted $T\left(G_{\iota}(\mathbb{R})\right)$ in $\left[\mathrm{A} 6\right.$ ] whose $Z_{\iota}(\mathbb{R})$-central character equals $\zeta_{\iota}$.)

We write $\mathfrak{a}_{M, Z}^{*}$ for the kernel of the projection of $\mathfrak{a}_{M}^{*}$ onto $\mathfrak{a}_{Z}^{*}$. There are then free actions $\pi \rightarrow \pi_{\lambda}$ and $\tau \rightarrow \tau_{\lambda}$ of $i \mathfrak{a}_{M, Z}^{*}$ on the sets $\Pi_{\text {temp }}(M, \zeta)$ and $T_{\text {temp }}(M, \zeta)$. These mappings can obviously also be defined if $\lambda$ is any element in the complexification $a_{M, Z, \mathbb{C}}^{*}$ of $\mathfrak{a}_{M, Z}^{*}$, but their images will then consist of nontempered virtual characters. If $\varepsilon$ belongs to the real space $\mathfrak{a}_{M, Z}^{*}$, we write $T_{\varepsilon}(M, \zeta)$ for the set of virtual characters

$$
\left\{\tau_{\lambda}: \lambda \in \varepsilon+i \mathfrak{a}_{M, Z}^{*}, \tau \in T_{\text {temp }}(M, \zeta)\right\} .
$$

The asymptotic formula (5.1) depends on a fixed parabolic subgroup $P \in \mathcal{P}(M)$. The domain of integration on the right hand side of the formula is then defined by a small point $\varepsilon=\varepsilon_{P}$ in general position in the corresponding chamber $\left(\mathfrak{a}_{M, Z}^{*}\right)_{P}^{+}$in $\mathfrak{a}_{M, Z}^{*}$. The limit on the left hand side is over points $T$ in the set

$$
\mathfrak{a}_{P}^{r}=\left\{H \in \mathfrak{a}_{M}: \alpha(H)>r\|H\|, \alpha \in \Delta_{P}\right\},
$$

defined by the simple roots $\Delta_{P}$ of $\left(P, A_{M}\right)$, and a fixed, small positive number $r$. 
The other ingredients in (5.1) are given essentially by the definitions of A14. For example

$$
\gamma_{T}=\gamma \exp T
$$

while $f \rightarrow f_{T}$ is the isomorphism of $\mathcal{C}(G, \zeta)$ defined as in $\S 1$ of A14. The function $\theta_{M}(\gamma, \tau)$ is the kernel of the transformation

$$
a_{M}(\gamma)=\int_{T_{\text {temp }}(M, \zeta)} \theta_{M}(\gamma, \tau) a_{M}(\tau) d \tau, \quad a_{M} \in \mathcal{I}(M, \zeta),
$$

that relates to two ways of viewing a function in $\mathcal{I}(M, \zeta)$. The linear form $I_{M}^{P}(\tau, f)$ is the invariant distribution

$$
I_{M}^{P}(\tau, f)=\operatorname{tr}\left(m_{M}(\tau, P) \mathcal{I}_{P}(\tau, f)\right)=m_{M}(\tau, P) f_{M}(\tau),
$$

where

$$
f_{M}(\tau)=f_{G}\left(\tau^{G}\right)
$$

is the restriction of $f_{G}$ to the induced image of $T_{\text {temp }}(M, \zeta)$ in $T_{\text {temp }}(G, \zeta)$, and $m_{M}(\tau, P)$ is defined in terms of Plancherel densities as in [A14, §6].

What does it mean to stabilize $I_{M}^{P}(\gamma, f)$ ? In theory, the process entails repeating the definitions of $\S 1$ with $I_{M}^{P}(\gamma, f)$ in place of $I_{M}(\gamma, f)$. However, the distributions $I_{M}^{P}(\gamma, f)$ are much simpler than the original ones. The basic step will be to stabilize the function $m_{M}(\tau, P)$ by an analogue of Theorem 5 of A10.

We write $\Phi_{\text {temp }}(M, \zeta)$ for the set of ( $\widehat{M}$-orbits of $)$ tempered Langlands parameters

$$
\phi: W_{\mathbb{R}} \longrightarrow{ }^{L} M
$$

whose central character on $Z(\mathbb{R})$ equals $\zeta$. Any such parameter $\phi$ determines a finite packet

$$
\Pi_{\phi}=\coprod_{\iota} \Pi_{\phi_{\iota}}, \quad \Pi_{\phi_{\iota}} \subset \Pi_{\mathrm{temp}}\left(M_{\iota}, \zeta_{\iota}\right),
$$

of representations in $\Pi_{\text {temp }}(M, \zeta)$, and a finite packet

$$
T_{\phi}=\coprod_{\iota} T_{\phi_{\iota}}, \quad T_{\phi_{\iota}} \subset T_{\mathrm{temp}}\left(M_{\iota}, \zeta_{\iota}\right)
$$

of virtual representations in $T_{\text {temp }}(M, \zeta)$. The packet $\Pi_{\phi_{\iota}}$ for the connected group $M_{\iota}$ is defined as in [L3], with the understanding that it is empty if $\phi_{\iota}$ is not relevant to $M_{\iota}$. The packet $T_{\phi_{\iota}}$ is defined as the subset of $T_{\text {temp }}(M, \zeta)$ whose linear span equals that of $\Pi_{\phi_{l}}$. The Langlands classification for real groups asserts that both $\Pi_{\text {temp }}(M, \zeta)$ and $T_{\text {temp }}(M, \zeta)$ can be decomposed into disjoint unions over $\phi$ of the associated packets. Our notation is not completely standard here, since $\Phi_{\text {temp }}(M, \zeta)$ usually denotes the subset of parameters $\phi$ that are relevant to $M$. In the present context, this means that any Levi subgroup of ${ }^{L} M$ that contains the image of $\phi$ is dual to a Levi $K$-subgroup of $M$, or equivalently, that the packet $\Pi_{\phi}$ (or $T_{\phi}$ ) is nonempty.

There is a free action $\phi \rightarrow \phi_{\lambda}$ of $i \mathfrak{a}_{M, Z}^{*}$ on $\Phi_{\text {temp }}(M, \zeta)$, obtained by identifying $\mathfrak{a}_{M, Z, \mathbb{C}}^{*}$ with a subspace of the Lie algebra of $Z(\widehat{M})^{\Gamma}$. This action is compatible with the two kinds of packets, and the two actions of $i \mathfrak{a}_{M, Z}^{*}$ on $\Pi_{\text {temp }}(M, \zeta)$ and $T_{\text {temp }}(M, \zeta)$. It again extends to the complexification $\mathfrak{a}_{M, Z, \mathbb{C}}^{*}$, but if $\lambda$ lies in the complement of $i \mathfrak{a}_{M, Z}^{*}, \phi \rightarrow \phi_{\lambda}$ maps $\Phi_{\text {temp }}(M, \zeta)$ to its complement in the set 
$\Phi(M, \zeta)$ of general Langlands parameters. We again write

$$
\Phi_{\varepsilon}(M, \zeta)=\left\{\phi_{\lambda}: \lambda \in \varepsilon+i \mathfrak{a}_{M, Z}^{*}, \phi \in \Phi_{\text {temp }}(M, \zeta)\right\}
$$

for any point $\varepsilon$ in $\mathfrak{a}_{M, Z}^{*}$.

Suppose that $\left\{\tau_{\lambda}\right\}$ is the $\mathfrak{a}_{M, Z, \mathbb{C}^{-}}^{*}$ orbit of an element $\tau=\tau_{0}$ in $T_{\text {temp }}(M, \zeta)$. The set of values $\left\{m_{M}\left(\tau_{\lambda}, P\right)\right\}$ assumed by the function used to define $I_{M}^{P}(\tau, f)$ represents a meromorphic function of $\lambda$, whose restriction to $i \mathfrak{a}_{M, Z}^{*}$ is analytic. It is defined in terms of the (inverses of) Plancherel densities

$$
m_{Q \mid P}\left(\tau_{\lambda}\right)=\mu_{Q \mid P}\left(\tau_{\lambda}\right)^{-1}, \quad Q \in \mathcal{P}(M),
$$

attached to the virtual character $\tau_{\lambda}$. One forms the $(G, M)$-family

$$
m_{Q}\left(\Lambda, \tau_{\lambda}, P\right)=m_{Q \mid P}\left(\tau_{\lambda}\right)^{-1} m_{Q \mid P}\left(\tau_{\lambda+\frac{1}{2} \Lambda}\right), \quad Q \in \mathcal{P}(M), \Lambda \in i \mathfrak{a}_{M, Z}^{*},
$$

and then defines the function $m_{M}\left(\tau_{\lambda}, P\right)$ as the associated limit

$$
m_{M}\left(\tau_{\lambda}, P\right)=\lim _{\Lambda \rightarrow 0} \sum_{Q \in \mathcal{P}(M)} m_{Q}\left(\Lambda, \tau_{\lambda}, P\right) \theta_{Q}(\Lambda)^{-1} .
$$

(See [A14, $\S 5,6]$. The function $\theta_{Q}(\Lambda)$ is a homogeneous polynomial in $\Lambda$ unrelated to the kernel $\theta_{M}(\gamma, \tau)$.) Let $\phi \in \Phi_{\text {temp }}(M, \zeta)$ be the parameter such that $\tau$ lies in $T_{\phi}$. The functions $m_{Q \mid P}\left(\phi_{\lambda}\right)=m_{Q \mid P}\left(\tau_{\lambda}\right)$ then depend on $\tau_{\lambda}$ through $\phi_{\lambda}$. To be more precise, let $\rho_{Q \mid P}$ be the representation of ${ }^{L} M$ on the intersection of the Lie algebras of the unipotent radicals of $\widehat{\bar{P}}$ and $\widehat{Q}$. If $\lambda$ is purely imaginary, the inverse Plancherel density is defined explicitly in terms of archimedean $L$-functions by

$$
m_{Q \mid P}\left(\phi_{\lambda}\right)=c_{Q \mid P}\left|L\left(0, \rho_{Q \mid P} \circ \phi_{\lambda}\right)\right|^{2}\left|L\left(1, \rho_{Q \mid P} \circ \phi_{\lambda}\right)\right|^{-2},
$$

where $c_{Q \mid P}$ is a constant that depends only on the choice of Haar measure on $N_{\bar{P}}(\mathbb{R}) \cap N_{Q}(\mathbb{R})$ implicit in the Plancherel density [A5, §3]. For general $\lambda, m_{Q \mid P}\left(\phi_{\lambda}\right)$ is defined by meromorphic continuation of the real analytic function given by imaginary $\lambda$. Since this function depends only on $\phi$, so does the limit

$$
m_{M}\left(\phi_{\lambda}, P\right)=m_{M}\left(\tau_{\lambda}, P\right) \text {. }
$$

Suppose that $M^{\prime}$ is the elliptic endoscopic datum for $M$ fixed earlier, with auxiliary datum $\left(\widetilde{M}^{\prime}, \widetilde{\xi}_{M}^{\prime}\right)$. The embedding of $Z(\widehat{M})^{\Gamma}$ into $Z(\widehat{\widetilde{M}})^{\Gamma}$ allows us to identify $\mathfrak{a}_{M, Z, \mathbb{C}}^{*}$ with the subspace $\mathfrak{a}_{\tilde{M}^{\prime}, \tilde{Z^{\prime}}, \mathbb{C}}^{*}$ of the Lie algebra of $Z(\widehat{\widehat{M}})^{\Gamma}$. We therefore have an action $\phi^{\prime} \rightarrow \phi_{\lambda}^{\prime}$ of $i \mathfrak{a}_{M, Z}^{*}$ on $\Phi_{\text {temp }}\left(\widetilde{M}^{\prime}, \widetilde{\zeta}^{\prime}\right)$. From our point of view, the most important aspect of the Langlands parameters for $\widetilde{M}^{\prime}$ is that they form the domain of a canonical mapping $\phi^{\prime} \rightarrow \phi$ from $\Phi_{\text {temp }}\left(\widetilde{M}^{\prime}, \widetilde{\zeta}^{\prime}\right)$ to $\Phi_{\text {temp }}(M, \zeta)$. Indeed, the central character $\eta^{\prime}$ of any $\phi^{\prime}$ on $\widetilde{C}^{\prime}(\mathbb{R})$ is derived from $\widetilde{\xi}_{M}^{\prime}$ in such a way that $\phi^{\prime}$ factors to an $L$-homomorphism $\bar{\phi}^{\prime}$ from the Weil group $W_{\mathbb{R}}$ to $\mathcal{M}^{\prime}$. We define $\phi$ to be the composition $\xi_{M}^{\prime} \circ \bar{\phi}^{\prime}$ of the two horizontal arrows in the diagram

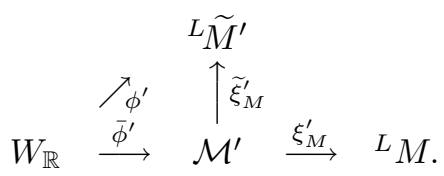

The mapping $\phi^{\prime} \rightarrow \phi$ is compatible with the actions of $i \mathfrak{a}_{M, Z}^{*}$ on $\Phi_{\text {temp }}\left(\widetilde{M}^{\prime}, \widetilde{\zeta}^{\prime}\right)$ and $\Phi(M, \zeta)$. 
The stabilization we seek takes the form of an inductive family of identities among the functions

$$
m_{M}\left(\phi_{\lambda}, P\right)=m_{M}^{G}\left(\phi_{\lambda}, P\right) .
$$

The identities are parallel to those of Proposition 2.1, and relate quantities defined inductively by varying $G, M$ and $\zeta$. In particular, they are formulated in terms of the set $\mathcal{E}_{M^{\prime}}(G)$. Recall that any element $G^{\prime} \in \mathcal{E}_{M^{\prime}}(G)$ comes with an implicit choice of embedding $M^{\prime} \subset G^{\prime}$ for which $\widehat{M}^{\prime} \subset \widehat{G}^{\prime}$ is a dual Levi subgroup. A parabolic subgroup $P^{\prime} \in \mathcal{P}^{G^{\prime}}\left(M^{\prime}\right)$ can therefore be identified with a chamber $\mathfrak{a}_{P^{\prime}}^{+}$in the space $\mathfrak{a}_{M^{\prime}}=\mathfrak{a}_{M}$. We use this to define a mapping $P \rightarrow P^{\prime}$ from $\mathcal{P}(M)$ to $\mathcal{P}^{G^{\prime}}\left(M^{\prime}\right)$ by requiring that $\mathfrak{a}_{P}^{+}$be contained in $\mathfrak{a}_{P^{\prime}}^{+}$.

As has been the case before, we shall take $\left(\widetilde{M}^{\prime}, \widetilde{\xi}_{M}^{\prime}\right)$ to be the restriction of an auxiliary datum $\left(\widetilde{G}^{\prime}, \widetilde{\xi}^{\prime}\right)$ for a given $G^{\prime} \in \mathcal{E}_{M^{\prime}}(G)$. Then $\widetilde{M}^{\prime}$ becomes a Levi subgroup of $\widetilde{G}^{\prime}$ for which the set $\mathcal{P}^{\tilde{G}^{\prime}}\left(\widetilde{M}^{\prime}\right)$ is in bijection with $\mathcal{P}^{G^{\prime}}\left(M^{\prime}\right)$. We shall let $P^{\prime}$ stand for the group in $\mathcal{P}^{\tilde{G}^{\prime}}\left(\tilde{M}^{\prime}\right)$, as well as the corresponding group in $\mathcal{P}^{G}(M)$.

Proposition 5.1. There is an identity

$$
m_{M}^{G}\left(\phi_{\lambda}, P\right)=\sum_{G^{\prime} \in \mathcal{E}_{M^{\prime}}(G)} \iota_{M^{\prime}}\left(G, G^{\prime}\right) n_{\tilde{M}^{\prime}}^{\tilde{G}^{\prime}}\left(\phi_{\lambda}^{\prime}, P^{\prime}\right),
$$

where

$$
n_{\tilde{M}^{\prime}}^{\tilde{G}^{\prime}}\left(\phi_{\lambda}^{\prime}, P^{\prime}\right), \quad G^{\prime} \in \mathcal{E}_{M^{\prime}}(G), \phi^{\prime} \in \Phi_{\text {temp }}\left(\tilde{M}^{\prime}, \widetilde{\zeta}^{\prime}\right)
$$

is a meromorphic function of $\lambda \in \mathfrak{a}_{M, Z, \mathbb{C}}^{*}$ that depends only on the quasisplit pair $\left(\widetilde{G}^{\prime}, \widetilde{M}^{\prime}\right)$, and the elements $\phi^{\prime} \in \Phi_{\text {temp }}\left(\tilde{M}^{\prime}, \widetilde{\zeta}^{\prime}\right)$ and $P^{\prime} \in \mathcal{P}^{\tilde{G}^{\prime}}\left(\tilde{M}^{\prime}\right)$.

Proof. The proposition is reminiscent of Theorem 5 of A10. Since the proof is quite similar, we can be brief.

If $G$ is quasisplit, the "stable" function

$$
n_{M}^{G}\left(\phi_{\lambda}, P\right)=n_{M^{*}}^{G^{*}}\left(\phi_{\lambda}^{*}, P^{*}\right), \quad \phi \in \Phi_{\text {temp }}(M, \zeta),
$$

is uniquely determined by the required identity. We define it inductively by setting

$$
n_{M}^{G}\left(\phi_{\lambda}, P\right)=m_{M}^{G}\left(\phi_{\lambda}, P\right)-\sum_{G^{\prime} \in \mathcal{E}_{M^{\prime}}^{0}(G)} \iota_{M^{\prime}}\left(G, G^{\prime}\right) n_{\tilde{M}^{\prime}}^{\tilde{G}^{\prime}}\left(\phi_{\lambda}^{\prime}, P^{\prime}\right),
$$

in the case $M^{\prime}=M^{*}$. Having made this definition, we then fix general objects $G$, $M, \zeta, P, M^{\prime}$ and $\phi^{\prime}$. We have to show that if $\phi$ is the image of $\phi^{\prime}$ in $\Phi_{\text {temp }}(M, \zeta)$, the original function $m_{M}^{G}\left(\phi_{\lambda}, P\right)$ equals the endoscopic expression

$$
m_{M}^{G, \mathcal{E}}\left(\phi_{\lambda}^{\prime}, P\right)=\sum_{G^{\prime} \in \mathcal{E}_{M^{\prime}}(G)} \iota_{M^{\prime}}\left(G, G^{\prime}\right) n_{\tilde{M}^{\prime}}^{\tilde{G}^{\prime}}\left(\phi_{\lambda}^{\prime}, P^{\prime}\right) .
$$

The notation in (5.5) leaves a little to be desired. The argument $\phi_{\lambda}^{\prime}$ on the left hand side should really be regarded as a family of parameters attached to $\phi_{\lambda}$, which vary with the auxiliary data $\left(\widetilde{M}^{\prime}, \widetilde{\xi}_{M}^{\prime}\right)$ and $\left(\widetilde{G}^{\prime}, \widetilde{\xi}^{\prime}\right)$ implicit in the terms on the right hand side. It is easy to check that each of these terms is in fact independent of the choice of $\left(\widetilde{G}^{\prime}, \widetilde{\xi}^{\prime}\right)$. The property follows inductively from the fact that the original function $m_{M}^{G}\left(\phi_{\lambda}, P\right)$ remains unchanged if $\phi$ is twisted by a 1-cocycle in $H^{1}\left(W_{F}, Z(\widehat{G})\right)$. As for the proof of (5.5), it suffices by analytic continuation to treat the case that $\lambda$ is purely imaginary. Following A10, we will consider a slightly more general identity. 
For any $Q \in \mathcal{P}(M)$, let

$$
\rho_{Q \mid P}=\bigoplus_{a} \rho_{a}
$$

be the decomposition of the representation $\rho_{Q \mid P}$ of $W_{\mathbb{R}}$ relative to the adjoint action of $Z(\widehat{M})^{\Gamma}$. The elements $a$ range over characters on $Z(\widehat{M})^{\Gamma}$ that are trivial on $Z(\widehat{G})^{\Gamma}$, with the subrepresentations $\rho_{a}$ being nontrivial only if $a$ lies in the intersection $\Sigma(\widehat{Q}) \cap \Sigma(\widehat{\bar{P}})$ of the sets of roots of $\widehat{Q}$ and $\widehat{\bar{P}}$. Then

$$
L\left(s, \rho_{Q \mid P} \circ \phi_{\lambda}\right)=\prod_{a} L\left(s, \rho_{a} \circ \phi_{\lambda}\right) .
$$

Since $\lambda$ is purely imaginary, we can then write

$$
m_{Q \mid P}\left(\phi_{\lambda}\right)=\prod_{a} m_{a}\left(\phi_{\lambda}\right)
$$

for functions

$$
m_{a}\left(\phi_{\lambda}\right)=m_{a}^{G}\left(\phi_{\lambda}\right)=c_{a}\left|L\left(0, \rho_{a} \circ \phi_{\lambda}\right)\right|^{2}\left|L\left(1, \rho_{a} \circ \phi_{\lambda}\right)\right|^{-2},
$$

defined for constants $c_{a}$ whose product equals $c_{Q \mid P}$. The constants are actually irrelevant, since it is only a logarithmic derivative of $m_{a}\left(\phi_{\lambda}\right)$ that contributes to the function $m_{M}^{G}\left(\phi_{\lambda}, P\right)$.

Suppose that $a$ represents any character on $Z(\widehat{M})^{\Gamma} / Z(\widehat{G})^{\Gamma}$. The kernel $\widehat{Z}_{a}$ of $a$ in $Z(\widehat{M})^{\Gamma}$ acts by translation on $Z(\widehat{M})^{\Gamma} / Z(\widehat{G})^{\Gamma}$, and hence on $\mathcal{E}_{M^{\prime}}(G)$. We write $\mathcal{E}_{M^{\prime}}(G) / \widehat{Z}_{a}$ for the set of orbits. If $G^{\prime} \in \mathcal{E}_{M^{\prime}}(G)$ is elliptic, the canonical mapping from $Z(\widehat{M})^{\Gamma} / Z(\widehat{G})^{\Gamma}$ to $Z\left(\widehat{\widetilde{M}}^{\prime}\right)^{\Gamma} / Z\left(\widehat{\widetilde{G}}^{\prime}\right)^{\Gamma}$ is surjective with finite kernel, and if $a$ is trivial on the kernel, it transfers to a unique character $a^{\prime}$ on $Z\left(\widehat{\widetilde{M}}^{\prime}\right)^{\Gamma} / Z\left(\widetilde{\widetilde{G}}^{\prime}\right)^{\Gamma}$. One establishes a decomposition

$$
L\left(s, \rho_{a} \circ \phi_{\lambda}\right)=\prod_{G^{\prime} \in \mathcal{E}_{M^{\prime}}(G) / \widehat{Z}_{a}} L\left(s, \rho_{a^{\prime}} \circ \phi_{\lambda}^{\prime}\right),
$$

in which the factor corresponding to $G^{\prime}$ is understood to be 1 unless $G^{\prime}$ is elliptic and $a$ transfers to a character $a^{\prime}$ in this way. The decomposition follows from the proof of Lemma 4 of A10 with the family of conjugacy classes $c^{\prime}$ in A10 replaced by the Langlands parameter $\phi_{\lambda}^{\prime}$ here. (One observes that the factors do depend only on the $\widehat{Z}_{a^{\text {-orbits }}}$ in $\mathcal{E}_{M^{\prime}}(G)$ and in the special case of $M^{\prime}=M^{*}$, that there is only one nontrivial factor.) We can then arrange that

$$
m_{a}^{G}\left(\phi_{\lambda}\right)=\prod_{G^{\prime} \in \mathcal{E}_{M^{\prime}}(G) / \widehat{Z}_{a}} m_{a^{\prime}}^{\tilde{G}^{\prime}}\left(\phi_{\lambda}^{\prime}\right)
$$

by choosing the constants $\left\{c_{a^{\prime}}=c_{a^{\prime}}^{\tilde{G}^{\prime}}\right\}$ appropriately.

The generalization of (5.5) is provided by a finite set $A$ of characters on $Z(\widehat{M})^{\Gamma} / Z(\widehat{G})^{\Gamma}$. As in p. 1144 of [A10], we define a $(G, M)$-family

$$
m_{Q}\left(\Lambda, \phi_{\lambda}, P, A\right)=\prod_{a \in A \cap \Sigma(\widehat{Q}) \cap \Sigma(\hat{\bar{P}})} m_{a}\left(\phi_{\lambda}\right)^{-1} m_{a}\left(\phi_{\lambda+\frac{1}{2} \Lambda}\right)
$$

of functions of $\Lambda \in i \mathfrak{a}_{M, Z}^{*}$, with values in the space of meromorphic functions of $\lambda$. This yields in turn a meromorphic function $m_{M}^{G}\left(\phi_{\lambda}, P, A\right)$ by the analogue of 
the limit (5.3). We define generalizations $n_{M}^{G}\left(\phi_{\lambda}, P, A\right)$ and $m_{M}^{G, \mathcal{E}}\left(\phi_{\lambda}^{\prime}, P, A\right)$ of the original functions

and

$$
n_{M}^{G}\left(\phi_{\lambda}, P\right)=n_{M}^{G}\left(\phi_{\lambda}, P, \Sigma(\widehat{\bar{P}})\right)
$$

$$
m_{M}^{G, \mathcal{E}}\left(\phi_{\lambda}^{\prime}, P\right)=m_{M}^{G, \mathcal{E}}\left(\phi_{\lambda}^{\prime}, P, \Sigma(\widehat{\bar{P}})\right)
$$

by setting

$$
n_{M}^{G}\left(\phi_{\lambda}, P, A\right)=m_{M}^{G}\left(\phi_{\lambda}, P, A\right)-\sum_{G^{\prime} \in \mathcal{E}_{M^{*}}^{0}(G)} \iota_{M^{*}}\left(G, G^{\prime}\right) n_{\tilde{M}^{\prime}}^{\tilde{G}^{\prime}}\left(\phi_{\lambda}^{*}, P^{*}, A^{*}\right)
$$

for $G$ quasisplit, and

$$
m_{M}^{G, \mathcal{E}}\left(\phi_{\lambda}^{\prime}, P, A\right)=\sum_{G^{\prime} \in \mathcal{E}_{M^{\prime}}(G)} \iota_{M^{\prime}}\left(G, G^{\prime}\right) n_{\tilde{M}^{\prime}}^{\tilde{G}^{\prime}}\left(\phi_{\lambda}^{\prime}, P^{\prime}, A^{\prime}\right)
$$

in general. We have written $A^{\prime}$ here for the set of characters $a^{\prime}$ on $Z\left(\widehat{\widetilde{M}}^{\prime}\right)^{\Gamma} / Z\left(\widehat{\widetilde{G}}^{\prime}\right)^{\Gamma}$ obtained as above from elements $a \in A$. These definitions set the stage for proving the equality of $m_{M}^{G, \mathcal{E}}\left(\phi_{\lambda}^{\prime}, P, A\right)$ and $m_{M}^{G}\left(\phi_{\lambda}, P, A\right)$ by induction on the number of elements in $A$.

The main step is when $A$ consists of one element $a$. In this case, the relevant functions $m_{M}^{G}\left(\phi_{\lambda}, P, a\right)$ and $m_{M}^{G, \mathcal{E}}\left(\phi_{\lambda}^{\prime}, P, a\right)$ both vanish if $M$ is not a maximal Levi subgroup. Assume therefore that $M$ is maximal. Then $m_{M}^{G}\left(\phi_{\lambda}, P, a\right)$ is a logarithmic derivative of the function $m_{a}\left(\phi_{\lambda}\right)$ (relative to the coordinate $\frac{1}{2} a(\lambda)$ ). Since logarithmic derivatives transform products to sums, the formula (5.6) gives rise to an identity

$$
m_{M}^{G}\left(\phi_{\lambda}, P, a\right)=\sum_{G^{\prime} \in \mathcal{E}_{M^{\prime}}(G) / \widehat{Z}_{a}} m_{\tilde{M}^{\prime}}^{\tilde{G}^{\prime}}\left(\phi_{\lambda}^{\prime}, P^{\prime}, a^{\prime}\right) .
$$

The essential point is to show that the right hand side of this identity matches the right hand side of the identity

$$
m_{M}^{G, \mathcal{E}}\left(\phi_{\lambda}^{\prime}, P, a\right)=\sum_{G^{\prime} \in \mathcal{E}_{M^{\prime}}(G)} \iota_{M^{\prime}}\left(G, G^{\prime}\right) n_{\tilde{M}^{\prime}}^{\tilde{G}^{\prime}}\left(\phi_{\lambda}^{\prime}, P^{\prime}, a^{\prime}\right)
$$

in which the explicit formula

$$
n_{\tilde{M}^{\prime}}^{\tilde{G}^{\prime}}\left(\phi_{\lambda}^{\prime}, P^{\prime}, a^{\prime}\right)=\left|\widehat{Z}_{a^{\prime}} / \widehat{Z}_{a^{\prime}} \cap Z\left(\widehat{\widetilde{G}}^{\prime}\right)^{\Gamma}\right|^{-1} m_{\tilde{M}^{\prime}}^{\tilde{G}^{\prime}}\left(\phi_{\lambda}^{\prime}, P^{\prime}, a^{\prime}\right)
$$

holds. The case that $M^{\prime}=M^{*}$ is of course to be included here. This forces the explicit formula to be compatible with the inductive definition of $n_{M}^{G}\left(\phi_{\lambda}, P, a\right)$ above, and to be thus valid for each $\left(\widetilde{G}^{\prime}, \widetilde{M}^{\prime}\right)$. The general argument is identical to that of [A10, pp. 1145-1146]. (It is also reminiscent of a part of the proof of Lemma 4.1 from the last section.) One shows that

$$
\iota_{M^{\prime}}\left(G, G^{\prime}\right) n_{\tilde{M}^{\prime}}^{\tilde{G}^{\prime}}\left(\phi_{\lambda}^{\prime}, P^{\prime}, a^{\prime}\right)=\left|\widehat{Z}_{a} / \widehat{Z}_{a} \cap Z(\widehat{G})^{\Gamma}\right|^{-1} m_{\tilde{M}^{\prime}}^{\tilde{G}^{\prime}}\left(\phi_{\lambda}^{\prime}, P^{\prime}, a^{\prime}\right),
$$

by a simple comparison of the relevant coefficients. (In the analogue of this formula on p. 1146 of A10, the intersection $\widehat{Z}_{a} \cap Z(\widehat{G})^{\Gamma}$ was mistakenly written as $\widehat{Z}_{a} \cap Z\left(\widehat{G}^{\prime}\right)^{\Gamma}$, or rather $Z_{a} \cap Z\left(\widehat{G}^{\prime}\right)^{\Gamma}$, since $\widehat{Z}_{a}$ was denoted by $Z_{a}$ in A10.) This establishes that $m_{M}^{G}\left(\phi_{\lambda}, P, a\right)$ equals $m_{M}^{G, \mathcal{E}}\left(\phi_{\lambda}^{\prime}, P, a\right)$.

With the required identity established in the case that $A$ consists of one element $a$, we apply the standard splitting formula for $(G, M)$-families to prove it inductively for general $A$. The argument is identical to that in [A10, pp. 1146-1147]. It allows us 
to conclude that $m_{M}^{G}\left(\phi_{\lambda}, P, A\right)$ equals $m_{M}^{G, \mathcal{E}}\left(\phi_{\lambda}^{\prime}, P, A\right)$ for any $A$. Taking $A$ equal to $\Sigma(\widehat{\bar{P}})$, we then obtain the required result that $m_{M}^{G}\left(\phi_{\lambda}, P\right)$ equals $m_{M}^{G, \mathcal{E}}\left(\phi_{\lambda}^{\prime}, P\right)$.

We will now be able to stabilize the linear form $I_{M}^{P}(\gamma, f)$ on the Hecke algebra $\mathcal{H}(G, \zeta)$. The objects $M^{\prime}$ and $T^{\prime}$ are fixed, while $\widetilde{T}^{\prime}$ is the extension of $T^{\prime}$ attached to the auxiliary datum $\left(\widetilde{M}^{\prime}, \widetilde{\xi}_{M}^{\prime}\right)$ that comes with the transfer factor $\Delta_{M}$. The first ingredient in the stabilization of $I_{M}^{P}(\gamma, f)$ is the function

$$
I_{M}^{P}\left(\sigma^{\prime}, f\right)=\sum_{\gamma \in \Gamma_{G-\text { reg }}(M)} \Delta_{M}\left(\sigma^{\prime}, \gamma\right) I_{M}^{P}(\gamma, f)
$$

of $\sigma^{\prime} \in \widetilde{T}_{G \text {-reg }}^{\prime}(\mathbb{R})$ that is analogous to (1.4). Then $I_{M}^{P}\left(\sigma^{\prime}, f\right)$ equals the integral over $\tau \in T_{\varepsilon_{P}}(M, \zeta)$ of the product of

$$
\sum_{\gamma} \Delta_{M}\left(\sigma^{\prime}, \gamma\right) \theta_{M}(\gamma, \tau)
$$

with

$$
m_{M}(\tau, P) f_{M}(\tau) .
$$

In [S3, §4-5], Shelstad establishes a spectral theory of endoscopy that is dual to the geometric theory she had developed earlier. We shall briefly review the results here, in the context of the $K$-group $G$ and our discussion at the beginning of the section.

Suppose that $\phi$ belongs to $\Phi_{\text {temp }}(M, \zeta)$. Then the linear form

$$
h^{M}(\phi)=\sum_{\tau \in T_{\phi}} h_{M}(\tau), \quad h \in \mathcal{C}(M, \zeta),
$$

on $\mathcal{C}(M, \zeta)$ is a stable distribution, called the stable character of $\phi$. In case $G$ is quasisplit, it attaches a continuous linear form

$$
h^{*}\left(\phi^{*}\right)=h^{M}(\phi), \quad h^{*} \in \mathcal{S}\left(M^{*}, \zeta^{*}\right),
$$

on $\mathcal{S}\left(M^{*}, \zeta^{*}\right)$ to every parameter $\phi^{*} \in \Phi_{\text {temp }}\left(M^{*}, \zeta^{*}\right)$, since there is a bijection $\phi \rightarrow \phi^{*}$ from $\Phi_{\text {temp }}(M, \zeta)$ to $\Phi_{\text {temp }}\left(M^{*}, \zeta^{*}\right)$. Assume that $G$ is general, but that $\phi$ is the image in $\Phi_{\text {temp }}(M, \zeta)$ of a parameter $\phi^{\prime} \in \Phi_{\text {temp }}\left(\widetilde{M}^{\prime}, \widetilde{\zeta}^{\prime}\right)$. Then the mapping

$$
h \longrightarrow h^{\tilde{M}^{\prime}}\left(\phi^{\prime}\right), \quad h \in \mathcal{C}(M, \zeta),
$$

is an invariant, tempered distribution on $M(\mathbb{R})$. Shelstad shows that it is a linear combination of characters of representations in the packet $\Pi_{\phi}$. It is therefore a linear combination of virtual characters in the packet $T_{\phi}$. In other words

$$
h^{M^{\prime}}\left(\phi^{\prime}\right)=\sum_{\tau \in T_{\phi}} \Delta_{M}\left(\phi^{\prime}, \tau\right) h_{M}(\tau)
$$

for coefficients $\Delta_{M}\left(\sigma^{\prime}, \tau\right)$ that depend on the transfer factor $\Delta_{M}$.

It follows from the existence of the function $\theta_{M}(\gamma, \tau)$, together with the results of Shelstad, that we can write

$$
h^{M}(\sigma)=\int_{\Phi_{\mathrm{temp}}(M, \zeta)} \eta_{M}(\sigma, \phi) h^{M}(\phi) d \phi, \quad \sigma \in T_{\mathrm{reg}}(\mathbb{R}), h \in \mathcal{C}(M, \zeta),
$$

for a smooth function

$$
\eta_{M}(\sigma, \phi), \quad \sigma \in T_{\text {reg }}(\mathbb{R}), \phi \in \Phi_{\text {temp }}(M, \zeta) .
$$


This function satisfies

$$
\eta_{M}\left(\sigma, \phi_{\lambda}\right)=e^{-\lambda\left(H_{M}(\sigma)\right)} \eta_{M}(\sigma, \phi), \quad \lambda \in i \mathfrak{a}_{M, Z}^{*} .
$$

It therefore continues analytically to a tempered function of $\phi$ in the space $\Phi_{\varepsilon_{P}}(M, \zeta)$. The integral can consequently be deformed from $\Phi_{\text {temp }}(M, \zeta)$ to $\Phi_{\varepsilon_{P}}(M, \zeta)$, if we take $h$ to be in the Hecke algebra $\mathcal{H}(M, \zeta)$. We shall write

$$
\eta_{M}^{\prime}\left(\sigma^{\prime}, \phi^{\prime}\right)=\eta_{\tilde{M}^{\prime}}\left(\sigma^{\prime}, \phi^{\prime}\right)
$$

for the analogue of $\eta_{M}(\sigma, \phi)$ for $\tilde{M}^{\prime}$.

We claim that the sum (5.8) in the formula for $I_{M}^{P}\left(\sigma^{\prime}, f\right)$ equals

$$
\sum_{\phi^{\prime} \in \Phi_{\varepsilon_{P}}\left(\tilde{M}^{\prime}, \tilde{\zeta}^{\prime}\right)} \eta_{M}^{\prime}\left(\sigma^{\prime}, \phi^{\prime}\right) \Delta_{M}\left(\phi^{\prime}, \tau\right) .
$$

To see this, we need only integrate the two functions of $\tau \in T_{\varepsilon_{P}}(M, \zeta)$ against an arbitrary function $a_{M}(\tau)$ in $I \mathcal{H}(M, \zeta)$, and then observe that the resulting integrals are equal by the definitions above. It follows that

$$
\begin{aligned}
& I_{M}^{P}\left(\sigma^{\prime}, f\right) \\
& =\int_{T_{\varepsilon_{P}}(M, \zeta)}\left(\sum_{\phi^{\prime}} \eta_{M}^{\prime}\left(\sigma^{\prime}, \phi^{\prime}\right) \Delta_{M}\left(\phi^{\prime}, \tau\right)\right) m_{M}(\tau, P) f_{M}(\tau) d \tau \\
& =\int_{\Phi_{\varepsilon_{P}}(M, \zeta)} \sum_{\phi^{\prime}} \eta_{M}^{\prime}\left(\sigma^{\prime}, \phi^{\prime}\right) m_{M}(\phi, P) f^{\tilde{M}^{\prime}}\left(\phi^{\prime}\right) d \phi,
\end{aligned}
$$

since the function $m_{M}(\phi, P)=m_{M}(\tau, P)$ depends only on $\phi$. The last sum over $\phi^{\prime}$ is understood to be taken over the preimage of $\phi$ in $\Phi_{\varepsilon_{P}}\left(\widetilde{M}^{\prime}, \widetilde{\zeta}^{\prime}\right)$. It can be combined with the integral over $\Phi_{\varepsilon_{P}}(M, \zeta)$ to give an integral

$$
\int_{\Phi_{\varepsilon_{P}}\left(\tilde{M}^{\prime}, \tilde{\zeta}^{\prime}\right)} \eta_{M}^{\prime}\left(\sigma^{\prime}, \phi^{\prime}\right) m_{M}(\phi, P) f^{\tilde{M}^{\prime}}\left(\phi^{\prime}\right) d \phi^{\prime}
$$

over $\Phi_{\varepsilon_{P}}\left(\widetilde{M}^{\prime}, \widetilde{\zeta}^{\prime}\right)$. Substituting the formula (5.4) of the proposition into this expression, we find that $I_{M}^{P}\left(\sigma^{\prime}, f\right)$ equals

$$
\int_{\Phi_{\varepsilon_{P}}\left(\tilde{M}^{\prime}, \tilde{\zeta}^{\prime}\right)} \eta_{M}^{\prime}\left(\sigma^{\prime}, \phi^{\prime}\right) \sum_{G^{\prime} \in \mathcal{E}_{M^{\prime}}(G)} \iota_{M^{\prime}}\left(G, G^{\prime}\right) n_{\tilde{M}^{\prime}}^{\tilde{G}^{\prime}}\left(\phi^{\prime}, P^{\prime}\right) f^{\tilde{M}^{\prime}}\left(\phi^{\prime}\right) d \phi^{\prime} .
$$

If $G$ is quasisplit, we define

$$
S_{M}^{G, P}(\sigma, f)=\int_{\Phi_{\varepsilon_{P}}(M, \zeta)} \eta_{M}(\sigma, \phi) n_{M}^{G}(\phi, P) f^{M}(\phi) d \phi
$$

and

$$
\widehat{S}_{M^{*}}^{G^{*} P^{*}}\left(\sigma^{*}, f^{*}\right)=S_{M}^{G, P}(\sigma, f),
$$

for any point $\sigma \in T_{G \text {-reg }}(\mathbb{R})$ with image $\sigma^{*} \in T_{G \text {-reg }}^{*}(\mathbb{R})$ in $G^{*}(\mathbb{R})$. The linear form $S_{M}^{G, P}(\sigma, f)$ on $\mathcal{H}(G, \zeta)$ is stable, so the last definition here makes sense. Applying it to $\widetilde{G}^{\prime}, \widetilde{M}^{\prime}, \widetilde{P}^{\prime}$ and $\sigma^{\prime}$, where $\widetilde{P}^{\prime}$ is the preimage of $P^{\prime}$ in $\mathcal{P}^{\tilde{G}^{\prime}}\left(\widetilde{M}^{\prime}\right)$, we conclude that

$$
I_{M}^{P}\left(\sigma^{\prime}, f\right)=\sum_{G^{\prime} \in \mathcal{E}_{M^{\prime}}(G)} \iota_{M^{\prime}}\left(G, G^{\prime}\right) \widehat{S}_{\tilde{M}^{\prime}}^{\tilde{G}^{\prime}, \tilde{P}^{\prime}}\left(\sigma^{\prime}, f^{\prime}\right) .
$$


This formula represents a stabilization of the right hand side of (5.1). It amounts to a single assertion that combines the definitions of $\S 1$, but with $I_{M}^{P}(\gamma, f)$ in place of $I_{M}(\gamma, f)$ and $f$ taken to be function in $\mathcal{H}(G, \zeta)$, with the corresponding assertions from Theorem 1.1.

To exploit (5.11), we shall also need to stabilize the left hand side of (5.1). We begin by noting that any character on $A_{M}(\mathbb{R})^{0}$ can be lifted to a character on $A_{\tilde{M}^{\prime}}(\mathbb{R})^{0}$, since $A_{\tilde{M}^{\prime}}(\mathbb{R})^{0}$ is isomorphic to the additive group of the real vector space $\mathfrak{a}_{\tilde{M}^{\prime}}$. It follows from [LS1, (4.4)] that we can choose the representative data $\left(\widetilde{M}^{\prime}, \widetilde{\xi}_{M}^{\prime}\right)$ and $\Delta_{M}$ for $M^{\prime}$ so that

$$
\Delta_{M}\left(\sigma^{\prime} a^{\prime}, \gamma a\right)=\Delta_{M}\left(\sigma^{\prime}, \gamma\right), \quad a^{\prime} \in A_{\tilde{M}^{\prime}}(\mathbb{R})^{0},
$$

where $a$ is the image of $a^{\prime}$ in $A_{M}(\mathbb{R})^{0}$. If

$$
\sigma_{T}^{\prime}=\sigma^{\prime} \exp T, \quad T \in \mathfrak{a}_{\tilde{M}^{\prime}},
$$

the distribution $I_{M}\left(\sigma_{T}^{\prime}, \cdot\right)$ then depends only on the image of $T$ in the quotient $\mathfrak{a}_{M}$ of $\mathfrak{a}_{\tilde{M}^{\prime}}$ (which we continue to denote by $T$ ). It follows from (5.1) that

$$
\lim _{T \underset{P, r}{\longrightarrow} \infty} I_{M}\left(\sigma_{T}^{\prime}, f_{T}\right)=I_{M}^{P}\left(\sigma^{\prime}, f\right) .
$$

Incidentally, the original limit (5.1) was shown in A14 to be uniform for $\gamma$ in any relatively compact subset $\Gamma$ of $T_{G \text {-reg }}(\mathbb{R})$. It follows that the limit (5.13) is uniform for $\sigma^{\prime}$ in any relatively compact subset $\Gamma^{\prime}$ of $\widetilde{T}_{G \text {-reg }}^{\prime}(\mathbb{R})$.

The function $f_{T}$ represents the image of $f$ under the Schwartz multiplier $\alpha_{T}$ introduced in [A14, §1]. To help us understand its transfer, we shall say a word about the transfer of general multipliers.

The notion of a Schwartz multiplier in [A14, §1] extends in a natural way to the space $\mathcal{C}(G, \zeta)$ of this paper. A Schwartz multiplier for $\mathcal{C}(G, \zeta)$ is an endomorphism

$$
\alpha: f=\bigoplus_{\iota} f \longrightarrow f_{\alpha}=\bigoplus_{\iota} f_{\iota, \alpha_{\iota}}, \quad f \in \mathcal{C}(G, \zeta),
$$

where for each $\iota \in \pi_{0}(G), f_{\iota} \rightarrow f_{\iota, \alpha_{\iota}}$ is a continuous endomorphism of $\mathcal{C}\left(G_{\iota}, \zeta_{\iota}\right)$ that commutes with left and right translation. It is characterized by the property

$$
\pi\left(f_{\alpha}\right)=\widehat{\alpha}(\pi) \pi(f), \quad \pi \in \Pi_{\text {temp }}(G, \zeta), f \in \mathcal{C}(G, \zeta),
$$

where $\widehat{\alpha}$ is a smooth complex valued function on $\Pi_{\text {temp }}(G, \zeta)$ of which any invariant derivative is slowing increasing, and whose value $\widehat{\alpha}(\pi)$ depends only on the induced tempered cuspidal representation of which $\pi$ is a constituent. (See [A14, p. 171].) We can identify $\widehat{\alpha}$ with a function $\widehat{\alpha}(\tau)$ on $T_{\text {temp }}(G, \zeta)$, thereby treating $\alpha$ as a multiplier on the invariant Schwartz space $\mathcal{I}(G, \zeta)$. We write $\mathcal{M}(G, \zeta)$ for the algebra of multipliers on $\mathcal{C}(G, \zeta)$.

We shall say that a multiplier $\alpha \in \mathcal{M}(G, \zeta)$ is stable if its value $\widehat{\alpha}(\tau)$ depends only on the $L$-packet of $\tau$. With this condition, $\alpha$ can be identified with a smooth function

$$
\widehat{\alpha}(\phi)=\widehat{\alpha}(\tau), \quad \phi \in \Phi_{\text {temp }}(G, \zeta), \tau \in T_{\phi},
$$

on $\Phi_{\text {temp }}(G, \zeta)$. Suppose that $G^{\prime}$ is an endoscopic datum for $G$, with transfer factor $\Delta_{G}$, and that $\phi^{\prime} \in \Phi_{\text {temp }}\left(\widetilde{G}^{\prime}, \widetilde{\zeta}^{\prime}\right)$ maps to a parameter $\phi \in \Phi_{\text {temp }}(G, \zeta)$. Then if $\alpha$ 
is a Schwartz multiplier, we see that

$$
\begin{aligned}
\left(f_{\alpha}\right)^{\prime}\left(\phi^{\prime}\right) & =\sum_{\tau \in T_{\phi}} \Delta_{G}\left(\phi^{\prime}, \tau\right)\left(f_{\alpha}\right)_{G}(\tau) \\
& =\sum_{\tau \in T_{\phi}} \Delta_{G}\left(\phi^{\prime}, \tau\right) \widehat{\alpha}(\tau) f_{G}(\tau)=\widehat{\alpha}(\phi) f^{\prime}\left(\phi^{\prime}\right) .
\end{aligned}
$$

It follows that

$$
\left(f_{\alpha}\right)^{\prime}=\left(f^{\prime}\right)_{\alpha^{\prime}},
$$

where $\alpha^{\prime}$ is the stable multiplier for $\left(\widetilde{G}^{\prime}, \widetilde{\zeta}^{\prime}\right)$ defined by setting

$$
\widehat{\alpha}^{\prime}\left(\phi^{\prime}\right)=\widehat{\alpha}(\phi), \quad \phi^{\prime} \in \Phi_{\text {temp }}\left(\widetilde{G}^{\prime}, \widetilde{\zeta}^{\prime}\right),
$$

for the image $\phi$ of $\phi^{\prime}$ in $\Phi_{\text {temp }}(G, \zeta)$. We write $S \mathcal{M}(G, \zeta)$ for the subalgebra of stable multipliers in $\mathcal{M}(G, \zeta)$.

We can regard the multiplier $\alpha_{T}$ that gives rise to the function $f_{T}=f_{\alpha_{T}}$ in $(5.1)$ as an element in $\mathcal{M}(G, \zeta)$. It is given by a double sum

$$
\alpha_{T}=\sum_{\{L\}} \sum_{u \in U(M, L)} \alpha^{u T},
$$

in the notation of [A14, §1]. More concretely, we have

$$
\widehat{\alpha}_{T}\left(\pi^{G}\right)=\sum_{u \in U(M, L)} e^{\nu_{\pi}(u T)}, \quad \pi \in \Pi_{\mathrm{temp}, \operatorname{cusp}}(L, \zeta),
$$

where $\pi^{G}=\mathcal{I}_{Q}(\pi)$ is the induced representation, and $\nu_{\pi}$ is the imaginary part of the infinitesimal character of $\pi$. Since it is defined in terms of the infinitesimal character, $\alpha_{T}$ is stable. The same goes for the multiplier $\alpha^{S}$ attached in [A14, $\left.\S 1\right]$ to any point $S \in \mathfrak{a}_{L}$. Notice that

$$
f_{T+Z}=\zeta(\exp Z) f_{T}, \quad Z \in \mathfrak{a}_{Z}
$$

It follows that the function $I_{M}\left(\sigma_{T}^{\prime}, f_{T}\right)$ in (5.1) depends only on the image of $T$ in $\mathfrak{a}_{M} / \mathfrak{a}_{Z}$.

To study the stabilization of the left hand side of (5.1), we fix a relatively compact subset $\Gamma^{\prime}$ of $\widetilde{T}_{G \text {-reg }}^{\prime}(\mathbb{R})$. For the moment, we may as well take $f \in \mathcal{C}(G, \zeta)$ to be a general Schwartz function. Beginning with the usual argument, we write the difference

$$
I_{M}^{\mathcal{E}}\left(\sigma_{T}^{\prime}, f_{T}\right)-\varepsilon(G) S_{M}^{G}\left(M^{\prime}, \sigma_{T}^{\prime}, f_{T}\right)
$$

as a sum

$$
\sum_{G^{\prime} \in \mathcal{E}_{M^{\prime}}^{0}(G)} \iota_{M^{\prime}}\left(G, G^{\prime}\right) \widehat{S}_{\tilde{M}^{\prime}}^{\tilde{G}^{\prime}}\left(\sigma_{T}^{\prime}, f_{T}^{\prime}\right)
$$

We recall from the proof of Lemma 4.1 that $\varepsilon(G)=0$ unless $G$ is quasisplit, in which case $\varepsilon(G)=1$. The function $f_{T}^{\prime}=\left(f_{T}\right)^{\prime}=\left(f_{\alpha_{T}}\right)^{\prime}$ here equals the image of $f^{\prime}$ under the multiplier $\left(\alpha_{T}\right)^{\prime}$ in $\operatorname{SM}\left(\widetilde{G}^{\prime}, \widetilde{\zeta}^{\prime}\right)$. However, $\left(\alpha_{T}\right)^{\prime}$ is not generally equal to the multiplier $\left(\alpha^{\prime}\right)_{T}$. In other words, $f_{T}^{\prime}$ need not equal $\left(f^{\prime}\right)_{T}$. The following lemma tells us that this discrepancy is not serious. 
Lemma 5.2. For any $G^{\prime} \in \mathcal{E}_{M^{\prime}}^{0}(G)$, we have

$$
\lim _{T \underset{P, r}{\longrightarrow} \infty}\left(\widehat{S}_{\tilde{M}^{\prime}}^{\tilde{G}^{\prime}}\left(\sigma_{T}^{\prime}, f_{T}^{\prime}\right)-\widehat{S}_{\tilde{M}^{\prime}}^{\tilde{G}^{\prime}}\left(\sigma_{T}^{\prime},\left(f^{\prime}\right)_{T}\right)\right)=0
$$

uniformly for $\sigma^{\prime} \in \Gamma^{\prime}$.

Proof. We assume that the implicit transfer factor $\Delta_{G}$ that defines $f^{\prime}$ satisfies the obvious analogue for $G$ of (5.12). The terms in (5.18) are then well defined functions of $T \in \mathfrak{a}_{M} / \mathfrak{a}_{Z}$.

Consider a $G$-relevant parameter $\phi^{\prime} \in \Phi_{\text {temp }}\left(\widetilde{G}^{\prime}, \widetilde{\zeta}^{\prime}\right)$ for $\widetilde{G}^{\prime}$, with image $\phi$ in $\Phi_{\text {temp }}(G, \zeta)$. Then $\phi^{\prime}$ and $\phi$ are induced respectively from cuspidal parameters $\phi_{1}^{\prime} \in \Phi_{\text {temp,cusp }}\left(\widetilde{L}_{1}^{\prime}, \widetilde{\zeta}_{1}\right)$ and $\phi_{1} \in \Phi_{\text {temp,cusp }}\left(M_{1}, \zeta\right)$, for Levi subgroups $L_{1}^{\prime} \subset G^{\prime}$ and $M_{1} \subset G$. We have

$$
\begin{aligned}
\left(f_{T}^{\prime}\right)\left(\phi^{\prime}\right) & =\left(f_{\alpha_{T}}\right)^{\prime}\left(\phi^{\prime}\right) \\
& =\left(\alpha_{T}\right)^{\prime}\left(\phi^{\prime}\right) f^{\prime}\left(\sigma^{\prime}\right)=\alpha_{T}(\phi) f^{\prime}\left(\phi^{\prime}\right) \\
& =\left(\sum_{u \in U\left(M, M_{1}\right)} e^{\nu_{1}(u T)}\right) f^{\prime}\left(\phi^{\prime}\right),
\end{aligned}
$$

where $\nu_{1} \in i \mathfrak{a}_{M_{1}}^{*}$ represents the imaginary part of the infinitesimal character of $\phi_{1}$. For the given $L_{1}^{\prime}$ and $M_{1}$, we fix an admissible embedding $\mathfrak{a}_{L_{1}^{\prime}} \hookrightarrow \mathfrak{a}_{M_{1}}$. By this, we mean the injection attached to an admissible embedding of a maximal torus of $L_{1}^{\prime}$ over $\mathbb{R}$ into $G$ that takes $A_{L_{1}^{\prime}}$ into $A_{M_{1}}$. It is a consequence of the construction (and the condition above on $\Delta_{G}$ ) that we can choose the embedding so that $\nu_{1}$ lies in the subspace $i \mathfrak{a}_{L_{1}^{\prime}}^{*}$ of $i \mathfrak{a}_{M_{1}}^{*}$, thereby representing the infinitesimal character of $\phi^{\prime}$. We then have

$$
\begin{aligned}
\left(f^{\prime}\right)_{T}\left(\phi^{\prime}\right) & =\left(f^{\prime}\right)_{\left(\alpha^{\prime}\right)_{T}\left(\phi^{\prime}\right)} \\
& =\left(\sum_{u^{\prime} \in U^{\prime}\left(M^{\prime}, L_{1}^{\prime}\right)} e^{\nu_{1}\left(u^{\prime} T\right)}\right) f^{\prime}\left(\phi^{\prime}\right) .
\end{aligned}
$$

We have written $U^{\prime}\left(M^{\prime}, L_{1}^{\prime}\right)=U^{G^{\prime}}\left(M^{\prime}, L_{1}^{\prime}\right)$ here for the set of embeddings from $\mathfrak{a}_{M^{\prime}}$ into $\mathfrak{a}_{L_{1}^{\prime}}$ induced by the adjoint action of $G^{\prime}$. This set comes with embeddings

$$
U^{\prime}\left(M^{\prime}, L_{1}^{\prime}\right) \subset U\left(M, L_{1}\right) \subset U\left(M, M_{1}\right),
$$

where $U\left(M, L_{1}\right)=U^{G}\left(M, L_{1}\right)$ is the associated set of embeddings of $\mathfrak{a}_{M} \cong \mathfrak{a}_{M^{\prime}}$ into $\mathfrak{a}_{L_{1}} \cong \mathfrak{a}_{L_{1}^{\prime}}$ attached to $G$, and $U\left(M, M_{1}\right)$ is the larger set that indexes the earlier sum. (We have written $L_{1} \in \mathcal{L}\left(M_{1}\right)$ here for the Levi subgroup of $G$ corresponding to the subspace $\mathfrak{a}_{L_{1}^{\prime}}$ of $\mathfrak{a}_{M_{1}}$.) We conclude that

$$
f_{T}^{\prime}\left(\phi^{\prime}\right)-\left(f^{\prime}\right)_{T}\left(\phi^{\prime}\right)=\alpha_{T, L_{1}^{\prime}, M_{1}}^{\prime}\left(\phi^{\prime}\right) f^{\prime}\left(\phi^{\prime}\right),
$$

where

$$
\alpha_{T, L_{1}^{\prime}, M_{1}}^{\prime}\left(\phi^{\prime}\right)=\sum_{u \in U\left(M, M_{1}\right)-U^{\prime}\left(M^{\prime}, L_{1}^{\prime}\right)} e^{\nu_{1}(u T)} .
$$

The pair $\left(L_{1}^{\prime}, M_{1}\right)$ is not uniquely determined by $\phi^{\prime}$. The correspondence that assigns any such pair to a given $\phi^{\prime}$ gives rise to a mapping

$$
\phi^{\prime} \longrightarrow\left\{\left(L_{1}^{\prime}, M_{1}\right)\right\}
$$

from the set of $G$-relevant parameters in $\Phi_{\text {temp }}\left(\widetilde{G}^{\prime}, \widetilde{\zeta}^{\prime}\right)$ onto a finite set of equivalence classes of pairs. It is not hard to see that $\alpha_{T, L_{1}^{\prime}, M_{1}}^{\prime}\left(\phi^{\prime}\right)$ depends only on the 
equivalence class $\left\{\left(L_{1}^{\prime}, M_{1}\right)\right\}$ of $\left(L_{1}^{\prime}, M_{1}\right)$. This function represents a stable multiplier $\alpha_{T, L_{1}^{\prime}, M_{1}}^{\prime}$ in $S \mathcal{M}\left(\widetilde{G}^{\prime}, \widetilde{\zeta}^{\prime}\right)$, whose value at any $\phi^{\prime} \in \Phi_{\text {temp }}\left(\widetilde{G}^{\prime}, \widetilde{\zeta}^{\prime}\right)$ equals the finite sum (5.19) if $\phi^{\prime}$ maps to $\left\{\left(L_{1}^{\prime}, M_{1}\right)\right\}$, and equals 0 otherwise. It follows that

$$
f_{T}^{\prime}-\left(f^{\prime}\right)_{T}=\sum_{\left\{\left(L_{1}^{\prime}, M_{1}\right)\right\}} f_{T, L_{1}^{\prime}, M_{1}}^{\prime},
$$

where $f_{T, L_{1}^{\prime}, M_{1}}^{\prime}$ denotes the transform of $f^{\prime}$ by the multiplier $\alpha_{T, L_{1}^{\prime}, M_{1}}^{\prime}$. Observe that any summand on the right hand side of (5.19) satisfies

$$
e^{\nu_{1}(u T)}=e^{\nu_{1}\left((u T)_{1}^{\prime}\right)},
$$

where $(u T)_{1}^{\prime}$ is the projection of the point $u T \in \mathfrak{a}_{M_{1}}$ onto $\mathfrak{a}_{L_{1}}$. Using the definitions (5.19) and [A14, (1.12)], it is then not hard to show that

$$
f_{T, L_{1}^{\prime}, M_{1}}^{\prime}=\sum_{u}\left(f^{\prime}\right)_{\alpha^{S^{\prime}}}=\sum_{u}\left(f^{\prime}\right)^{S^{\prime}}, \quad S^{\prime}=(u T)_{1}^{\prime},
$$

where the sums are each taken over the complement of $U^{\prime}\left(M^{\prime}, L_{1}^{\prime}\right)$ in $U\left(M, M_{1}\right)$.

To complete the proof, we must show that the contribution to the limit (5.18) of a summand $f_{T, L_{1}^{\prime}, M_{1}}^{\prime}$ in (5.20) vanishes. This requires another lemma. We need the stable analogue of an important estimate (5.16) from A14, which applies to the case that $G$ is quasisplit. We state it in terms of a minimal Levi subgroup $M_{0} \subset M$, with minimal parabolic subgroup $P_{0} \in \mathcal{P}\left(M_{0}\right)$ for which both $M$ and a second given Levi subgroup $M_{1}$ are standard. The lemma pertains to an open cone $c_{0}=c_{0}^{+}$in $\mathfrak{a}_{0}^{+}=\mathfrak{a}_{P_{0}}^{+}$, points $S \in \mathfrak{a}_{M_{1}}$ and $T \in \mathfrak{a}_{P}^{+}$such that $T$ is $\left(c_{0}, S\right)$-dominant, and the associated distance function $d_{c_{0}}(T, S)$, all introduced in the preamble to Lemma 4.4 of A14.

Lemma 5.3. Assume that $G$ is quasisplit, and that $\Gamma$ is a relatively compact subset of $T_{G \text {-reg }}(\mathbb{R})$. Then for any $n \geq 0$, there is a continuous seminorm $\|\cdot\|_{n}$ on $\mathcal{C}(G, \zeta)$ such that

$$
\left|S_{M}^{G}\left(\sigma_{T}, f^{S}\right)\right| \leq\|f\|_{n}\left(1+d_{c_{0}}(T, S)\right)^{-n},
$$

for any $\sigma \in \Gamma, f \in \mathcal{C}(G, \zeta), T \in \mathfrak{a}_{P}^{+}$and $S \in \mathfrak{a}_{M_{1}}$ such that $T$ is $\left(c_{0}, S\right)$-dominant.

Proof. The derivation of (5.22) from the inequality [A14, (5.16)] is similar in principle to the argument A14, Corollary 5.2] by which the earlier inequality was deduced from its noninvariant analogue [A14, (5.15)]. It is an inductive proof, with the mappings $f \rightarrow f^{\prime}$ taking the place of the earlier mappings $f \rightarrow \phi_{L}(f)$.

By definition, $S_{M}^{G}\left(\sigma_{T}, f^{S}\right)$ equals

$$
I_{M}\left(\sigma_{T}, f^{S}\right)-\sum_{G^{\prime} \in \mathcal{E}_{M^{\prime}}^{0}(G)} \iota_{M^{\prime}}\left(G, G^{\prime}\right) \widehat{S}_{\tilde{M}^{\prime}}^{\tilde{G}^{\prime}}\left(\sigma_{T}^{\prime},\left(f^{S}\right)^{\prime}\right), \quad M^{\prime}=M^{*} .
$$

The analogue of $(5.22)$ for $I_{M}\left(\sigma_{T}, f^{S}\right)$ follows from (1.4) and the estimate A14, (5.16)] for $I_{M}\left(\gamma_{T}, f^{S}\right)$. To estimate the summands, we need to say something about the function

$$
\left(f^{S}\right)^{\prime}=\left(f_{\alpha} s\right)^{\prime}=\left(f^{\prime}\right)_{\left(\alpha^{S}\right)^{\prime}}, \quad G^{\prime} \in \mathcal{E}_{M^{*}}^{0}(G) .
$$

The argument at this stage becomes a little more elaborate than that of A14. However, the complications can be treated as a special case of the discussion above 
that led to the decomposition (5.20). It follows easily from this discussion that there is a decomposition

$$
\left(f^{S}\right)^{\prime}=\left|W\left(M_{1}\right)\right|^{-1} \sum_{w \in W\left(M_{1}\right)} \sum_{i}\left(f^{\prime}\right)^{(w S)_{i}^{\prime}}
$$

for any given $G^{\prime} \in \mathcal{E}_{M^{*}}^{0}(G)$, where $i$ indexes a finite set of Levi subgroups $\left\{L_{i}^{\prime}\right\}$ of $G^{\prime}$, with admissible embeddings $\mathfrak{a}_{L_{i}^{\prime}} \subset \mathfrak{a}_{M_{1}}$, and $(w S)_{i}^{\prime}$ is the projection of $w S$ onto the subspace $\mathfrak{a}_{L_{i}^{\prime}}$ of $\mathfrak{a}_{M_{1}}$. This is the analogue of the decomposition [A14, (5.17)] from the proof of [A14, Corollary 5.2]. We are assuming that $G$ is quasisplit and that $M^{*}=M^{\prime}$ is a Levi subgroup of $G^{\prime}$. We can consequently fix a minimal Levi subgroup $M_{0}^{*}=M_{0}^{\prime}$ of $G^{\prime}$ that is at the same time a quasisplit inner form of $M_{0}$. We take $P_{0}^{\prime} \in \mathcal{P}\left(M_{0}^{\prime}\right)$ to be the minimal parabolic subgroup of $G^{\prime}$ whose chamber $\mathfrak{a}_{P_{0}^{\prime}}^{+}$in the space $\mathfrak{a}_{M_{0}^{\prime}}=\mathfrak{a}_{M_{0}}$ contains the chamber $\mathfrak{a}_{0}^{+}=\mathfrak{a}_{P_{0}}^{+}$, and hence also the open cone $c_{0}$. We are free to choose the Levi subgroups $L_{i}^{\prime}$ of $G^{\prime}$ to be standard with respect to $P_{0}^{\prime}$. For any $i$, and any element $w^{\prime}$ in the Weyl group $W\left(M_{0}^{\prime}\right)$, $w^{\prime}(w S)_{i}^{\prime}$ is easily seen to belong to the convex hull of $W\left(M_{0}\right) S$ in $\mathfrak{a}_{M_{0}}$. It follows from the definitions in [A14, §4] that if $T$ is $\left(c_{0}, S\right)$-dominant (relative to $G$ ), it is also $\left(c_{0},(w S)_{i}^{\prime}\right)$-dominant (relative to $\left.G^{\prime}\right)$. Moreover, the corresponding distance functions satisfy

$$
d_{c_{0}}(T, S)=d_{c_{0}}^{G}(T, S) \leq d_{c_{0}}^{\tilde{G}^{\prime}}\left(T,(w S)_{i}^{\prime}\right) .
$$

We assume inductively that the analogue of (5.22) holds for each group $G^{\prime} \in$ $\mathcal{E}_{M^{*}}^{0}(G)$. The required inequality for $G$ then follows from what we have just done, and the formula (5.23) for $S_{M}^{G}\left(\sigma_{T}, f^{S}\right)$. This completes the proof of Lemma 5.3.

Returning to the proof of Lemma 5.2, we write the left hand side of (5.18) as

$$
\lim _{T \underset{P, r}{\longrightarrow}} \sum_{\left\{\left(L_{1}^{\prime}, M_{1}\right)\right\}} \sum_{u} \widehat{S}_{\tilde{M}^{\prime}}^{\tilde{G}^{\prime}}\left(\sigma_{T}^{\prime},\left(f^{\prime}\right)^{S^{\prime}}\right), \quad S^{\prime}=(u T)_{1}^{\prime},
$$

with $\left\{\left(L_{1}^{\prime}, M_{1}\right)\right\}$ and $u$ summed as in (5.20) and (5.21) respectively. We shall apply Lemma 5.3 to each of the summands. We can choose the representative $\left(L_{1}^{\prime}, M_{1}\right)$ of a given class so that both $M^{\prime}$ and $L_{1}^{\prime}$ are standard with respect to a fixed minimal parabolic subgroup $P_{0}^{\prime} \in \mathcal{P}\left(M_{0}^{\prime}\right)$ for $G^{\prime}$, both $M^{*}$ and $M_{1}^{*}$ are standard with respect to a fixed minimal parabolic subgroup $P_{0}^{*} \in \mathcal{P}\left(M_{0}^{*}\right)$ for $G^{*}$ and so that there is a fixed admissible embedding $\mathfrak{a}_{M_{0}^{\prime}} \hookrightarrow \mathfrak{a}_{M_{0}^{*}}$ such that the intersection of the closure of $\mathfrak{a}_{P_{0}^{*}}^{+}$with the chamber $\mathfrak{a}_{P_{0}^{\prime}}^{+}$contains an open cone $c_{0}^{\prime}=\left(c_{0}^{\prime}\right)^{+}$ in $\mathfrak{a}_{M_{0}^{\prime}}$. As an elliptic endoscopic datum for $M, M^{\prime}$ comes with an admissible isomorphism $\mathfrak{a}_{M^{\prime}} \stackrel{\sim}{\longrightarrow} \mathfrak{a}_{M}=\mathfrak{a}_{M^{\prime}}$. The chamber $\mathfrak{a}_{P}^{r}$ is an open cone in the chamber $\mathfrak{a}_{P^{\prime}}^{+}$in $\mathfrak{a}_{M^{\prime}}$ attached to a unique group $P^{\prime} \in \mathcal{P}\left(M^{\prime}\right)$. We claim that if $T$ lies in $\mathfrak{a}_{P}^{r}$, and $S^{\prime}=(u T)_{1}^{\prime}$ as in $(5.24)$, then $T$ is $\left(c_{0}^{\prime}, S^{\prime}\right)$-dominant, and the distance function

$$
d_{c_{0}^{\prime}}\left(T, S^{\prime}\right)=\inf _{w^{\prime} \in W\left(M_{0}^{\prime}\right)}\left\|T-w^{\prime} S^{\prime}\right\|
$$

is bounded below by a constant multiple of $\|T\|$. The first assertion follows from standard properties of convex hulls, and the fact that the dual chamber ${ }^{+} c_{0}^{\prime}$ of $c_{0}^{\prime}$ in $\mathfrak{a}_{M_{0}^{\prime}}$ contains the dual ${ }^{+} \mathfrak{a}_{P_{0}^{\prime}}$ of $\mathfrak{a}_{P_{0}^{\prime}}^{+}$. The second assertion follows from the fact that the closures of $\mathfrak{a}_{P}^{r}$ and $w^{\prime} u \mathfrak{a}_{P}^{r}$ in $\mathfrak{a}_{M_{0}^{\prime}}$ intersect only at the origin if $u$ belongs 
to $U\left(M, L_{1}\right)$, and the fact that

$$
\left\|w^{\prime} S^{\prime}\right\|=\left\|w^{\prime}(u T)_{1}^{\prime}\right\|=\left\|(u T)_{1}^{\prime}\right\|<\delta_{1}\|T\|,
$$

for some fixed $\delta_{1}<1$, if $u$ lies in the complement of $U\left(M, L_{1}\right)$.

We can now apply Lemma 5.3, with $\widetilde{G}^{\prime}$ in place of $G$, to each summand in (5.24). We have just shown that the conditions of this lemma apply to any point $T$ in the subset $\mathfrak{a}_{P}^{r}$ of $c_{0}^{\prime}$, and that

$$
d_{c_{0}^{\prime}}\left(T, S^{\prime}\right) \geq \varepsilon\|T\|
$$

for some $\varepsilon>0$. We conclude that the limit (5.24) vanishes uniformly for $\sigma^{\prime} \in \Gamma^{\prime}$. The original limit (5.18) therefore also vanishes uniformly for $\sigma^{\prime} \in \Gamma^{\prime}$, as required. This completes the proof of Lemma 5.2.

Lemma 5.2 allows us to replace (5.17) by an expression whose limit we can handle inductively. The process can be regarded as a stabilization of the left hand side of (5.1), for any Schwartz function $f \in \mathcal{C}(G, \zeta)$.

In fact, we have done enough to stabilize the entire limit formula (5.1), so long as we again restrict $f$ to the Hecke algebra $\mathcal{H}(G, \zeta)$. We state the final result formally as a corollary of Proposition 5.1, though it is really a culmination of all the discussions of this section.

Corollary 5.4. (a) If $G$ is arbitrary, then

$$
\lim _{T \underset{P, r}{\longrightarrow}} I_{M}^{\mathcal{E}}\left(\sigma_{T}^{\prime}, f_{T}\right)=I_{M}^{P}\left(\sigma^{\prime}, f\right), \quad f \in \mathcal{H}(G, \zeta),
$$

uniformly for $\sigma^{\prime} \in \Gamma^{\prime}$. In particular, this limit equals the limit on the left hand side of (5.13).

(b) If $G$ is quasisplit, the limit

$$
\lim _{T \underset{P, r}{\longrightarrow} \infty} S_{M}^{G}\left(M^{\prime}, \sigma_{T}^{\prime}, f_{T}\right), \quad f \in \mathcal{H}(G, \zeta),
$$

converges uniformly for $\sigma^{\prime} \in \Gamma^{\prime}$, and vanishes unless $\left(M^{\prime}, \sigma^{\prime}\right)=\left(M^{*}, \sigma^{*}\right)$, in which case it equals $\widehat{S}_{M^{*}}^{G^{*}, P^{*}}\left(\sigma^{*}, f^{*}\right)$. In particular, we have

$$
\lim _{T \underset{P, r}{\longrightarrow}} S_{M}^{G}\left(\sigma_{T}, f_{T}\right)=S_{M}^{G, P}(\sigma, f), \quad \sigma \in T_{G \text {-reg }}(\mathbb{R}),
$$

so this last limit is stable in $f \in \mathcal{H}(G, \zeta)$.

Proof. We assume inductively that for any $G^{\prime} \in \mathcal{E}_{M^{\prime}}^{0}(G)$,

$$
\lim _{T \underset{\tilde{P}^{\prime}, r}{\longrightarrow}} \widehat{S}_{\tilde{M}^{\prime}}^{\tilde{G}^{\prime}}\left(\sigma_{T}^{\prime},\left(f^{\prime}\right)_{T}\right)=\widehat{S}_{\tilde{M}^{\prime}}^{\tilde{G}^{\prime}, \tilde{P}^{\prime}}\left(\sigma^{\prime}, f^{\prime}\right),
$$

uniformly for $\sigma^{\prime} \in \Gamma^{\prime}$. Together with the assertion (5.18) of Lemma 5.2 and the fact that $\mathfrak{a}_{\tilde{P}^{\prime}}^{r}$ contains $\mathfrak{a}_{P}^{r}$, this implies that

$$
\begin{aligned}
& \lim _{T \underset{P, r}{\longrightarrow} \infty} \sum_{G^{\prime} \in \mathcal{E}_{M^{\prime}}^{0}(G)} \iota_{M^{\prime}}\left(G, G^{\prime}\right) \widehat{S}_{\tilde{M}^{\prime}}^{\tilde{G}^{\prime}}\left(\sigma_{T}^{\prime}, f_{T}^{\prime}\right) \\
& =\sum_{G^{\prime} \in \mathcal{E}_{M^{\prime}}^{0}(G)} \iota_{M^{\prime}}\left(G, G^{\prime}\right) \lim _{T \underset{P, r}{\infty}} \widehat{S}_{\tilde{M}^{\prime}}^{\tilde{G}^{\prime}}\left(\sigma_{T}^{\prime},\left(f^{\prime}\right)_{T}\right) \\
& =\sum_{G^{\prime} \in \mathcal{E}_{M^{\prime}}^{0}(G)} \iota_{M^{\prime}}\left(G, G^{\prime}\right) \widehat{S}_{\tilde{M}^{\prime}}^{\tilde{G}^{\prime}, \tilde{P}^{\prime}}\left(\sigma^{\prime}, f^{\prime}\right),
\end{aligned}
$$


uniformly for $\sigma^{\prime} \in \Gamma^{\prime}$. According to the stabilization (5.11) we have deduced as a consequence of Proposition 5.1, this last sum can be written in turn as

$$
I_{M}^{P}\left(\sigma^{\prime}, f\right)-\varepsilon\left(G, M^{\prime}\right) \widehat{S}_{M^{*}}^{G^{*}, P^{*}}\left(\sigma^{*}, f^{*}\right),
$$

for $\varepsilon\left(G, M^{\prime}\right)$ as in the proof of Lemma 4.1. Namely, $\varepsilon\left(G, M^{\prime}\right)=0$ unless $G$ is quasisplit and $\left(M^{\prime}, \sigma^{\prime}\right)$ equals $\left(M^{*}, \sigma^{*}\right)$, in which case $\varepsilon\left(G, M^{\prime}\right)=1$.

We have established a uniform limit formula for the sum (5.17). The same formula therefore holds for the difference (5.16) with which we began. Namely, the limit of (5.16) converges uniformly for $\sigma^{\prime} \in \Gamma^{\prime}$ to (5.27). The assertions of the corollary then follow directly from the definitions, as for example in the proof of Lemma 4.1. Notice that the induction argument based on (5.26) is resolved by the formula (5.25) (together with the running induction assumption that $S_{\tilde{M}^{\prime}}^{\tilde{G}^{\prime}}\left(\sigma_{T}^{\prime}, \cdot\right)$ is stable, which will be resolved finally in the coming section).

\section{§6. Proof of the theorem}

We are now ready to prove Theorem 1.1. We recall that $M^{\prime}$ is an elliptic endoscopic datum for $M$ with a maximal torus $T^{\prime} \subset M^{\prime}$ over $\mathbb{R}$, that $\Delta_{M}$ is a transfer factor for $M$ and $M^{\prime}$ with auxiliary datum $\left(\widetilde{M}^{\prime}, \widetilde{\xi}_{M}^{\prime}\right)$, and that $\sigma^{\prime}$ represents a strongly $G$-regular point in the corresponding torus $\widetilde{T}^{\prime}(\mathbb{R})$. The assertions of Theorem 1.1 can be formulated as the vanishing of certain functions of $\sigma^{\prime}$.

We define

$$
\varepsilon_{M}\left(\sigma^{\prime}, f\right)=I_{M}^{\mathcal{E}}\left(\sigma^{\prime}, f\right)-I_{M}\left(\sigma^{\prime}, f\right), \quad f \in \mathcal{C}(G, \zeta) .
$$

Part (a) of Theorem 1.1 asserts that this function vanishes. If $G$ is quasisplit, we also set

$$
\varepsilon^{M^{\prime}}\left(\sigma^{\prime}, f\right)=S_{M}^{G}\left(M^{\prime}, \sigma^{\prime}, f\right), \quad f \in \mathcal{C}(G, \zeta)
$$

In the further case that $M^{\prime}=M^{*}$, we assume implicitly that $f$ is unstable, in the sense that $f^{G}=0$. With this condition on $f$, part (b) of the theorem is the assertion that $\varepsilon^{M^{\prime}}\left(\sigma^{\prime}, f\right)$ vanishes. In general, it is clear that as $\Delta_{M}$ and $\sigma^{\prime}$ vary, $\varepsilon_{M}\left(\sigma^{\prime}, f\right)$ and $\varepsilon^{M^{\prime}}\left(\sigma^{\prime}, f\right)$ represent sections in $C^{\infty}\left(T_{G \text {-reg }}^{\prime}, M, \zeta\right)$. It suffices to fix $\Delta_{M}$, and study these objects as functions in $C^{\infty}\left(\widetilde{T}_{G \text {-reg }}^{\prime}, \zeta\right)$.

If $G$ is quasisplit, $\varepsilon_{M}\left(\sigma^{\prime}, f\right)$ vanishes by definition. We can therefore treat both cases of the theorem together by setting

$$
\varepsilon_{M}^{\prime}\left(\sigma^{\prime}, f\right)= \begin{cases}\varepsilon_{M}\left(\sigma^{\prime}, f\right), & \text { if } G \text { is not quasisplit } \\ \varepsilon^{M^{\prime}}\left(\sigma^{\prime}, f\right), & \text { if } G \text { is quasisplit. }\end{cases}
$$

Let us also set $\mathcal{C}^{\prime}(G, \zeta)$ equal to $\mathcal{C}(G, \zeta)$ unless $G$ is quasisplit and $M^{\prime}=M^{*}$, in which case we take $\mathcal{C}^{\prime}(G, \zeta)$ to be the closed subspace of unstable functions in $\mathcal{C}(G, \zeta)$. The assertion we have to establish is that $\varepsilon_{M}^{\prime}\left(\sigma^{\prime}, f\right)$ vanishes for any $\sigma^{\prime} \in \widetilde{T}_{G \text {-reg }}^{\prime}(\mathbb{R})$ and $f \in \mathcal{C}^{\prime}(G, \zeta)$.

We have been working up to this point with a partial induction assumption. We now take on the full assumption, based on the two integers $d_{\text {der }}$ and $r_{\text {der }}$ at the end of $\S 2$. We suppose from now on that the required assertion holds if $\left(G, M, M^{\prime}\right)$ is 
replaced by any triplet $\left(G_{1}, M_{1}, M_{1}^{\prime}\right)$ that satisfies one of the conditions

$$
\begin{gathered}
\operatorname{dim}\left(G_{1, \mathrm{der}}\right)<d_{\text {der }}, \\
\operatorname{dim}\left(G_{1, \mathrm{der}}\right)=d_{\text {der }}, \varepsilon\left(G_{1}\right)=1, \text { and } \varepsilon(G)=0,
\end{gathered}
$$

or

$$
\operatorname{dim}\left(G_{1, \mathrm{der}}\right)=d_{\mathrm{der}}, \text { and } \operatorname{dim}\left(A_{M_{1}} \cap G_{1, \mathrm{der}}\right)<r_{\mathrm{der}} .
$$

Conditions (1) and (2) are to accommodate an argument of increasing induction on $d_{\mathrm{der}}$, which requires that we treat the case of quasisplit $G$ first. (Notice that (2) includes the requirement that $G_{1}$ be quasisplit, and $G$ not be quasisplit.) Together, they include the initial assumption we took on in $\$ 1$ in order that the terms in the original definitions make sense. Condition (3) is designed for a supplementary argument of decreasing induction on $r_{\text {der }}$. It includes the assumption that $\varepsilon_{L}^{\prime}\left(\sigma^{\prime}, f\right)$ vanishes for any Levi subgroup $L \in \mathcal{L}(M)$ that properly contains $M$, and will be used repeatedly in what follows.

As we noted at the end of $\S 1$, the descent formulas (2.12)-(2.15) imply that $\varepsilon_{M}^{\prime}\left(\sigma^{\prime}, f\right)$ vanishes if $T^{\prime}$ is not elliptic in $M^{\prime}$. We therefore assume henceforth that $T^{\prime}$ is elliptic. Our concern now will be the finer analytic properties of $\varepsilon_{M}^{\prime}\left(\sigma^{\prime}, f\right)$, as a smooth function of $\sigma \in \widetilde{T}_{G \text {-reg }}^{\prime}(\mathbb{R})$. We shall study them by combining our general induction hypothesis with the results of $\S 2-5$.

Consider the differential equations of $\S 2$. In the case that $G$ is not quasisplit, we combine the two sets of equations (2.8) and (2.9) satisfied by $I_{M}\left(\sigma^{\prime}, f\right)$ and $I_{M}^{\mathcal{E}}\left(\sigma^{\prime}, f\right)$ respectively. Subtracting one equation from the other, we see that they may be written together as

$$
\varepsilon_{M}\left(\sigma^{\prime}, z f\right)=\sum_{L \in \mathcal{L}(M)} \partial_{M}^{L}\left(\sigma^{\prime}, z_{L}\right) \varepsilon_{L}\left(\sigma^{\prime}, f\right),
$$

for any element $z \in \mathcal{Z}(G, \zeta)$. If $L$ properly contains $M$, our induction condition (3) tells us that $\varepsilon_{L}\left(\sigma^{\prime}, f\right)$ vanishes. Since

$$
\partial_{M}^{M}\left(\sigma^{\prime}, z_{M}\right)=\partial\left(h_{T}(z)\right)^{\prime}
$$

we see that

$$
\varepsilon_{M}\left(\sigma^{\prime}, z f\right)=\partial\left(h_{T}(z)\right)^{\prime} \varepsilon_{M}\left(\sigma^{\prime}, f\right) .
$$

If $G$ is quasisplit, we apply the equations (2.10) or (2.11), according to whether $M^{\prime}=M^{*}$ or not. In case $M^{\prime}=M^{*}$, the same condition (3) tells us that $S_{L}^{G}(\sigma, f)$ vanishes for any Levi subgroup $L$ that properly contains $M$, any point $\sigma$ in $T_{G \text {-reg }}(\mathbb{R})$, and any $f \in \mathcal{C}(G, \zeta)$ with the required property that $f^{G}=0$. It follows from the two sets of equations that

$$
\varepsilon^{M^{\prime}}\left(\sigma^{\prime}, z f\right)=\partial\left(h_{T}(z)\right)^{\prime} \varepsilon^{M^{\prime}}\left(\sigma^{\prime}, f\right) .
$$

In the case that $M^{\prime}=M^{*}$, the function $z f$ is also unstable since

$$
(z f)^{G}=z^{G} f^{G}=0,
$$

so the notation $\varepsilon^{M^{\prime}}\left(\sigma^{\prime}, z f\right)$ here is consistent. We conclude that

$$
\partial\left(h_{T}(z)\right)^{\prime} \varepsilon_{M}^{\prime}\left(\sigma^{\prime}, f\right)=\varepsilon_{M}^{\prime}\left(\sigma^{\prime}, z f\right), \quad z \in \mathcal{Z}(G, \zeta),
$$

in all cases. 
Consider next the noncompact boundary conditions of $\S 4$. Suppose that $\beta^{\prime}$ is a real root of $T^{\prime}$. It follows from our induction hypothesis (3) and the definitions at the beginning of $\S 4$ that

$$
\begin{aligned}
\varepsilon_{M}\left(\sigma^{\prime}, f\right) & =I_{M}^{\mathcal{E}}\left(\sigma^{\prime}, f\right)-I_{M}\left(\sigma^{\prime}, f\right) \\
& =I_{M}^{\mathcal{E}, \beta}\left(\sigma^{\prime}, f\right)-I_{M}^{\beta}\left(\sigma^{\prime}, f\right),
\end{aligned}
$$

if $G$ is not quasisplit, and that

$$
\varepsilon^{M^{\prime}}\left(\sigma^{\prime}, f\right)=S_{M}^{G}\left(M^{\prime}, \sigma^{\prime}, f\right)=S_{M}^{G, \beta}\left(M^{\prime}, \sigma^{\prime}, f\right),
$$

if $G$ is quasisplit. The formulas (4.20)-(4.22) can therefore be stated uniformly as jump conditions for the function $\varepsilon_{M}^{\prime}\left(\sigma^{\prime}, f\right)$. To do so, we have of course to specialize $\sigma^{\prime}$ temporarily to a point in general position in the kernel $\left(\widetilde{T}^{\prime}\right)^{\beta^{\prime}}(\mathbb{R})$. The jump formulas then take the form

$$
j_{\beta^{\prime}}\left(D^{\prime} \varepsilon_{M}^{\prime}\left(\sigma^{\prime}, f\right)\right)=\lim _{\theta \rightarrow 0} \varepsilon_{\beta}(\theta) \sum_{M_{\beta}^{\prime} \in \mathcal{E}_{M^{\prime}}^{\beta}\left(M_{\beta}\right)} D_{\beta}^{\prime} \varepsilon_{M_{\beta}}^{\prime}\left(\sigma_{\beta}^{\prime}(\theta), f\right),
$$

for any invariant differential operator $D^{\prime}$ on $C^{\infty}\left(T^{\prime}, M, \zeta\right)$. The functions $\varepsilon_{M_{\beta}}^{\prime}\left(\sigma_{\beta}^{\prime}(\theta), f\right)$ on the right are attached to Levi subgroups $M_{\beta}$ that properly contain $M$. They vanish, again by our induction hypothesis (3). It follows that

$$
j_{\beta^{\prime}}\left(D^{\prime} \varepsilon_{M}^{\prime}\left(\sigma^{\prime}, f\right)\right)=0 \text {. }
$$

The compact boundary conditions of $\S 3$ depend on an $R_{I}^{\prime}$-chamber $c$ for $T^{\prime}$. For any such $c$, we form the smooth function

$$
\varepsilon_{M, c}^{\prime}\left(\sigma^{\prime}, f\right)=\delta_{c}^{\prime}\left(\sigma^{\prime}\right) \varepsilon_{M}^{\prime}\left(\sigma^{\prime}, f\right), \quad \sigma^{\prime} \in \widetilde{T}_{G-\mathrm{reg}}^{\prime}(\mathbb{R}),
$$

on $\widetilde{T}_{G \text {-reg }}^{\prime}(\mathbb{R})$, and the automorphism

$$
D^{\prime} \longrightarrow D_{c}^{\prime}=\delta_{c}^{\prime} \cdot D^{\prime} \circ\left(\delta_{c}^{\prime}\right)^{-1}
$$

of the linear space of invariant differential operators on $C^{\infty}\left(T^{\prime}, M, \zeta\right)$. The differential equations (6.2) can then be written

$$
\partial\left(h_{T}(z)\right)_{c}^{\prime} \varepsilon_{M, c}^{\prime}\left(\sigma^{\prime}, f\right)=\varepsilon_{M, c}^{\prime}\left(\sigma^{\prime}, z f\right) .
$$

The boundary conditions (6.3) we have already obtained can be written

$$
j_{\beta^{\prime}}\left(D_{c}^{\prime} \varepsilon_{M, c}^{\prime}\left(\sigma^{\prime}, f\right)\right)=0
$$

or if we prefer,

$$
j_{\beta^{\prime}}\left(D^{\prime} \varepsilon_{M, c}^{\prime}\left(\sigma^{\prime}, f\right)\right)=0,
$$

since $D^{\prime}$ is an arbitrary invariant differential operator on $C^{\infty}\left(T^{\prime}, M, \zeta\right)$.

Suppose that $\alpha^{\prime}$ is an imaginary root of $T^{\prime}$, and that $\sigma^{\prime}$ is specialized temporarily to a point in general position in the kernel $\left(\widetilde{T}^{\prime}\right)^{\alpha^{\prime}}(\mathbb{R})$. Since Proposition 3.2 applies to any of the functions from which $\varepsilon_{M, c}^{\prime}(\cdot, f)$ was constructed, we can use it to describe the jumps of $\varepsilon_{M, c}^{\prime}(\cdot, f)$ about $\alpha^{\prime}$. If $\alpha^{\prime}$ satisfies condition (i) of the proposition, we have

$$
j_{\alpha^{\prime}}\left(D_{c}^{\prime} \varepsilon_{M, c}^{\prime}\left(\sigma^{\prime}, f\right)\right)=\lim _{r \rightarrow 0} D_{c, \alpha}^{\prime} \varepsilon_{M, c_{\alpha}}^{\prime}\left(\sigma_{\alpha}^{\prime}(r), f\right),
$$

where $D_{c, \alpha}^{\prime}$ is the differential operator on $C^{\infty}\left(T_{\alpha}^{\prime}, M, \zeta\right)$ in (3.4). As a Cayley transform of $T^{\prime}$, the torus $T_{\alpha}^{\prime}$ in $M^{\prime}$ is not elliptic. Therefore $\varepsilon_{M, c_{\alpha}}^{\prime}\left(\sigma_{\alpha}^{\prime}(r), f\right)$ vanishes, by the descent formulas (2.12)-(2.14) and our induction hypothesis (1). 
The jump thus equals 0 in this case. If $\alpha^{\prime}$ satisfies condition (ii) of the proposition, the jump automatically vanishes. On the other hand, if $\alpha^{\prime}$ does not satisfy condition (ii), the root $w^{\prime} \alpha^{\prime}$ satisfies condition (i), for some element $w^{\prime}$ in the real Weyl group $W_{\mathbb{R}}\left(M^{\prime}, T^{\prime}\right)=W_{I}^{\prime}$. Since the transform of $\varepsilon_{M, c}^{\prime}(\cdot, f)$ by any element in $W_{I}^{\prime}$ equals the product of $\varepsilon_{M, c}^{\prime}(\cdot, f)$ with a smooth function (3.7), the jump vanishes in this case as well. We conclude that

$$
j_{\alpha^{\prime}}\left(D_{c}^{\prime} \varepsilon_{M, c}^{\prime}\left(\sigma^{\prime}, f\right)\right)=0
$$

in all cases.

Lemma 6.1. The function

$$
\varepsilon_{M, c}^{\prime}(f): \sigma^{\prime} \longrightarrow \varepsilon_{M, c}^{\prime}\left(\sigma^{\prime}, f\right), \quad \sigma^{\prime} \in \widetilde{T}_{G-\mathrm{reg}}^{\prime}(\mathbb{R}),
$$

extends to a $\left(\widetilde{\zeta}^{\prime}\right)^{-1}$-equivariant Schwartz function on $\widetilde{T}^{\prime}(\mathbb{R})$, and the correspondence $f \rightarrow \varepsilon_{M, c}^{\prime}(f)$ is a continuous linear mapping from $\mathcal{C}^{\prime}(G, \zeta)$ to $\mathcal{C}\left(\widetilde{T}^{\prime}, \widetilde{\zeta}^{\prime}\right)$.

Proof. We have fixed a Euclidean norm $\|\cdot\|$ on $\mathfrak{a}_{M}$. Its restriction to the orthogonal complement of $\mathfrak{a}_{Z}$ in $\mathfrak{a}_{M}$ can be regarded as a $\mathfrak{a}_{Z}$-invariant function $\|\cdot\|_{Z}$ on $\mathfrak{a}_{M}$, which in turn becomes an $\mathfrak{a}_{\tilde{Z}^{\prime}}$-invariant function on $\mathfrak{a}_{\tilde{M}^{\prime}}$. We then obtain a function

$$
\left\|\sigma^{\prime}\right\|=\left\|H_{\tilde{M}^{\prime}}\left(\sigma^{\prime}\right)\right\|_{Z}, \quad \sigma^{\prime} \in \widetilde{T}^{\prime}(\mathbb{R}),
$$

on $\widetilde{T}^{\prime}(\mathbb{R}) / \widetilde{Z}^{\prime}(\mathbb{R})$. The Schwartz space $\mathcal{C}\left(\widetilde{T}^{\prime}, \widetilde{\zeta}^{\prime}\right)$ is the space of smooth $\left(\widetilde{\zeta}^{\prime}\right)^{-1}$ equivariant functions $\phi^{\prime}$ on $\widetilde{T}^{\prime}(\mathbb{R})$ such that for every $n \geq 0$ and every invariant differential operator $D^{\prime}$ on $\widetilde{T}^{\prime}(\mathbb{R})$, the seminorm

$$
\sup _{\sigma^{\prime} \in \tilde{T}^{\prime}(\mathbb{R})}\left(\left\|D^{\prime} \phi^{\prime}\left(\sigma^{\prime}\right)\right\|\left(1+\left\|\sigma^{\prime}\right\|\right)^{n}\right)
$$

is finite.

We have also to introduce a function $\delta^{\prime}\left(\sigma^{\prime}\right)$ on $\widetilde{T}^{\prime}(\mathbb{R}) / \widetilde{Z}^{\prime}(\mathbb{R})$ that measures the distance to the $G$-singular set. The $G$-singular set in $\widetilde{T}^{\prime}(\mathbb{R})$ is a union

$$
\widetilde{\mathcal{S}}^{\prime}=\bigcup_{\alpha^{\prime}}\left\{\sigma_{1}^{\prime} \in \widetilde{T}^{\prime}(\mathbb{R}): \sigma_{1}^{\prime} \in \widetilde{T}^{\prime}(\mathbb{R}), \alpha^{\prime}\left(\sigma_{1}^{\prime}\right)=1\right\}
$$

of kernels, taken over all roots $\alpha^{\prime}$ of $T^{\prime}$ in the general sense defined at the beginning of $\S 3$. The complement of $\widetilde{\mathcal{S}}^{\prime}$ in $\widetilde{T}^{\prime}(\mathbb{R})$ is the set of $G$-regular elements in $\widetilde{T}^{\prime}(\mathbb{R})$, an open set that contains the set $\widetilde{T}_{G \text {-reg }}^{\prime}(\mathbb{R})$ of strongly $G$-regular elements. We extend $\|\cdot\|_{Z}$ to a Euclidean norm on $\widetilde{\mathfrak{t}}^{\prime}(\mathbb{R}) / \widetilde{\mathfrak{z}}^{\prime}(\mathbb{R})$ whose inverse image under any $M$-admissible isomorphism from $T$ to $T^{\prime}$ is a $W_{\mathbb{R}}(G, T)$-invariant norm on $\mathfrak{t}(\mathbb{R}) / \mathfrak{z}(\mathbb{R})$. Let $U^{\prime}$ be a small fixed neighbourhood of 1 in $\widetilde{T}^{\prime}(\mathbb{R}) / \widetilde{Z}^{\prime}(\mathbb{R})$. We set $\delta^{\prime}\left(\sigma^{\prime}\right)=1$ unless $\sigma^{\prime} U^{\prime}$ intersects $\widetilde{S}^{\prime}$, in which case we set

$$
\delta^{\prime}\left(\sigma^{\prime}\right)=\inf _{\left\{h^{\prime} \in U^{\prime}: \sigma^{\prime} h^{\prime} \in \tilde{\mathcal{S}}^{\prime}\right\}}\left(\left\|\log h^{\prime}\right\|_{Z}\right) .
$$

The function $\varepsilon_{M}^{\prime}\left(\sigma^{\prime}, f\right)$ of $\sigma^{\prime} \in \widetilde{T}_{G \text {-reg }}^{\prime}(\mathbb{R})$ extends to a smooth function on the larger open set $\widetilde{T}^{\prime}(\mathbb{R})-\widetilde{\mathcal{S}}^{\prime}$ of $G$-regular elements. This follows from a natural variant of Lemma 3.1 that applies to points $\sigma_{1}^{\prime}$ in $\widetilde{T}^{\prime}(\mathbb{R})-\widetilde{\mathcal{S}}^{\prime}$, since the property A4, (2.3)] on which the lemma relies holds for any element $\gamma_{1} \in M(\mathbb{R})$ whose connected centralizer in $G$ is contained in $M$. We claim that for every $n$, there is a continuous seminorm $\|\cdot\|_{n}$ on $\mathcal{C}(G, \zeta)$ such that

$$
\left|\varepsilon_{M}^{\prime}\left(\sigma^{\prime}, f\right)\right| \leq\|f\|_{n} \delta^{\prime}\left(\sigma^{\prime}\right)^{-1}\left(1+\left\|\sigma^{\prime}\right\|\right)^{-n},
$$


for every $\sigma^{\prime}$ in $\widetilde{T}^{\prime}(\mathbb{R})-\widetilde{\mathcal{S}}^{\prime}$ and $f \in \mathcal{C}^{\prime}(G, \zeta)$. The first step is to show that a similar estimate holds with $I_{M}\left(\sigma^{\prime}, f\right)$ in place of $\varepsilon_{M}^{\prime}\left(\sigma^{\prime}, f\right)$. The estimate in this case follows from the definition (1.4), the definition [A14, §1] of the invariant distribution $I_{M}(\gamma, f)$, and the original estimate [A2, Corollary 7.4] for its noninvariant analogue $J_{M}(\gamma, f)$. Similar estimates for $I_{M}^{\mathcal{E}}\left(\sigma^{\prime}, f\right)$ and $S_{M}^{G}\left(M^{\prime}, \sigma^{\prime}, f\right)$ follow inductively from (1.8)-(1.10), and the fact that $f \rightarrow f^{\prime}$ is a continuous linear mapping from $\mathcal{C}(G, \zeta)$ to $\mathcal{S}\left(\widetilde{G}^{\prime}, \widetilde{\zeta}^{\prime}\right)$. The required estimate (6.5) then follows from the definition of $\varepsilon_{M}^{\prime}\left(\sigma^{\prime}, f\right)$.

We shall now apply an important and well known technique of Harish-Chandra, by which we can use the differential equations (6.2) to extend the estimate (6.5) to derivatives. The basic idea was introduced in [H1, Lemma 48], and is quite familiar from other contexts $\mathrm{A} 2, \mathrm{~L} 2$ and $[\mathrm{AC}$ as well. We shall sketch the technique as it applies here, to see that it yields the kind of estimates we want at infinity in $\widetilde{T}^{\prime}(\mathbb{R})$.

Suppose that $D^{\prime}$ is an invariant differential operator on $C^{\infty}\left(T^{\prime}, M, \zeta\right)$. According to [H1, §25], there is an identity

$$
\sum_{j=1}^{r}\left(\partial\left(h_{T}\left(z_{i}\right)\right)^{\prime}\right)^{*} E_{j, \varepsilon}=\left(D^{\prime}\right)^{*} \delta+\beta_{\varepsilon}
$$

of distributions on the orthogonal complement $\widetilde{\mathfrak{t}}^{\prime}(\mathbb{R})_{Z}$ of $\widetilde{\mathfrak{z}}^{\prime}(\mathbb{R})$ in $\widetilde{\mathfrak{t}}^{\prime}(\mathbb{R})$. The notation is essentially that of [H1, p. 498] and [A2, p. 252], adapted to the context at hand. In particular, $\delta$ is the Dirac distribution at $0, \varepsilon$ is any positive number with $\varepsilon \leq \frac{1}{3}$, and $\left\{z_{j}: 1 \leq j \leq r\right\}$ are elements in $\mathcal{Z}(G, \zeta)$, while $\beta_{\varepsilon}(H)$ and

$$
E_{j, \varepsilon}(H)=\Psi_{\varepsilon}(H) E_{j}(H), \quad 1 \leq j \leq r,
$$

are functions supported on the ball of radius $3 \varepsilon$. We are regarding invariant differential operators on $\widetilde{T}^{\prime}(\mathbb{R})$ also as differential operators of constant coefficients on the Lie algebra $\widetilde{\mathfrak{t}}^{\prime}(\mathbb{R})$, and we are writing $X^{*}$ for the real adjoint of any such operator $X$. If $\sigma^{\prime}$ is any given point in $\widetilde{T}_{G-\text { reg }}^{\prime}(\mathbb{R})$, we set

$$
\varepsilon=\frac{1}{4} \delta^{\prime}\left(\sigma^{\prime}\right)
$$

We then evaluate the distributions on each side of the equation at the function

$$
H \longrightarrow \varepsilon_{M}^{\prime}\left(\sigma^{\prime} \exp H, f\right) \text {. }
$$

This gives a formula for $D^{\prime} \varepsilon_{M}^{\prime}\left(\sigma^{\prime}, f\right)$ as a difference of integrals

$$
\int \sum_{j=1}^{r}\left(\partial\left(h_{T}\left(z_{i}\right)\right)^{\prime} \varepsilon_{M}^{\prime}\left(\sigma^{\prime} \exp H, f\right)\right) E_{j, \varepsilon}(H) d H
$$

and

$$
\int \varepsilon_{M}^{\prime}\left(\sigma^{\prime} \exp H, f\right) \beta_{\varepsilon}(H) d H
$$

over $\widetilde{\mathfrak{t}}^{\prime}(\mathbb{R})_{Z}$. The function $E_{j, \varepsilon}$ is bounded independently of $\varepsilon$, while $\beta_{\varepsilon}(H)$ is bounded by a constant multiple of a power of $\varepsilon^{-1}=4 \delta^{\prime}\left(\sigma^{\prime}\right)^{-1}$. Since

$$
\partial\left(h_{T}\left(z_{i}\right)\right)^{\prime} \varepsilon_{M}^{\prime}\left(\sigma^{\prime} \exp H, f\right)=\varepsilon_{M}^{\prime}\left(\sigma^{\prime} \exp H, z_{i} f\right)
$$

we can apply the estimate (6.5) to each of the two integrals. We conclude that there is a nonnegative integer $q^{\prime}$, and a continuous seminorm $\|f\|_{D^{\prime}, n}$ for any positive 
integer $n$, such that

$$
\left|D^{\prime} \varepsilon_{M}^{\prime}\left(\sigma^{\prime}, f\right)\right| \leq\|f\|_{D^{\prime}, n} \delta^{\prime}\left(\sigma^{\prime}\right)^{-q^{\prime}}\left(1+\left\|\sigma^{\prime}\right\|\right)^{-n},
$$

for any $\sigma^{\prime}$ in $\widetilde{T}^{\prime}(\mathbb{R})-\widetilde{\mathcal{S}}^{\prime}$ and $f \in \mathcal{C}^{\prime}(G, \zeta)$.

The exponent $q^{\prime}=q^{\prime}\left(D^{\prime}\right)$ in (6.6) depends a priori on $D^{\prime}$. However, we can remove this dependence by selecting a set of generators $\left\{D_{1}^{\prime}, \ldots, D_{d}^{\prime}\right\}$ for the space of invariant differential operators on $C^{\infty}\left(T^{\prime}, M^{\prime}, \zeta\right)$ as a module over (the image of) $\mathcal{Z}(G, \zeta)$. Any invariant differential operator $D^{\prime}$ can then be written in the form

$$
D^{\prime}=D_{1}^{\prime} \partial\left(h_{T}\left(z_{1}\right)\right)^{\prime}+\cdots+D_{d}^{\prime} \partial\left(h_{T}\left(z_{d}\right)\right)^{\prime},
$$

for elements $z_{1}, \ldots, z_{d}$ in $\mathcal{Z}(G, \zeta)$. It follows from (6.2) that

$$
D^{\prime} \varepsilon_{M}^{\prime}\left(\sigma^{\prime}, f\right)=\sum_{i=1}^{d} D_{i}^{\prime} \varepsilon_{M}^{\prime}\left(\sigma^{\prime}, z_{i} f\right) .
$$

The estimate (6.6) then holds for any $D^{\prime}$, if we take

$$
q^{\prime}=\max _{1 \leq i \leq d} q^{\prime}\left(D_{i}^{\prime}\right) .
$$

A separate technique of Harish-Chandra [H1, Lemma 49] establishes that one can in fact take $q^{\prime}=0$. The technique is summarized in the following lemma, whose elementary proof we leave to the reader. (See [L2, pp. 21-22], [AC, p. 169].)

Lemma 6.2. Suppose that $\lambda_{1}, \ldots, \lambda_{k}$ are linear forms on $\mathbb{R}^{d}$, and that $\phi$ is a smooth function on the set

$$
B_{\mathrm{reg}}=\left\{\xi \in \mathbb{R}^{d}:\|\xi\| \leq 1, \prod_{i=1}^{k} \lambda_{i}(\xi) \neq 0\right\} .
$$

Assume that there is a nonnegative integer $q$ with the property that for any invariant differential operator $D$ on $\mathbb{R}^{d}$,

$$
|D \phi(\xi)| \leq c_{D}\left|\prod_{i=1}^{k} \lambda_{i}(\xi)\right|^{-q}, \quad \xi \in B_{\mathrm{reg}},
$$

for a constant $c_{D}$ that depends on $D$. Then for any $D$, we can choose a constant of the form

$$
c_{D}^{*}=c_{0}\left(\sum_{\alpha} c_{D_{\alpha}}\right)
$$

where $\left\{D_{\alpha}\right\}$ is a finite set of invariant differential operators that depends only on $D$, and $c_{0}$ is independent of $D$ and $\phi$, such that

$$
|D \phi(\xi)| \leq c_{D}^{*}, \quad \xi \in B_{\text {reg }} .
$$

It is clear how to combine Lemma 6.2 with the estimate (6.6). Together, they imply that for any $D^{\prime}$ and $n$, there is a continuous seminorm $\|\cdot\|_{D^{\prime}, n}$ on $\mathcal{C}^{\prime}(G, \zeta)$ such that

$$
\left|D^{\prime} \varepsilon_{M}^{\prime}\left(\sigma^{\prime}, f\right)\right| \leq\|f\|_{D^{\prime}, n}\left(1+\left\|\sigma^{\prime}\right\|\right)^{-n},
$$

for any $\sigma^{\prime}$ in $\widetilde{T}^{\prime}(\mathbb{R})-\widetilde{\mathcal{S}}^{\prime}$ and $f \in \mathcal{C}^{\prime}(G, \zeta)$.

Suppose that $\Omega$ is a connected component in the complement of $\widetilde{\mathcal{S}}^{\prime}$ in $\widetilde{T}^{\prime}(\mathbb{R})$. The estimate (6.7) implies that $\varepsilon_{M}^{\prime}\left(\sigma^{\prime}, f\right)$ extends to a Schwartz function on the closure of $\Omega$, in the sense that there is a $\left(\widetilde{\zeta}^{\prime}\right)^{-1}$-equivariant Schwartz function on $\widetilde{T}^{\prime}(\mathbb{R})$ 
whose restriction to $\Omega$ equals $\varepsilon_{M}^{\prime}\left(\sigma^{\prime}, f\right)$. One observes without difficulty that the factor $\delta_{c}^{\prime}\left(\sigma^{\prime}\right)$ extends to a smooth function on the closure of $\Omega$, whose derivatives are tempered. It follows that the product $\varepsilon_{M, c}^{\prime}\left(\sigma^{\prime}, f\right)$ also extends to a Schwartz function on the closure of $\Omega$. As $\Omega$ varies, we thus have a family

$$
\left\{\varepsilon_{M, c}^{\prime}\left(\sigma^{\prime}, f\right): \sigma^{\prime} \in \Omega\right\}
$$

of Schwartz functions. The jump conditions $(6.3)_{c}$ and $(6.4)_{c}$ imply that these functions have compatible normal derivatives across common hypersurfaces. We conclude that $\varepsilon_{M, c}^{\prime}\left(\sigma^{\prime}, f\right)$ extends to a $\left(\widetilde{\zeta}^{\prime}\right)^{-1}$-equivariant Schwartz function $\varepsilon_{M, c}^{\prime}(f)$ on $\widetilde{T}^{\prime}(\mathbb{R})$. The estimate $(6.7)$ then tells us that the mapping $f \rightarrow \varepsilon_{M, c}^{\prime}(f)$ is continuous. This completes the proof of Lemma 6.1.

The next step is to take the Fourier transform of $\varepsilon_{M, c}^{\prime}(f)$, regarded now as a $\left(\widetilde{\zeta}^{\prime}\right)^{-1}$-equivariant Schwartz function on $\widetilde{T}^{\prime}(\mathbb{R})$. Since the function is invariant under the Weyl group $W\left(\widetilde{M}^{\prime}, \widetilde{T}^{\prime}\right)$, we may as well make use of the family of $W\left(\widetilde{M}^{\prime}, \widetilde{T}^{\prime}\right)$ invariant eigenfunctions provided by the set $\Phi_{\text {temp,cusp }}\left(\tilde{M}^{\prime}, \widetilde{\zeta}^{\prime}\right)$ of tempered, cuspidal, $\widetilde{\zeta}^{\prime}$-equivariant Langlands parameters for $\widetilde{M}^{\prime}$. Any $\phi^{\prime}$ in this family has a normalized stable character

$$
\eta_{M}^{\prime}\left(\phi^{\prime}, \sigma^{\prime}\right)=\sum_{\pi^{\prime} \in \Pi_{\phi^{\prime}}} \theta_{M}^{\prime}\left(\pi^{\prime}, \sigma^{\prime}\right)
$$

where

$$
\theta_{M}^{\prime}\left(\pi^{\prime}, \sigma^{\prime}\right)=\left|D^{\tilde{M}^{\prime}}\left(\sigma^{\prime}\right)\right|^{\frac{1}{2}} \Theta_{M}^{\prime}\left(\pi^{\prime}, \sigma^{\prime}\right), \quad \sigma^{\prime} \in \widetilde{T}_{G-\mathrm{reg}}^{\prime}(\mathbb{R}),
$$

is the normalized character of the representation $\pi^{\prime}$. Set

$$
\varepsilon_{M}^{\prime}\left(\phi^{\prime}, f\right)=\left|W\left(M^{\prime}, T^{\prime}\right)\right|^{-1} \int_{\tilde{T}^{\prime}(\mathbb{R}) / \tilde{Z^{\prime}}(\mathbb{R})} \eta_{M}^{\prime}\left(\phi^{\prime}, \sigma^{\prime}\right) \varepsilon_{M}^{\prime}\left(\sigma^{\prime}, f\right) d \sigma^{\prime} .
$$

We can also write

$$
\varepsilon_{M}^{\prime}\left(\phi^{\prime}, f\right)=\left|W\left(M^{\prime}, T^{\prime}\right)\right|^{-1} \int_{\tilde{T}^{\prime}(\mathbb{R}) / \tilde{Z^{\prime}}(\mathbb{R})} \eta_{M,-c}^{\prime}\left(\phi^{\prime}, \sigma^{\prime}\right) \varepsilon_{M, c}^{\prime}\left(\sigma^{\prime}, f\right) d \sigma^{\prime},
$$

since $\delta_{c}^{\prime}\left(\sigma^{\prime}\right)$ is a complex number of absolute value 1 whose complex conjugate equals $\delta_{-c}^{\prime}\left(\sigma^{\prime}\right)$. The function

$$
\sigma^{\prime} \longrightarrow \eta_{M,-c}^{\prime}\left(\phi^{\prime}, \sigma^{\prime}\right)
$$

on $\widetilde{T}_{\text {reg }}^{\prime}(\mathbb{R})$ extends to a smooth function on $\widetilde{T}^{\prime}(\mathbb{R})$. Its explicit formula as a linear combination of characters on $\widetilde{T}^{\prime}(\mathbb{R})[S 1$, coupled with standard abelian Fourier analysis, yields an inversion formula

$$
\varepsilon_{M, c}^{\prime}\left(\sigma^{\prime}, f\right)=\int_{\Phi_{\text {temp }, \mathrm{cusp}}\left(\tilde{M}^{\prime}, \tilde{\zeta}^{\prime}\right)} \eta_{M, c}^{\prime}\left(\sigma^{\prime}, \phi^{\prime}\right) \varepsilon_{M}^{\prime}\left(\phi^{\prime}, f\right) d \phi^{\prime},
$$

where

$$
\eta_{M}^{\prime}\left(\sigma^{\prime}, \phi^{\prime}\right)=\overline{\eta_{M}^{\prime}\left(\phi^{\prime}, \sigma^{\prime}\right)}
$$

is the function introduced in $\S 5$. Multiplying each side by $\delta_{-c}^{\prime}\left(\sigma^{\prime}\right)$, we see that

$$
\varepsilon_{M}^{\prime}\left(\sigma^{\prime}, f\right)=\int_{\Phi_{\text {temp }, \text { cusp }}\left(\tilde{M}^{\prime}, \tilde{\zeta}^{\prime}\right)} \eta_{M}^{\prime}\left(\sigma^{\prime}, \phi^{\prime}\right) \varepsilon_{M}^{\prime}\left(\phi^{\prime}, f\right) d \phi^{\prime} .
$$

It is therefore enough to show that $\varepsilon_{M}^{\prime}\left(\phi^{\prime}, f\right)$ vanishes for any parameter $\phi^{\prime}$ in $\Phi_{\text {temp,cusp }}\left(\tilde{M}^{\prime}, \widetilde{\zeta}^{\prime}\right)$. 
The smooth function $\eta_{M,-c}^{\prime}\left(\phi^{\prime}, \sigma^{\prime}\right)$ on $\widetilde{T}^{\prime}(\mathbb{R})$ is an eigenfunction of the space of invariant differential operators on $\widetilde{T}^{\prime}(\mathbb{R})$. It follows from $(6.2)_{c}$, the fact that $\varepsilon_{M, c}^{\prime}\left(\sigma^{\prime}, f\right)$ is a smooth function on $\widetilde{T}^{\prime}(\mathbb{R})$, and the second formula above for $\varepsilon_{M}^{\prime}\left(\phi^{\prime}, f\right)$, that the linear form

$$
f \longrightarrow \varepsilon_{M}^{\prime}\left(\phi^{\prime}, f\right), \quad f \in \mathcal{C}^{\prime}(G, \zeta)
$$

is an invariant tempered eigendistribution of $\mathcal{Z}(G, \zeta)$. In other words,

$$
\varepsilon_{M}^{\prime}\left(\phi^{\prime}, z f\right)=\chi_{\phi}^{G}(z) \varepsilon_{M}^{\prime}\left(\phi^{\prime}, f\right), \quad z \in \mathcal{Z}(G, \zeta),
$$

for a character $\chi_{\phi}^{G}$ on the algebra $\mathcal{Z}(G, \zeta)$. To be precise,

$$
\chi_{\phi}^{G}(z)=\chi_{\phi}\left(z_{M}\right), \quad z \in \mathcal{Z}(G, \zeta),
$$

is induced from the infinitesimal character $\chi_{\phi}$ of the image $\phi$ of $\phi^{\prime}$ in $\Phi_{\text {temp }}(M, \zeta)$. We note for future reference that the imaginary part of $\chi_{\phi}$ can be represented by a linear form $\nu_{\phi}$ in $i \mathfrak{a}_{M}^{*}$. This is a consequence of the fact that $\phi^{\prime}$ is cuspidal.

The distribution $\varepsilon_{M}^{\prime}\left(\phi^{\prime}, f\right)$ is supported on characters, in the sense that it depends only on the image $f_{G}$ of $f$ in $\mathcal{I}(G, \zeta)$. This follows from the definitions and the corresponding property A7] of the distribution $I_{M}(\gamma, f)$. (Using the main theorem of $[\mathrm{A} 1$, one can in fact show that any invariant tempered distribution on $G(\mathbb{R})$ is supported on characters.) We claim that $\varepsilon_{M}^{\prime}\left(\phi^{\prime}, f\right)$ actually depends only on the image $f_{M}$ of $f_{G}$ in $\mathcal{I}(M, \zeta)$. To see this, we recall that there are free actions $\phi^{\prime} \rightarrow \phi_{\lambda}^{\prime}$ and $\phi \rightarrow \phi_{\lambda}$ of the vector space $i \mathfrak{a}_{M, Z}^{*}$ that commute with the mapping $\phi^{\prime} \rightarrow \phi$. The set $\Phi_{\text {temp,cusp }}\left(\tilde{M}^{\prime}, \widetilde{\zeta}^{\prime}\right)$ is in fact a discrete union of associated $i \mathfrak{a}_{M, Z^{*}}^{*}$-orbits. The imaginary part $\nu_{\phi_{\lambda}} \in i \mathfrak{a}_{M}^{*}$ satisfies the obvious identity

$$
\nu_{\phi_{\lambda}}=\nu_{\phi}+\lambda
$$

Consequently, if $\lambda$ is in general position, and $\pi$ is an irreducible tempered representation of $G(\mathbb{R})$ that is not parabolically induced from a tempered representation of $M(\mathbb{R})$, the infinitesimal character $\chi_{\pi}$ of $\pi$ is distinct from $\chi_{\phi_{\lambda}}$. Since $\varepsilon_{M}^{\prime}\left(\phi_{\lambda}^{\prime}, f\right)$ equals a (finite) linear combination of eigendistributions with infinitesimal character $\chi_{\phi_{\lambda}}$, the support of its invariant Fourier transform is disjoint from $\pi$. The claim follows from the fact that $\varepsilon_{M}^{\prime}\left(\phi_{\lambda}^{\prime}, f\right)$ is continuous in $\lambda$.

We need to say a word about the space of eigendistributions to which $\varepsilon_{M}^{\prime}\left(\phi^{\prime}, f\right)$ belongs. It is composed of induced distributions

$$
f_{G}\left(\rho^{G}\right)=f_{M}(\rho), \quad f \in \mathcal{C}(G, \zeta),
$$

obtained from invariant, $\zeta$-equivariant, tempered distributions $\rho$ on $M(\mathbb{R})$. Let $T_{\phi}^{+}$be the set of distributions on $M(\mathbb{R})$ that belong to $T_{\text {temp }}(M, \zeta)$, and whose infinitesimal character equals $\chi_{\phi}$. The corresponding induced family consists of eigendistributions on $G(\mathbb{R})$ with infinitesimal character $\chi_{\phi}^{G}$, but it might not span the space that contains $\varepsilon_{M}^{\prime}\left(\phi^{\prime}, f\right)$. Suppose that $\tau \in T_{\phi}^{+}$equals an induced virtual character $\tau_{1}^{M}$ attached to an elliptic element $\tau_{1} \in T_{\text {ell }}\left(M_{1}, \zeta\right)$, for some Levi subgroup $M_{1} \subset M$, and that $D$ is an invariant differential operator on the space $i \mathfrak{a}_{M_{1}}^{*} / i \mathfrak{a}_{M}^{*}$. The linear form

$$
h_{M}(\rho)=\lim _{\mu \rightarrow 0} D h_{M}\left(\tau_{1, \mu}^{M}\right), \quad h_{M} \in \mathcal{I}(M, \zeta),
$$


is a generalized eigendistribution for $\mathcal{Z}(M, \zeta)$ with infinitesimal character $\chi_{\phi}$. In other words,

$$
\left(z_{M}-\chi_{\phi}\left(z_{M}\right)\right)^{d} \rho=0, \quad z_{M} \in \mathcal{Z}(M, \zeta),
$$

for a positive integer $d$. It is known from examples that one can sometimes take $d=1$, even if $D$ is not constant. In other words, there can be invariant, tempered eigendistributions that do not lie in the span of $T_{\phi}^{+}$. This forces us to work with the (infinite dimensional) space $\mathcal{F}_{\phi}^{+}$spanned by distributions on $M(\mathbb{R})$ of the form (6.9). It follows easily from the trace Paley-Wiener theorem in A8 that if $\phi^{\prime}$ is in general position, the image of $\mathcal{F}_{\phi}^{+}$under the induction mapping $\rho \rightarrow \rho^{G}$ contains the (finite dimensional) space of invariant, $\zeta$-equivariant, tempered eigendistributions on $G(\mathbb{R})$ with infinitesimal character $\chi_{\phi}^{G}$. In particular, it contains the distribution $\varepsilon_{M}^{\prime}\left(\phi^{\prime}, f\right)$.

If $\lambda$ belongs to the space $i \mathfrak{a}_{M, Z}^{*}$, the twist $\rho_{\lambda}$ of any element in $\mathcal{F}_{\phi}^{+}$belongs to $\mathcal{F}_{\phi_{\lambda}}^{+}$. Let $\mathcal{R}_{\phi}^{+}$be a fixed basis of $\mathcal{F}_{\phi}^{+}$that contains $T_{\phi}^{+}$, and consists of distributions of the form (6.9). For any $\lambda$, the family

$$
\mathcal{R}_{\phi_{\lambda}}^{+}=\left\{\rho_{\lambda}: \rho \in \mathcal{R}_{\phi}^{+}\right\}
$$

is then a basis of $\mathcal{F}_{\phi_{\lambda}}^{+}$. It provides an expansion

$$
\varepsilon_{M}^{\prime}\left(\phi_{\lambda}^{\prime}, f\right)=\sum_{\rho \in \mathcal{R}_{\phi}^{+}} \varepsilon_{M}^{\prime}\left(\phi_{\lambda}^{\prime}, \rho_{\lambda}\right) f_{M}\left(\rho_{\lambda}\right)
$$

of the associated distribution, for complex numbers $\varepsilon_{M}^{\prime}\left(\phi_{\lambda}^{\prime}, \rho_{\lambda}\right)$ that vanish for almost all $\rho$.

We return to the problem of showing that $\varepsilon_{M}^{\prime}\left(\phi^{\prime}, f\right)$ vanishes for any given $\phi^{\prime}$. It will be convenient to take $\phi^{\prime}$ to be a parameter within a given $i \mathfrak{a}_{M, Z^{*}}^{*}$ orbit, such that the linear form $\nu_{\phi}$ is trivial on the kernel $\mathfrak{a}_{M}^{G}$ of the projection of $\mathfrak{a}_{M}$ onto $\mathfrak{a}_{G}$. In fact, we may as well fix a (noncanonical) isomorphism from $\mathfrak{a}_{M} / \mathfrak{a}_{Z}$ onto a complement $\mathfrak{a}_{M}^{Z}$ of $\mathfrak{a}_{Z}$ in $\mathfrak{a}_{M}$ that contains $\mathfrak{a}_{M}^{G}$. For example, we could take $\mathfrak{a}_{M}^{Z}$ to be the orthogonal complement of $\mathfrak{a}_{Z}$ relative to underlying (noncanonical) Euclidean inner product. We then take $\phi^{\prime}$ to be the parameter within the given $i \mathfrak{a}_{M, Z}^{*}$-orbit such that $\nu_{\phi}$ vanishes on $\mathfrak{a}_{M}^{Z}$. With this restriction, our task is to show that $\varepsilon_{M}^{\prime}\left(\phi_{\lambda}^{\prime}, f\right)$ vanishes for every $\lambda \in i \mathfrak{a}_{M, Z}^{*}$.

It is here that we will use the limit formula from A14, or rather its stabilization obtained in the last section. We are free to express $\varepsilon_{M}^{\prime}\left(\phi^{\prime}, f\right)$ as an iterated integral

for the function

$$
\varepsilon_{M}^{\prime}\left(\phi^{\prime}, f\right)=\int_{\mathfrak{a}_{M} / \mathfrak{a}_{Z}} \varepsilon_{M}\left(\phi^{\prime}, X, f\right) d X
$$

$$
\varepsilon_{M}^{\prime}\left(\phi^{\prime}, X, f\right)=\left|W\left(M^{\prime}, T^{\prime}\right)\right|^{-1} \int_{\tilde{T}^{\prime}(\mathbb{R})^{X}} \eta_{M}^{\prime}\left(\phi^{\prime}, \sigma^{\prime}\right) \varepsilon_{M}^{\prime}\left(\sigma^{\prime}, f\right) d \sigma^{\prime},
$$

defined in terms of an integral over the compact subset

$$
\widetilde{T}^{\prime}(\mathbb{R})^{X}=\left\{\sigma^{\prime} \in \widetilde{T}^{\prime}(\mathbb{R}) / \widetilde{Z}^{\prime}(\mathbb{R}): H_{\tilde{M}^{\prime}}\left(\sigma^{\prime}\right)=X\right\}
$$

of $\widetilde{T}^{\prime}(\mathbb{R}) / \widetilde{Z}^{\prime}(\mathbb{R})$. Suppose for the moment that $f$ belongs to the Hecke algebra $\mathcal{H}(G, \zeta)$, as in $\S 5$. We continue of course to assume that $f^{G}=0$ in the case that $G$ is quasisplit and $M^{\prime}=M^{*}$, which is to say that $f$ belongs to the subspace

$$
\mathcal{H}^{\prime}(G, \zeta)=\mathcal{H}(G, \zeta) \cap \mathcal{C}^{\prime}(G, \zeta)
$$


of $\mathcal{H}(G, \zeta)$. It then follows from (5.13), Corollary 5.4 and the definitions at the beginning of this section that

$$
\lim _{T, r} \varepsilon_{M}^{\prime}\left(\sigma_{T}^{\prime}, f_{T}\right)=0, \quad P \in \mathcal{P}(M), r>0,
$$

uniformly for $\sigma^{\prime}$ in the compact set $\widetilde{T}^{\prime}(\mathbb{R})^{X}$ attached to a given $X$ in $\mathfrak{a}_{M} / \mathfrak{a}_{Z}$. Since we are taking the limit of an $\mathfrak{a}_{Z}$-invariant function of $T \in \mathfrak{a}_{M}$, we may as well restrict $T$ to points in our complement $\mathfrak{a}_{M}^{Z}$ of $\mathfrak{a}_{Z}$. We write

$$
\begin{aligned}
& \varepsilon_{M}^{\prime}\left(\phi^{\prime}, X+T, f_{T}\right) \\
& =\left|W\left(M^{\prime}, T^{\prime}\right)\right|^{-1} \int_{\tilde{T}^{\prime}(\mathbb{R})^{X+T}} \eta_{M}^{\prime}\left(\phi^{\prime}, \sigma^{\prime}\right) \varepsilon_{M}^{\prime}\left(\sigma^{\prime}, f_{T}\right) d \sigma^{\prime} \\
& =\left|W\left(M^{\prime}, T^{\prime}\right)\right| \int_{\tilde{T}^{\prime}(\mathbb{R})^{X}} \eta_{M}^{\prime}\left(\phi^{\prime}, \sigma_{T}^{\prime}\right) \varepsilon_{M}^{\prime}\left(\sigma_{T}^{\prime}, f_{T}\right) d \sigma^{\prime} .
\end{aligned}
$$

Since the normalized stable character $\eta_{M}^{\prime}\left(\phi^{\prime}, \sigma_{T}^{\prime}\right)$ is bounded independently of $\sigma_{T}^{\prime}$, we conclude that

$$
\lim _{T, \underset{P, r}{\longrightarrow}}\left(\varepsilon_{M}^{\prime}\left(\phi^{\prime}, X+T, f_{T}\right)\right)=0, \quad X \in \mathfrak{a}_{M} / \mathfrak{a}_{Z}, f \in \mathcal{H}^{\prime}(G, \zeta) .
$$

In order to apply (6.11), we need to examine $\varepsilon_{M}^{\prime}\left(\phi^{\prime}, Y, f_{T}\right)$ as a function of $T$, for any $Y \in \mathfrak{a}_{M} / \mathfrak{a}_{G}$. We shall do so with $f$ in the Schwartz space $\mathcal{C}^{\prime}(G, \zeta)$, and $T$ in the complement $\mathfrak{a}_{M}^{Z}$ of $\mathfrak{a}_{Z}$.

We can obviously write the function (6.10) as a Fourier transform

$$
\varepsilon_{M}^{\prime}\left(\phi^{\prime}, Y, f\right)=\int_{i \mathfrak{a}_{M, Z}^{*}} \varepsilon_{M}^{\prime}\left(\phi_{\lambda}^{\prime}, f\right) e^{-\lambda(Y)} d \lambda, \quad f \in \mathcal{C}^{\prime}(G, \zeta),
$$

on $i \mathfrak{a}_{M, Z}^{*}$. Then

$$
\varepsilon_{M}^{\prime}\left(\phi^{\prime}, Y, f\right)=\sum_{\rho \in \mathcal{R}_{\phi}^{+}} \varepsilon_{M}^{\prime}\left(\phi^{\prime}, \rho, Y, f\right)
$$

where

$$
\varepsilon_{M}^{\prime}\left(\phi^{\prime}, \rho, Y, f\right)=\int_{i \mathfrak{a}_{M, Z}^{*}} \varepsilon_{M}^{\prime}\left(\phi_{\lambda}^{\prime}, \rho_{\lambda}\right) f_{M}\left(\rho_{\lambda}\right) e^{-\lambda(Y)} d \lambda .
$$

The coefficient $\varepsilon_{M}^{\prime}\left(\phi_{\lambda}^{\prime}, \rho_{\lambda}\right)$ is a smooth function of $\lambda$, any derivative of which is slowly increasing. Allowing a minor abuse of notation, we write

$$
\varepsilon_{M}^{\prime}\left(\phi^{\prime}, \rho, H\right), \quad H \in \mathfrak{a}_{M} / \mathfrak{a}_{Z},
$$

for its Fourier transform as a tempered distribution (of rapid decrease) on $\mathfrak{a}_{M} / \mathfrak{a}_{G}$. Since $f_{M}\left(\rho_{\lambda}\right)$ is a Schwartz function of $\lambda$, its Fourier transform

$$
f_{M}(\rho, H)=\int_{i \mathfrak{a}_{M, Z}^{*}} f_{M}\left(\rho_{\lambda}\right) e^{-\lambda(H)} d \lambda, \quad H \in \mathfrak{a}_{M} / \mathfrak{a}_{Z},
$$

is a Schwartz function on $\mathfrak{a}_{M} / \mathfrak{a}_{Z}$. We then write

$$
\varepsilon_{M}^{\prime}\left(\phi^{\prime}, \rho, Y, f\right)=\int_{\mathfrak{a}_{M} / \mathfrak{a}_{Z}} \varepsilon_{M}^{\prime}\left(\phi^{\prime}, \rho, Y-H\right) f_{M}(\rho, H) d H,
$$

where the integral represents the convolution of a tempered distribution with a Schwartz function. It remains to describe $f_{T, M}(\rho, H)$ as a function of $T$.

The Langlands parameter $\phi^{\prime}$ for $\widetilde{M}^{\prime}$ is cuspidal, but its image $\phi$ in $\Phi_{\text {temp }}(M, \zeta)$ of course need not be. We have already accounted implicitly for this possibility in the form (6.9) taken by elements $\rho$ of the basis $\mathcal{F}_{\phi}^{+}$. Consider such a $\rho$. The 
associated virtual character $\tau=\tau_{1}^{M}$ in $T_{\phi}^{+}$is induced from a linear combination of constituents of a representation $\pi_{1}=\pi_{\rho}^{M_{1}}$ of $M_{1}(\mathbb{R})$ induced in turn from a cuspidal representation $\pi_{\rho} \in \Pi_{\text {temp,cusp }}\left(M_{\rho}, \zeta\right)$. The distribution $\rho$ is thus attached to a chain

$$
M_{\rho} \subset M_{1} \subset M
$$

of Levi subgroups of $M$. We can therefore identify the imaginary part $\nu_{\phi}$ of $\chi_{\phi}$ with an imaginary linear form $\nu_{\rho}$ on $\mathfrak{a}_{M_{\rho}}$, which represents the imaginary part of the infinitesimal character of $\rho$. Of course $\nu_{\rho}$ still lies in the subspace $i \mathfrak{a}_{M}^{*}$ of $i \mathfrak{a}_{M_{\rho}}^{*}$, and by the condition we have placed on $\phi^{\prime}$, it vanishes on the subspace $\mathfrak{a}_{M}^{Z}$ that contains $T$. The value of $f_{T, M}$ at any deformation $\tau_{1, \Lambda}^{M}$ of $\tau$ by a point $\Lambda \in i \mathfrak{a}_{M_{1}, Z}^{*}$ is given by a sum over the set $U\left(M, M_{\rho}\right)=U^{G}\left(M, M_{\rho}\right)$ of embeddings of $\mathfrak{a}_{M}$ into $\mathfrak{a}_{M_{\rho}}$. Since the imaginary part of the infinitesimal character $\tau_{1, \Lambda}^{M}$ corresponds to the linear form $\nu_{\rho}+\Lambda$, and

$$
e^{\left(\nu_{\rho}+\Lambda\right)(u T)}=e^{\nu_{\rho}(u T)} e^{\Lambda(u T)}=e^{\Lambda(u T)}, \quad u \in U\left(M, M_{\rho}\right),
$$

we can write

$$
f_{T, M}\left(\tau_{1, \Lambda}^{M}\right)=\sum_{u \in U\left(M, M_{\rho}\right)} e^{\Lambda(u T)} f_{M}\left(\tau_{1, \Lambda}^{M}\right) .
$$

We shall apply this formula, with

$$
\Lambda=\mu+\lambda, \quad \mu \in i \mathfrak{a}_{M_{1}, Z}^{*}, \lambda \in i \mathfrak{a}_{M, Z}^{*},
$$

to compute $f_{T, M}\left(\rho_{\lambda}\right)$.

The differential operator $D$ in (6.9) is defined on $i \mathfrak{a}_{M_{1}}^{*} / i \mathfrak{a}_{M}^{*}$. It acts on functions of $\mu+\lambda$ through the variable $\mu$. It follows from Leibnitz' rule that

$$
\lim _{\mu \rightarrow 0} D\left(e^{(\mu+\lambda)(u T)} f_{M}\left(\tau_{1, \mu+\lambda}^{M}\right)\right)=\sum_{i=1}^{n_{D}} p_{i}(D, u T)\left(\lim _{\mu \rightarrow 0} D_{i} f_{M}\left(\tau_{1, \mu+\lambda}^{M}\right)\right) e^{\lambda(u T)},
$$

for invariant differential operators $\left\{D_{i}\right\}$ on $i \mathfrak{a}_{M_{1}}^{*} / i \mathfrak{a}_{M}^{*}$ and polynomials $\left\{p_{i}(D, \cdot)\right\}$ on $\mathfrak{a}_{M_{1}}^{M}$. The distribution $\rho$ in (6.9) consequently satisfies

$$
f_{T, M}\left(\rho_{\lambda}\right)=\sum_{u \in U\left(M, M_{\rho}\right)}\left(\sum_{i} p_{i}(\rho, u T) f_{M}\left(\rho_{i, \lambda}\right)\right) e^{\lambda(u T)},
$$

where

$$
f_{M}\left(\rho_{i, \lambda}\right)=\lim _{\mu \rightarrow 0} D_{i} f_{M}\left(\tau_{1, \mu+\lambda}^{M}\right)
$$

and

$$
p_{i}(\rho, u T)=p_{i}(D, u T)
$$

We can therefore write

$$
\begin{aligned}
f_{T, M}(\rho, H) & =\int_{i \mathfrak{a}_{M, Z}^{*}} f_{T, M}\left(\rho_{\lambda}\right) e^{-\lambda(H)} d \lambda \\
& =\sum_{u \in U\left(M, M_{\rho}\right)} \sum_{i} p_{i}(\rho, u T) f_{M}\left(\rho_{i}, H-(u T)_{M}\right),
\end{aligned}
$$

where $(u T)_{M}$ is the projection of $u T$ onto $\mathfrak{a}_{M}$. Notice that if $u$ equals the identity embedding 1 of $\mathfrak{a}_{M}$ into $\mathfrak{a}_{M_{\rho}}$, then

$$
D\left(e^{(\mu+\lambda)(u T)} f_{M}\left(\tau_{1, \mu+\lambda}^{M}\right)\right)=e^{(\mu+\lambda)(T)} D f_{M}\left(\tau_{1, \mu+\lambda}^{M}\right) .
$$

In this case, $n_{D}=1, p_{1}(\rho, u T)=1$ and $\rho_{1}=\rho$. 
We conclude that

$$
\begin{aligned}
\varepsilon_{M}^{\prime}\left(\phi^{\prime}, Y, f_{T}\right) & =\sum_{\rho \in R_{\phi}^{+}} \int_{\mathfrak{a}_{M} / \mathfrak{a}_{Z}} \varepsilon_{M}^{\prime}\left(\phi^{\prime}, \rho, Y-H\right) f_{T, M}(\rho, H) d H \\
& =\sum_{\rho} \sum_{u \in U\left(M, M_{\rho}\right)} \sum_{i} p_{i}(\rho, u T) \varepsilon_{i}^{\prime}\left(\phi^{\prime}, \rho, Y-(u T)_{M}, f\right),
\end{aligned}
$$

where

$$
\varepsilon_{i}^{\prime}\left(\phi^{\prime}, \rho, U, f\right)=\int_{\mathfrak{a}_{M} / \mathfrak{a}_{G}} \varepsilon_{M}^{\prime}\left(\phi^{\prime}, \rho, U-H\right) f_{M}\left(\rho_{i}, H\right) d H,
$$

for any $U \in \mathfrak{a}_{M} / \mathfrak{a}_{G}$ and $f \in \mathcal{C}^{\prime}(G, \zeta)$. It is a consequence of the discussion that $\varepsilon_{i}^{\prime}\left(\phi^{\prime}, \rho, U, f\right)$ is a Schwartz function of $U$. If $u=1$, the corresponding inner sum is taken over the one element $i=1$, and reduces simply to $\varepsilon_{M}^{\prime}\left(\phi^{\prime}, \rho, Y-T, f\right)$.

We apply the last expansion to any function $f \in \mathcal{H}^{\prime}(G, \zeta)$. We have established that $\varepsilon_{M}^{\prime}\left(\phi^{\prime}, X+T, f_{T}\right)$ equals

$$
\sum_{\rho \in R_{\phi}^{+}} \sum_{u \in U\left(M, M_{\rho}\right)} \sum_{i} p_{i}(\rho, T) \varepsilon_{i}^{\prime}\left(\phi^{\prime}, \rho, X+T-(u T)_{M}, f\right) .
$$

If $u \neq 1,\left\|T-(u T)_{M}\right\|$ is bounded below by a positive multiple of $\|T\|$, for any $T \in \mathfrak{a}_{P}^{r}$. Since $\varepsilon_{i}^{\prime}\left(\phi^{\prime}, \rho, \cdot, f\right)$ is a rapidly decreasing function on $\mathfrak{a}_{M, Z}$, and $p_{i}(\rho, \cdot)$ is a polynomial on $\mathfrak{a}_{M, Z}$, the summands corresponding to any $u \neq 1$ approach 0 as $T$ approaches infinity in $\mathfrak{a}_{P}^{r}$. If $u=1$, the inner sum over $i$ reduces simply to the function

$$
\varepsilon_{M}^{\prime}\left(\phi^{\prime}, \rho, X+T-(u T)_{M}, f\right)=\varepsilon_{M}^{\prime}\left(\phi^{\prime}, \rho, X, f\right) .
$$

Combining these observations with the limit formula (6.11), we conclude that

$$
\begin{aligned}
\varepsilon_{M}^{\prime}\left(\phi^{\prime}, X, f\right) & =\sum_{\rho \in \mathcal{R}_{\phi}^{+}} \varepsilon_{M}^{\prime}\left(\phi^{\prime}, \rho, X, f\right) \\
& =\lim _{T \underset{P, r}{\longrightarrow}} \varepsilon_{M}^{\prime}\left(\phi^{\prime}, X+T, f_{T}\right)=0
\end{aligned}
$$

for any $X \in \mathfrak{a}_{M, Z}$ and $f \in \mathcal{H}^{\prime}(G, \zeta)$. It then follows from (6.12) that $\varepsilon_{M}^{\prime}\left(\phi_{\lambda}^{\prime}, f\right)=0$ for any $\lambda \in i \mathfrak{a}_{M . Z}^{*}$ and $f \in \mathcal{H}^{\prime}(G, \zeta)$.

We have agreed that $\varepsilon_{M}^{\prime}\left(\phi_{\lambda}^{\prime}, f\right)$ is supported on characters. In other words, it descends to a continuous linear form

$$
\widehat{\varepsilon}_{M}^{\prime}\left(\phi_{\lambda}^{\prime}, f_{G}\right)=\varepsilon_{M}^{\prime}\left(\phi_{\lambda}^{\prime}, f\right), \quad f \in \mathcal{C}^{\prime}(G, \zeta),
$$

on the image $\mathcal{I}^{\prime}(G, \zeta)$ of $\mathcal{C}^{\prime}(G, \zeta)$ in $\mathcal{I}(G, \zeta)$. From what we have just seen, $\widehat{\varepsilon}_{M}^{\prime}\left(\phi_{\lambda}^{\prime}, \cdot\right)$ vanishes on the subspace

$$
I \mathcal{H}^{\prime}(G, \zeta)=I \mathcal{H}(G, \zeta) \cap \mathcal{I}^{\prime}(G, \zeta)
$$

of $\mathcal{I}^{\prime}(G, \zeta)$. But by the two versions $\mathrm{CD}$ and $\mathrm{A} 8$, of the trace Paley-Wiener theorem, $I \mathcal{H}^{\prime}(G, \zeta)$ is dense in $\mathcal{I}^{\prime}(G, \zeta)$. We conclude that $\varepsilon_{M}^{\prime}\left(\phi_{\lambda}^{\prime}, f\right)$ vanishes for any $f \in \mathcal{C}^{\prime}(G, \zeta)$. This is what we had to prove. As we have seen, it implies that $\varepsilon_{M}^{\prime}\left(\sigma^{\prime}, f\right)$ vanishes for any $\sigma^{\prime} \in \widetilde{T}^{\prime}(\mathbb{R})$, the uniform statement to which we have reduced all the assertions of Theorem 1.1. Our proof of Theorem 1.1 is at last complete.

We close with a couple of comments. For fixed $f$, the objects of Theorem 1.1 belong to the space

$$
C_{G-\mathrm{reg}}^{\infty}\left(T^{\prime}, M, \zeta\right)=C^{\infty}\left(T_{G-\mathrm{reg}}^{\prime}, M, \zeta\right)
$$


of smooth sections of a line bundle over $\bar{T}_{G \text {-reg }}^{\prime}(\mathbb{R})$. We have been treating the variable $\sigma^{\prime}$ as a representative in $\widetilde{T}_{G \text {-reg }}^{\prime}(\mathbb{R})$ of a point in the base space

$$
\bar{T}_{G \text {-reg }}^{\prime}(\mathbb{R})=T_{G \text {-reg }}^{\prime}(\mathbb{R}) / Z(\mathbb{R})=\widetilde{T}_{G \text {-reg }}^{\prime}(\mathbb{R}) / \widetilde{Z}^{\prime}(\mathbb{R}) .
$$

The associated functions of Theorem 1.1 depend only on the stable conjugacy class $\delta^{\prime}$ of $\sigma^{\prime}$ in $\widetilde{M}^{\prime}(\mathbb{R})$. They can in fact be regarded as sections of a line bundle that depends only on the isomorphism class of $M^{\prime}$.

Let us change notation slightly. We write $\delta^{\prime}$ in place of $\sigma^{\prime}$, and we let $M^{\prime}$ denote simply an isomorphism class of elliptic endoscopic data for $M$. We still take $\mathcal{T}\left(M^{\prime}, M\right)$ to be the associated set of transfer factors $\Delta=\Delta_{M}$, with the understanding that any $\Delta$ now includes an implicit choice of representative within the isomorphism class $M^{\prime}$, as well as an auxiliary datum $\left(\widetilde{M}^{\prime}, \widetilde{\xi}^{\prime}\right)$ for that representative. We can then define a bundle $\mathcal{L}_{G \text {-reg,ell }}\left(M^{\prime}, M, \zeta\right)$, consisting of the set of equivalence classes of pairs

$$
\left(\Delta, \delta^{\prime}\right), \quad \Delta \in \mathcal{T}\left(M, M^{\prime}\right), \delta^{\prime} \in \widetilde{M}_{G \text {-reg,ell }}^{\prime}(\mathbb{R}) .
$$

The prescription is similar to that of $\S 1$, except for the fact that it has an extra condition of equivalence, corresponding to isomorphisms of endoscopic data. Then $\mathcal{L}_{G \text {-reg,ell }}\left(M^{\prime}, M, \zeta\right)$ becomes a principal $U(1)$-bundle over the space $\Delta_{G \text {-reg,ell }}\left(M^{\prime}, Z\right)$ of isomorphism classes of pairs $\left(\bar{M}^{\prime}, \bar{\delta}^{\prime}\right)$, where $\bar{M}^{\prime}$ is the quotient by $Z$ of a representative within the class $M^{\prime}$, and $\bar{\delta}^{\prime}$ is a strongly $G$-regular, elliptic element in $\bar{M}^{\prime}(\mathbb{R})$. We set $C_{G \text {-reg,ell }}^{\infty}\left(M^{\prime}, M, \zeta\right)$ equal to the space of smooth sections of the line bundle dual to $\mathcal{L}_{G \text {-reg,ell }}\left(M^{\prime}, M, \zeta\right)$. One can then show that for fixed $f$, the objects $I_{M}\left(\delta^{\prime}, f\right), I_{M}^{\mathcal{E}}\left(\delta^{\prime}, f\right)$ and $S_{M}^{G}\left(M^{\prime}, \delta^{\prime}, f\right)$ of Theorem 1.1 belong to $C_{G \text {-reg,ell }}^{\infty}\left(M^{\prime}, M, \zeta\right)$. We refer the reader to forthcoming papers [A15, §1-2] and [A16, §4], where these notions are treated in greater generality.

Suppose that $\gamma$ is a strongly $G$-regular, elliptic conjugacy class in $M(\mathbb{R})$. If $\Delta_{M}$ belongs to $\mathcal{T}\left(M^{\prime}, M\right)$, the function

$$
\delta^{\prime} \longrightarrow \Delta_{M, \zeta}\left(\delta^{\prime}, \gamma\right)=\sum_{z \in Z(\mathbb{R})} \Delta_{M}\left(\delta^{\prime}, z \gamma\right) \zeta(z)^{-1}
$$

can be regarded as a section of $\mathcal{L}_{G \text {-reg,ell }}\left(M^{\prime}, M, \zeta\right)$ of finite support. Set

$$
\Delta_{M, \zeta}\left(\gamma, \delta^{\prime}\right)=n_{\gamma}^{-1} \overline{\Delta_{M, \zeta}\left(\delta^{\prime}, \gamma\right)}
$$

where $n_{\gamma}$ is the number of $M(\mathbb{R})$-conjugacy classes in the stable class of $\gamma$. If $\gamma_{1}$ is another strongly $G$-regular, elliptic class in $M(\mathbb{R})$, the sum

$$
\sum_{M^{\prime}} \sum_{\delta^{\prime} \in \Delta_{G-\mathrm{reg}}\left(\bar{M}^{\prime}\right)} \Delta_{M, \zeta}\left(\gamma, \delta^{\prime}\right) \Delta_{M, \zeta}\left(\delta^{\prime}, \gamma_{1}\right)
$$

vanishes unless $\gamma_{1}=\gamma z$, for some element $z \in Z(\mathbb{R})$, in which case it equals $\zeta(z)$. This relation follows from A11, Lemma 2.3]. It provides an inversion formula

$$
I_{M}(\gamma, f)=\sum_{M^{\prime}} \sum_{\delta^{\prime} \in \Delta_{G^{-r e g}}\left(\bar{M}^{\prime}\right)} \Delta_{M, \zeta}\left(\gamma, \delta^{\prime}\right) I_{M}\left(\delta^{\prime}, f\right)
$$

for the function (1.4) that is the source of Theorem 1.1. Set

$$
I_{M}^{\mathcal{E}}(\gamma, f)=\sum_{M^{\prime}} \sum_{\delta^{\prime} \in \Delta_{G^{- \text {reg }}}\left(\bar{M}^{\prime}\right)} \Delta_{M, \zeta}\left(\gamma, \delta^{\prime}\right) I_{M}^{\mathcal{E}}\left(\delta^{\prime}, f\right) .
$$


Statement (a) of Theorem 1.1 is then equivalent to the identity

$$
I_{M}^{\mathcal{E}}(\gamma, f)=I_{M}(\gamma, f) \text {. }
$$

Statement (b) of Theorem 1.1 is of course unchanged if $\sigma^{\prime}$ is replaced by $\delta^{\prime}$. It is in this form that Theorem 1.1 was conjectured [A11, Conjecture 3.3], and later applied as [A13, Local Theorem 1 (p. 775)].

\section{ACKNOWLEDGMENT}

I am indebted to the referee(s) for a close reading of the paper and for very helpful suggestions.

\section{REFERENCES}

[A1] J. Arthur, Harmonic analysis of the Schwartz space on a reductive Lie group, Parts I and II, mimeographed notes.

[A2] - The characters of discrete series as orbital integrals, Invent. Math. 32 (1976), 205-261. MR0412348(54:474)

[A3] _ The local behaviour of weighted orbital integrals, Duke Math. J. 56 (1988), 223-293. MR932848 (89h:22036)

[A4] The invariant trace formula I. Local theory, J. Amer. Math. Soc. 1 (1988), 323-383. MR928262 (89e:22029)

[A5] , Intertwining operators and residues I. Weighted characters, J. Funct. Anal. 84 (1989), 19-84. MR999488 (90j:22018)

[A6] - On elliptic tempered characters, Acta Math. 171 (1993), 73-138. MR1237898 (94i:22038)

[A7] On the Fourier transforms of weighted orbital integrals, J. Reine Agnew. Math. 452 (1994), 163-217. MR 1282200 (95h:22015)

[A8] - The trace Paley-Wiener theorem for Schwartz functions, Contemp. Math. 177 (1994), 171-180. MR1303605 (95k:22010)

[A9] Canonical normalization of weighted characters and a transfer conjecture, C.R. Math. Acad. Sci. Soc. R. Can. 20 (2), (1998), 33-52. MR1623485 (99g:22020)

[A10] _ Endoscopic L-functions and a combinatorial identity, Canad. J. Math. 51 (1999), 1135-1148. MR 1756875 (2001g:11071)

[A11] _ On the transfer of distributions: weighted orbital integrals, Duke Math. J. 99 (1999), 209-283. MR1708030 (2000i:22023)

[A12] - Stabilization of a family of differential equations, Proc. Sympos. Pure Math., vol. 68, 2000, 77-95. MR:1767893(2001f:22025)

[A13] _ A stable trace formula III. Proof of the main theorems, Annals of Math. 158 (2003), 769-873. MR2031854 (2004m:11079)

[A14] _ An asymptotic formula for real groups, J. Reine Angew. Math. 601 (2006), 163230. MR2289209

[A15] _ Singular invariant distributions and endoscopy, in preparation.

[A16] - On the transfer of distributions: singular orbital integrals, in preparation.

[AC] J. Arthur and L. Clozel, Simple Algebras, Base Change, and the Advanced Theory of the Trace Formula, Ann. of Math. Studies 120, Princeton Univ. Press, Princeton, N.J., 1989. MR1007299 (90m:22041)

[CD] L. Clozel and P. Delorme, Le théorème de Paley-Wiener invariant pour les groupes de Lie réductifs II, Ann. Scient. Éc. Norm. Sup. (4), 23 (1990), 193-228. MR1046496 (91g:22013)

[H1] Harish-Chandra, Invariant eigendistributions on a semisimple Lie group, Trans. Amer. Math. Soc. 119, 457-508. MR0180631 (31:4862d)

[H2] - Harmonic analysis on real reductive groups, I. The theory of the constant term, J. Funct. Anal. 19, 104-204. MR0399356 (53:3201)

[K1] R. Kottwitz, Rational conjugacy classes in reductive groups, Duke Math. J. 49 (1982), 785-806. MR683003 (84k:20020)

[K2] Stable trace formula: elliptic singular terms, Math. Ann. 275 (1986), 365-399. MR858284 (88d:22027) 
[KS] R. Kottwitz and D. Shelstad, Foundations of Twisted Endoscopy, Astérisque, vol. 255. MR.1687096 (2000k:22024)

[L1] R. Langlands, Stable conjugacy: definitions and lemmas, Canad. J. Math. 31 (1979), 700725. MR540901 (82j:10054)

[L2] Cancellation of singularities at real places, notes from a lecture, Institute for Advanced Study, Princeton, N.J., 1984.

[L3] - On the classification of irreducible representations of real algebraic groups, in Representation Theory and Harmonic Analysis on Semisimple Lie Groups, AMS Mathematical Surveys and Monographs, vol. 31, 1989, 101-170. MR1011897(91e:22017)

[L4] Beyond endoscopy, in Contributions to Automorphic Forms, Geometry, and Number Theory, Johns Hopkins University Press, 2004, 611-698. MR2058622 (2005f:11102)

[L5] Un nouveau point de repère dans la théorie des formes automorphes, to appear in Canad. Math. Bull.

[LS1] R. Langlands and D. Shelstad, On the definition of transfer factors, Math. Ann. 278 (1987), 219-271. MR909227 (89c:11172)

[LS2] — Descent for transfer factors, The Grothendieck Festschift, Vol II, Birkhauser, Boston, 1990, 485-563. MR 1106907 (92i:22016)

[S1] D. Shelstad, Characters and inner forms of a quasisplit group over $\mathbb{R}$, Compositio Math. 39 (1979), 11-45. MR539000 (80m:22023)

[S2] - Orbital integrals and a family of groups attached to a real reductive group, Ann. Scient. Éc. Norm. Sup. 12, (1979), 1-31. MR532374 (81k:22014)

[S3] _ L-indistinguishability for real groups, Math. Ann. 259 (1982), 385-430. MR661206 (84c:22017)

Department of Mathematics, University of Toronto, Bahen Centre, 6th Floor, 40 St George Street, Toronto, ON M5S 2E4 Canada 